\title{
Isomorphism of regular trees and words
}

\author{
Markus Lohrey and Christian Mathissen \\ Institut für Informatik, Universität Leipzig, Germany \\ \{lohrey, mathissen\}@informatik.uni-leipzig.de
}

\begin{abstract}
The computational complexity of the isomorphism problem for regular trees, regular linear orders, and regular words is analyzed. A tree is regular if it is isomorphic to the prefix order on a regular language. In case regular languages are represented by NFAs (DFAs), the isomorphism problem for regular trees turns out to be EXPTIME-complete (resp. P-complete). In case the input automata are acyclic NFAs (acyclic DFAs), the corresponding trees are (succinctly represented) finite trees, and the isomorphism problem turns out to be PSPACEcomplete (resp. P-complete). A linear order is regular if it is isomorphic to the lexicographic order on a regular language. A polynomial time algorithm for the isomorphism problem for regular linear orders (and even regular words, which generalize the latter) given by DFAs is presented. This solves an open problem by Ésik and Bloom.
\end{abstract}

\section{Introduction}

Isomorphism problems for infinite but finitely presented structures are an active research topic in algorithmic model theory [1]. It is a folklore result in computable model theory that the isomorphism problem for computable structures (i.e., structures, where the domain is a computable set of natural numbers and all relations are computable too) is highly undecidable - more precisely, it is $\Sigma_{1}^{1}$-complete, i.e., complete for the first existential level of the analytical hierarchy. Khoussainov et al. proved in [17] that even for automatic structures (i.e., structures, where the domain is a regular set of words and all relations can be recognized by synchronous multitape automata), the isomorphism problem is $\Sigma_{1}^{1}$-complete. In [19], this result was further improved to automatic order trees and automatic linear orders. On the decidability side, Courcelle proved that the isomorphism problem for equational graphs is decidable [7]. Recall that a graph is equational if it is the least solution of a system of equations over the HR graph operations. We remark that Courcelle's algorithm for the isomorphism problem for equational graphs has very high complexity (it is not elementary), since it uses the decidability of monadic second-order logic on equational graphs.

In this paper, we continue the investigation of isomorphism problems for infinite but finitely presented structures at the lower end of the spectra. We focus on two very simple classes of infinite structures: regular trees and regular words. Both are particular automatic structures. Recall that a countable tree is regular if it has only finitely many subtrees up to isomorphism. This definition works for ordered trees (where the children of a node are linearly ordered) and unordered trees. An equivalent characterization in the unordered case uses regular languages: An unordered (countable) tree $T$ is regular 
if and only if there is a regular language $L \subseteq \Sigma^{*}$ which contains the empty word and such that $T$ is isomorphic to the tree obtained by taking the prefix order on $L$ (the empty word word is the root of the tree). Hence, a regular tree can be represented by a finite deterministic or nondeterministic automaton (DFA or NFA), and the isomorphism problem for regular trees becomes the following computational problem: Given two DFAs (resp., NFAs) accepting both the empty word, are the corresponding regular trees isomorphic? It is is not difficult to prove that this problem can be solved in polynomial time if the two input automata are assumed to be DFAs; the algorithm is very similar to the well-known partition refinement algorithm for checking bisimilarity of finite state systems [15], see Section 3.1. Hence, the isomorphism problem for regular trees that are represented by NFAs can be solved in exponential time. Our first main result states that this problem is in fact EXPTIME-complete, see Section 3.2. The proof of the EXPTIME lower bound uses three main ingredients: (i) EXPTIME coincides with alternating polynomial space [5], (ii) a construction from [14], which reduces the evaluation problem for Boolean expressions to the isomorphism problem for (finite) trees, and (iii) a small NFA accepting all words that do not represent an accepting computation of a polynomial space machine [28]. ${ }^{1}$. Our proof technique yields another result too: It is PSPACE-complete to check for two given acyclic NFAs $\mathcal{A}_{1}, \mathcal{A}_{2}$ (both accepting the empty word), whether the trees that result from the prefix orders on $L\left(\mathcal{A}_{1}\right)$ and $L\left(\mathcal{A}_{2}\right)$, respectively, are isomorphic. Note that these two trees are clearly finite (since the automata are acyclic), but the size of $L\left(\mathcal{A}_{i}\right)$ can be exponential in the number of states of $\mathcal{A}_{i}$. In this sense, acyclic NFAs can be seen as a succinct representation of finite trees. The PSPACE-upper bound for acyclic NFAs follows easily from Lindell's result [21] that isomorphism of explicitly given trees can be checked in logarithmic space.

The second part of this paper studies the isomorphism problem for regular words, which were introduced in [6]. A generalized word over an alphabet $\Sigma$ is a countable linear order together with a $\Sigma$-coloring of the elements. A generalized word is regular if it can be obtained as the least solution (in a certain sense made precise in [6]) of a system $X_{1}=t_{1}, \ldots, X_{n}=t_{n}$. Here, every $t_{i}$ is a finite word over the alphabet $\Sigma \cup\left\{X_{1}, \ldots, X_{n}\right\}$. For instance, the system $X=a b X$ defines the regular word $(a b)^{\omega}$. Courcelle [6] gave an alternative characterization of regular words: A generalized word is regular if and only if it is equal to the frontier word of a finitely-branching ordered regular tree, where the leaves are colored by symbols from $\Sigma$. Here, the frontier word is obtained by ordering the leaves in the usual left-to-right order (note that the tree is ordered). Alternatively, a regular word can be represented by a DFA $\mathcal{A}$, where the set of final states is partitioned into sets $F_{a}(a \in \Sigma)$; we call such a DFA a partitioned DFA. The corresponding regular word is obtained by ordering the language of $\mathcal{A}$ lexicographical and coloring a word $w \in L(\mathcal{A})$ with $a$ if $w$ leads from the initial state to a state from $F_{a}$. A third characterization of regular words was provided by Heilbrunner [13]: A generalized word is regular if it can be obtained from singleton words (i.e., symbols from $\Sigma$ ) using the operations of concatenation, $\omega$-power, $\bar{\omega}$-power and dense shuffle. For a generalized word $u$, its $\omega$-power (resp. $\bar{\omega}$-power) is the generalized word uuu ... (resp. $\cdots u u u$ ). Moreover, the shuffle of generalized words $u_{1}, \ldots, u_{n}$ is obtained by

\footnotetext{
${ }^{1}$ This construction is used in [28] to prove that the universality problem for NFAs is PSPACEcomplete.
} 
choosing a dense coloring of the rationals with colors $\{1, \ldots, n\}$ (up to isomorphism, there is only a single such coloring [26]) and then replacing every $i$-colored rational by $u_{i}$. In fact, Heilbrunner presents an algorithm which computes from a given system of equations (or, alternatively, a partitioned DFA) an expression over the above set of operations (called a regular expression in the following) which defines the least solution of the system of equations. A simple analysis of Heilbrunner's algorithm shows that the computed regular expression in general has exponential size with respect to the input system of equations and it is easy to see that this cannot be avoided. ${ }^{2}$ The next step was taken by Thomas in [29], where he proved that the isomorphism problem for regular words is decidable. For his proof, he uses the decidability of the monadic second-order theory of linear orders; hence his proof does not yield an elementary upper bound for the isomorphism problem for regular words. Such an algorithm was presented later by Bloom and Ésik in [2], where the authors present a polynomial time algorithm for checking whether two given regular expressions define isomorphic regular words. Together with Heilbrunner's algorithm, this yields an exponential time algorithm for checking whether the least solutions of two given systems of equations (or, alternatively, the regular words defined by two partitioned DFAs) are isomorphic. It was asked in [2], whether a polynomial time algorithm for this problem exists. Our second main result answers this question affirmatively. In fact, we prove that the problem, whether two given partitioned DFAs define isomorphic regular words, is P-complete. A large part of this paper deals with the polynomial time upper bound. The first step is simple. By reanalyzing Heilbrunner's algorithm, it is easily seen that from a given partitioned DFA (defining a regular word $u$ ) one can compute in polynomial time a succinct representation of a regular expression for $u$. This succinct representation consists of a DAG (directed acyclic graph), whose unfolding is a regular expression for $u$. The second and main step of the proof shows that the polynomial time algorithm of Bloom and Ésik for regular expressions can be refined in such a way that it works (in polynomial time) for succinct regular expressions too. The main tool in our proof is (besides the machinery from [2]) algorithmics on compressed strings (see [27] for a survey), in particular Plandowski's result that equality of strings that are represented by straight-line programs (i.e., context free grammars that only generate a single word) can be checked in polynomial time [24]. It is a simple observation that an acyclic partitioned DFA is basically a straight-line program. Hence, we show how to extend Plandowski's polynomial time algorithm from acyclic partitioned DFAs to general partitioned DFAs.

An immediate corollary of our result is that it can be checked in polynomial time whether the lexicographic orderings on the languages defined by two given DFAs (so called regular linear orderings) are isomorphic. For the special case that the two input DFAs accept well-ordered languages, this was shown in [8]. Let us mention that it is highly undecidable ( $\Sigma_{1}^{1}$-complete) to check, whether the lexicographic orderings on the languages defined by two given deterministic pushdown automata (these are the algebraic linear orderings [3]) are isomorphic [19].

\footnotetext{
${ }^{2}$ Take for instance the system $X_{i}=X_{i+1} X_{i+1}(1 \leq i \leq n), X_{n}=a$, which defines the finite word $a^{2^{n}}$.
} 


\section{Preliminaries}

For an equivalence relation $R$ on a set $A$ and $a \in A$ we denote with $[a]_{R}$ the equivalence class containing $R$. Moreover, $[A]_{R}=\left\{[a]_{R} \mid a \in A\right\}$. Let us take a finite alphabet $\Sigma$. The length of a finite words $u \in \Sigma^{*}$ is denoted by $|u|$. Let $\Sigma^{+}=\left\{u \in \Sigma^{*}|| u \mid>0\right\}$, $\Sigma^{k}=\left\{u \in \Sigma^{*}|| u \mid=k\right\}, \Sigma^{\leq k}=\left\{u \in \Sigma^{*}|| u \mid \leq k\right\}$, and $\Sigma^{\geq k}=\left\{u \in \Sigma^{*}|| u \mid \geq\right.$ $k\}$. For $u, v \in \Sigma^{*}$, we write $u \leq_{\text {pref }} v$ if there exists $w \in \Sigma^{*}$ with $v=u w$, i.e., $u$ is a prefix of $v$. We write $u<_{\text {pref }} v$ if $u \leq_{\text {pref }} v$ and $u \neq v$. For a language $L \subseteq \Sigma^{*}$ let $\operatorname{pref}(L)=\left\{u \in \Sigma^{*} \mid \exists v \in L: u \leq\right.$ pref $\left.v\right\}$. For a fixed linear order $\leq$ on the alphabet $\Sigma$ we define the lexicographic order $\leq_{\text {lex }}$ on $\Sigma^{*}$ as follows: $u \leq_{\operatorname{lex}} v$ if $u \leq_{\text {pref }} v$ or there exist words $w, x, y$ and $a, b \in \Sigma$ such that $a<b, u=w a x$, and $v=w b y$.

\subsection{Complexity theory}

We assume that the reader has some basic background in complexity theory, in particular concerning the complexity classes NL, P, PSPACE, and EXPTIME, see e.g. [23]. All completeness results in this paper refer to logspace reductions.

A PSPACE-transducer is a deterministic Turing machine with a read-only input tape, a write-only output tape and a work tape, whose length is bounded by $n^{O(1)}$, where $n$ is the input length. The output is written from left to right on the output tape, i.e., in each step the transducer either outputs a new symbol on the output tape, in which case the output head moves one cell to the right, or the transducer does not output a new symbol in which case the output head does not move. Moreover, we assume that the transducer terminates for every input. This implies that a PSPACE-transducer computes a mapping $f: \Sigma^{*} \rightarrow \Theta^{*}$, where $|f(w)|$ is bounded by $2^{|w|^{O(1)}}$. We need the following simple lemma:

Lemma 2.1. Assume that the mapping $f: \Sigma^{*} \rightarrow \Theta^{*}$ can be computed by a PSPACEtransducer and let $L \subseteq \Theta^{*}$ be a language in $\operatorname{NSPACE}\left(\log ^{k}(n)\right)$ for some constant $k$. Then $f^{-1}(L)$ belongs to PSPACE.

Proof. The proof uses the same idea that shows that the composition of two logspace computable mappings is again logspace computable. Let $w \in \Sigma^{*}$ be an input. Basically, we run the $\operatorname{NSPACE}\left(\log ^{k}(n)\right)$-algorithm for $L$ on the input $f(w)$. But since $f$ can be computed by a PSPACE-transducer (which can generate an exponentially long output) the length of $f(w)$ can be only bounded by $2^{|w|^{O(1)}}$. Hence, we cannot construct $f(w)$ explicitly. But this is not necessary. We only store a pointer to some position $f(w)$ (this pointer needs space $|w|^{O(1)}$ ) while running the NSPACE $\left(\log ^{k}(n)\right)$-algorithm for $L$. Each time, this algorithm needs the $i^{\text {th }}$ letter of $f(w)$, we run the PSPACE-transducer for $L$ until the $i^{t h}$ output symbol is generated. The first $i-1$ symbols of $f(w)$ are not written on the output tape. Note that the NSPACE $\left(\log ^{k}(n)\right)$-algorithm for $L$ needs space $\log ^{k}\left(2^{|w|^{O(1)}}\right)=|w|^{O(1)}$ while running on $f(w)$. Hence, the total space requirement is bounded by $|w|^{O(1)}$.

An alternating Turing machine is an ordinary nondeterministic Turing machine, where in addition the set of states $Q$ is partitioned into existential states $\left(Q_{\exists}\right)$ and universal 
states $\left(Q_{\forall}\right)$. A configuration, where the current state is existential (resp., universal) is called an existential (resp., universal) configuration. Let us assume that $M$ is an alternating Turing machine without infinite computation paths. Then, we define inductively the notion of an accepting configuration as follows: If $c$ is an existential configuration, then $c$ is accepting if and only if $c$ has an accepting successor configuration. If $c$ is a universal configuration, then $c$ is accepting if and only if all successor configurations of $c$ are accepting. Note that a universal configuration without successor configurations is accepting, whereas an existential configuration without successor configurations is not accepting. An input $x$ is accepted by $M$ (briefly, $x \in L(M)$ ) if and only if the initial configuration with input $x$ is accepting.

The complexity class $\mathrm{C}_{=} \mathrm{P}$ consists of all languages $L \subseteq \Sigma^{*}$ such that there exist nondeterministic polynomial time Turing machines $M_{1}$ and $M_{2}$ with input alphabet $\Sigma$ such that for every input $w \in \Sigma^{*}: w \in L$ if and only if the number of accepting computations of $M_{1}$ on input $w$ equals the number of accepting computations of $M_{2}$ on input $w$. If we replace in this definition nondeterministic polynomial time Turing machines by nondeterministic $\operatorname{logspace}$ Turing machines, we obtain the class $C_{=} L$.

\subsection{Finite automata and transducer}

Let $\mathcal{A}=\left(Q, \Sigma, \delta, q_{0}, F\right)$ be a nondeterministic finite automaton, briefly $N F A$, where $Q$ is the set of states, $\Sigma$ is the input alphabet, $\delta \subseteq Q \times \Sigma \times Q$ is the transition relation, $q_{0} \in Q$ is the initial state, and $F \subseteq Q$ is the set of final states. A state $q \in Q$ is accessible (resp. coaccessible), if $q$ can be reached from the initial state $q_{0}$ (resp., if a final state from $F$ can be reached from $q$ ). We say that $\mathcal{A}$ is accessible (resp., coaccessible), if every state of $\mathcal{A}$ is accessible (resp, coaccessible). An NFA $\mathcal{A}$ is called prefix-closed if every state of $\mathcal{A}$ is a final state. In that case, the language $L(\mathcal{A})$ is prefix-closed. Moreover, if $\mathcal{A}$ is coaccessible and the prefix-closed NFA $\mathcal{B}$ results from $\mathcal{A}$ by making every state final, then clearly $L(\mathcal{B})=\operatorname{pref}(L(\mathcal{A})$ ). For a DFA (deterministic finite automaton), $\delta$ is a partial map from $Q \times \Sigma$ to $Q$. Sometimes, we will also deal with NFAs (DFAs) without an initial state. If $\mathcal{A}$ is an NFA without an initial state and $q$ is a state of $\mathcal{A}$, then $L(\mathcal{A}, q)$ is the language accepted by $\mathcal{A}$, when $q$ is declared to be the initial state. We will need the following simple lemma, which is probably folklore:

Lemma 2.2. For a given a DFA $\mathcal{A}=\left(Q, \Sigma, \delta, q_{0}, F\right)$, we can compute the cardinality $|L(\mathcal{A})| \in \mathbb{N} \cup\{\infty\}$ in polynomial time.

Proof. W.l.o.g we can assume that $\mathcal{A}$ is accessible and coaccessible. Then $L(\mathcal{A})$ is finite if and only if $\mathcal{A}$ is acyclic. So assume that $\mathcal{A}$ is acyclic. Since $\mathcal{A}$ is deterministic, the size of $L(\mathcal{A})$ equals the number of paths from $q_{0}$ to $F$. Now, in a directed acyclic graph, the number of paths from a source node to all other nodes can be easily computed by dynamic programming in polynomial time.

A partitioned DFA is a tuple $\mathcal{A}=\left(Q, \Sigma, \delta, q_{0},\left(F_{a}\right)_{a \in \Gamma}\right)$, where $\Gamma$ is a finite alphabet, $\mathcal{B}=\left(Q, \Sigma, \delta, q_{0}, \bigcup_{a \in \Gamma} F_{a}\right)$ is an ordinary DFA and $F_{a} \cap F_{b}=\emptyset$ for $a \neq b$. Since $\mathcal{B}$ is a DFA, it follows that the language $L(\mathcal{B})$ is partitioned by the languages $L\left(\mathcal{A}_{a}\right)$, where $\mathcal{A}_{a}=\left(Q, \Sigma, \delta, q_{0}, F_{a}\right)(a \in \Gamma)$. We use partitioned DFAs to label elements of a structure with symbols from $\Gamma$. The language $L\left(\mathcal{A}_{a}\right)$ will be the set of $a$-labelled 
elements. We do not introduce partitioned NFAs, since for NFAs the languages $L\left(\mathcal{A}_{a}\right)$ $(a \in \Gamma$ ) would not partition $L(\mathcal{B})$ (thus, a point could get several labels).

A ( $\varepsilon$-free) rational transducer is a tuple $\mathcal{T}=\left(Q, \Sigma, \Gamma, \delta, q_{0}, F\right)$, where $Q$ (the set of states), $\Sigma$ (the input alphabet), and $\Gamma$ (the output alphabet) are finite sets, $q_{0} \in Q$ is the initial state, $F \subseteq Q$ is the set of final states, and $\delta \subseteq Q \times \Sigma \times \Gamma^{+} \times Q$ is the transition relation. A transition $(q, a, w, p) \in \delta$ is also written as $q \stackrel{a \mid w}{\longrightarrow} p$. The rational transducer $\mathcal{T}$ defines a binary relation $\llbracket \mathcal{T} \rrbracket \subseteq \Sigma^{*} \times \Gamma^{*}$ in the usual way. For a language $L \subseteq \Sigma^{*}$ let $\mathcal{T}(L)=\left\{v \in \Gamma^{*} \mid \exists u \in L:(u, v) \in \llbracket \mathcal{T} \rrbracket\right\}$.

\subsection{Trees}

A tree is a partial order $T=(A ; \leq)$, where $\leq$ has a smallest element (the root of the tree; in particular $A \neq \emptyset$ ) and for every $a \in A$, the set $\{b \in A \mid b \leq a\}$ is finite and linearly ordered by $\leq$. We write $a \lessdot b$ if $a<b$ and there does not exist $c \in A$ with $a<c<b$. For $a \in A$, let child $(a, T)$ (the set of children of $a$ ) be the set of all $b \in A$ such that $a \lessdot b$. The set of leaves of $T$ is $\operatorname{leaf}(T)=\{a \in A \mid \operatorname{child}(a, T)=\emptyset\}$. For $a \in A$ let $T \uparrow_{a}$ be the subtree of $T$ rooted at $a$, i.e., the set of nodes of $T \uparrow_{a}$ is $\{b \in A \mid a \leq b\}$. The tree $T$ is finitely branching if child $(a, T)$ is finite for all $a \in A$. An infinite path of $T$ is an infinite chain $a_{0} \lessdot a_{1} \lessdot a_{2} \lessdot \cdots$; finite paths are defined analogously. If $T$ is finite and $a \in A$, then the height of $a$ in $T$ is the maximal length of a path that starts in $a$. For trees $T_{1}$ and $T_{2}$ we write $T_{1} \cong T_{2}$ in case $T_{1}$ and $T_{2}$ are isomorphic.

A tree over the finite alphabet $\Sigma$ is a pair $T=\left(L ; \leq_{\text {pref }}\right)$, where $L \subseteq \Sigma^{*}$ is a language with $\varepsilon \in L$. Note that $T$ is indeed a tree in the above sense. Most of the time, we will identify the language $L$ with the tree $\left(L ; \leq_{\text {pref }}\right)$. Moreover, if $L=\operatorname{pref}(L)$ (i.e., $L$ is prefix-closed), then $T$ is a finitely branching tree.

A countable tree $T$ is called regular if $T$ has only finitely many subtrees up to isomorphism. Equivalently, a countable tree is regular if it is isomorphic to a tree of the form $\left(L ; \leq_{\text {pref }}\right)$, where $L$ is a regular language with $\varepsilon \in L$. We require that the empty word $\varepsilon$ belongs to $L$ in order to ensure the existence of a root (otherwiese $(L ; \leq$ pref $)$ would be only a forest). If $L$ is accepted by the accessible DFA $\mathcal{A}$, then the subtrees of $\left(L ; \leq_{\text {pref }}\right)$ correspond to the final states of $\mathcal{A}$. Note that by our definition, a regular tree need not be finitely branching.

Our definition of a regular tree (having only finitely many subtrees up to isomorphism) makes sense for other types of trees as well, e.g. for node-labeled trees or ordered trees (where the children of a node are linearly ordered). These variants of regular trees can be generated by finite automata as well. For instance, a node-labeled regular tree $\left(L ; \leq_{\text {pref }},\left(L_{a}\right)_{a \in \Gamma}\right)$, where $\Gamma$ is the finite labeling alphabet and $L_{a}$ is the set of $a$-labeled nodes can be specified by a partitioned DFA $\left(Q, \Sigma, \delta, q_{0},\left(F_{a}\right)_{a \in \Gamma}\right)$ with $L_{a}=L\left(Q, \Sigma, \delta, q_{0}, F_{a}\right)$ and $L=\bigcup_{a \in \Gamma} L_{a}$. We do not consider node labels in this paper, since it makes no difference for the isomorphism problem (node labels can be eliminated by adding additional children to nodes). Ordered regular trees will be briefly considered in Section 4.8. 


\subsection{Linear orders}

See [26] for a thorough introduction into linear orders. Let $\eta$ be the order type of the rational numbers, $\omega$ the order type of the natural number, and $\bar{\omega}$ be the order type of the negative integers. With $\mathbf{n}$ we denote a finite linear order with $n$ elements. Let $\Lambda=$ $(L ; \leq)$ be a linear order. $\Lambda$ is dense if $L$ consists of at least two elements, and for all $x<y$ there exists $z$ with $x<z<y$. By Cantor's theorem, every countable dense linear order, which neither has a smallest nor largest element is isomorphic to $\eta$. Hence, if we take symbols 0 and 1 with $0<1$, then $\left(\{0,1\}^{*} 1 ; \leq \operatorname{lex}\right) \cong \eta$. The linear order $\Lambda$ is scattered if there does not exist an injective order morphism $\varphi: \eta \rightarrow \Lambda$. Clearly, $\omega, \bar{\omega}$, as well as every finite linear order are scattered. A linear order is regular if it is isomorphic to a linear order $\left(L ; \leq_{\text {lex }}\right)$ for a regular language $L$. Hence, for instance, $\eta$, $\omega, \bar{\omega}$, and every finite linear order are regular linear orders.

For two linear orders $\Lambda_{1}=\left(L_{1} ; \leq_{1}\right)$ and $\Lambda_{1}=\left(L_{2} ; \leq_{2}\right)$ with $L_{1} \cap L_{2}=\emptyset$ we define the sum $\Lambda_{1}+\Lambda_{2}=\left(L_{1} \cup L_{2} ; \leq\right)$, where $x \leq y$ if and only if either $x, y \in L_{1}$ and $x \leq_{1} y$, or $x, y \in L_{2}$ and $x \leq_{2} y$, or $x \in L_{1}$ and $y \in L_{2}$. We define the product $\Lambda_{1} \cdot \Lambda_{2}=\left(L_{1} \times L_{2} ; \leq\right)$ where $\left(x_{1}, x_{2}\right) \leq\left(y_{1}, y_{2}\right)$ if and only if either $x_{2}<_{2} y_{2}$ or $\left(x_{2}=y_{2}\right.$ and $\left.x_{1} \leq_{1} y_{1}\right)$.

An interval of $\Lambda$ is a subset $I \subseteq L$ such that $x<z<y$ and $x, y \in I$ implies $z \in I$. An interval is right-closed (resp. left-closed) if it has a greatest (resp. smallest) element and it is closed if it is both right-closed and left-closed. An interval I is dense (resp., scattered) if the linear order $\leq$ restricted to $I$ is dense (resp., scattered). A predecessor (resp., successor) of $x \in L$ is a largest (resp., smallest) element of $\{y \in L \mid y<x\}$ (resp., $\{y \in L \mid x<y\}$ ). Of course, a predecessor (resp., successor) of $x$ need not exist, but if it exists then it is unique.

\subsection{Generalized words}

Generalized words are countable colored linear orders. Let $\Sigma$ be a (possibly infinite) alphabet. A generalized word (or simply word) $u$ over $\Sigma$ is a triple $(L ; \leq, \tau)$ such that $L$ is a finite or countably infinite set, $\leq$ is a linear order on $L$ and $\tau: L \rightarrow \Sigma$ is a coloring of $L$. The alphabet alph $(u)$ equals the image of $\tau$. If $L$ is finite, we obtain a finite word in the usual sense. As for trees, we write $u \cong v$ for generalized words $u$ and $v$ in case $u$ and $v$ are isomorphic.

Let $u=(L ; \leq, \tau)$ be a generalized word over $\Sigma$ with $\Gamma=\operatorname{alph}(u)$. Let $v_{a}=$ $\left(L_{a} ; \leq_{a}, \tau_{a}\right)$ be a generalized word for each $a \in \Gamma$. We define the generalized word $u\left[\left(a / v_{a}\right)_{a \in \Gamma}\right]=\left(L^{\prime} ; \leq, \tau^{\prime}\right)$ as follows:

- $L^{\prime}=\left\{(x, y) \mid y \in L, x \in L_{\tau(y)}\right\}$,

- $(x, y) \leq\left(x^{\prime}, y^{\prime}\right)$ if and only if either $y<y^{\prime}$ or $\left(y=y^{\prime}\right.$ and $\left.x \leq_{\tau(y)} x^{\prime}\right)$, and

- $\tau^{\prime}(x, y)=\tau_{\tau(y)}(x)$.

Thus, $u\left[\left(a / v_{a}\right)_{a \in \Gamma}\right]$ is obtained from $u$ by replacing every $a$-labelled point by $v_{a}$ (for all $a \in \Sigma)$. Now we can define the regular operations on words. In order to do so we need the following words. The words $a b$ and $a^{\omega}$ for $a, b \in \Sigma$ are as usual. The generalized word $a^{\bar{\omega}}$ has $\bar{\omega}$ as underlying order and every element is colored with $a$. Finally, we let $\left[a_{1}, \ldots, a_{n}\right]^{\eta}$ be the generalized word with underlying order $\eta$ where the coloring 
is such that any point is labeled by some $a_{i}(1 \leq i \leq n)$ and, moreover, for any two points $x<y$ and any $1 \leq i \leq n$ we find a point $z$ with $x<z<y$ colored by $a_{i}$. It can be shown that this describes a unique word up to isomorphism [26].

Definition 2.3 (Regular Operations). Let $u, v, u_{1}, \ldots, u_{n}$ be words over $\Sigma$. We let:

$$
\begin{aligned}
u v & =(a b)[a / u, b / v] & & u^{\omega}=a^{\omega}[a / u] \\
{\left[u_{1}, \ldots, u_{n}\right]^{\eta} } & =\left[a_{1}, \ldots, a_{n}\right]^{\eta}\left[a_{1} / u_{1}, \ldots, a_{n} / u_{n}\right] & & u^{\bar{\omega}}=a^{\bar{\omega}}[a / u] .
\end{aligned}
$$

Thus, the underlying linear order of $u v$ is the sum of the underlying linear orders of $u$ and $v$. Intuitively, we have $u^{\omega}=u u u \cdots$ and $u^{\omega}=\cdots u u u$. Note that since $\left[u_{1}, \ldots, u_{n}\right]^{\eta}$ is invariant under permutations of the $u_{i}$ we also sometimes use the notation $X^{\eta}$ for a finite set $X$. The least set of words which is closed under the regular operations and contains the singleton words $a$ for $a \in \Sigma$ is called the set of regular words over $\Sigma$, denoted $\operatorname{Reg}(\Sigma)$. Note that this implies that every regular word is non-empty, i.e., its domain is a non-empty set. Moreover, although we allow $\Sigma$ to be infinite (this will be useful later), the alphabet alph $(u)$ of a regular word $u$ must be finite. Clearly, every regular word can be described by a regular expression over the above operations, but this regular expression is in general not unique.

Example 2.4. Here are some typical identities between regular words, where $X$ is a finite set of regular words, $n \geq 0, m \geq 1, u, u_{1}, \ldots, u_{n} \in X$, every $v_{i}(1 \leq i \leq m)$ has one of the forms $X^{\eta}, y X^{\eta}, X^{\eta} z, y X^{\eta} z$ with $y, z \in X$, and $v, w$ are regular words:

$$
\begin{gathered}
X^{\eta} X^{\eta} \cong X^{\eta} u X^{\eta} \cong\left(X^{\eta}\right)^{\omega} \cong\left(X^{\eta} u\right)^{\omega} \cong\left(X^{\eta}\right)^{\bar{\omega}} \cong\left(u X^{\eta}\right)^{\bar{\omega}} \cong X^{\eta}, \\
{\left[u_{1}, \ldots, u_{n}, v_{1}, \ldots, v_{m}\right]^{\eta} \cong X^{\eta}} \\
(v w)^{\omega}=v(w v)^{\omega}, \quad(v w)^{\bar{\omega}}=(w v)^{\bar{\omega}} w .
\end{gathered}
$$

See [2] for a complete axiomatization of the equational theory of regular words.

By a result of Heilbrunner [13], regular words can be characterized by partitioned DFAs as follows: Let $\mathcal{A}=\left(Q, \Gamma, \delta, q_{0},\left(F_{a}\right)_{a \in \Sigma}\right)$ be a partitioned DFA, and let $\mathcal{B}=$ $\left(Q, \Gamma, \delta, q_{0}, \bigcup_{a \in \Sigma} F_{a}\right)$. Let us fix a linear order on the alphabet $\Gamma$, so that the lexicographic order $\leq_{\text {lex }}$ is defined on $\Gamma^{*}$. Then we denote with $w(\mathcal{A})$ the generalized word

$$
w(\mathcal{A})=\left(L(\mathcal{B}) ; \leq_{\text {lex }}, \tau\right),
$$

where $\tau(u)=a(a \in \Sigma, u \in L(\mathcal{B}))$ if and only if $u \in L\left(Q, \Gamma, \delta, q_{0}, F_{a}\right)$. It is easy to construct from a given regular expression (describing the regular word $u$ ) a partitioned DFA $\mathcal{A}$ with $u \cong w(\mathcal{A})$, see e.g. [29, proof of Proposition 2] for a simple construction. The other direction is more difficult. Heilbrunner has shown in [13] how to compute from a given partitioned DFA $\mathcal{A}$ (such that $w(\mathcal{A})$ is non-empty) a regular expression for the word $w(\mathcal{A})$, which is therefore regular. ${ }^{3}$ Unfortunately, the size of the regular expression produced by Heilbrunner's algorithm is exponential in the size of $\mathcal{A}$. In

\footnotetext{
${ }^{3}$ In fact, Heilbrunner speaks about systems of equations and their least solutions instead of partitioned DFAs. But these two formalisms can be easily (and efficiently) transformed into each other.
} 
Section 4.4, we will see that a succinct representation of a regular expression for $w(\mathcal{A})$ can be produced in polynomial time.

One can show that the isomorphism problem for regular words (given by partitioned DFAs) can be reduced (in logspace) to the isomorphism problem for regular linear orders (given by DFAs). In other words, node labels can be eliminated as for regular trees (as remarked at the end of Section 2.3). So, the reader might ask, why we consider the isomorphism problem for regular words and do not restrict to regular linear orders. The point is that even if we start with regular linear orders, in the course of our polynomial isomorphism check regular words will naturally arise.

\section{Isomorphism problem for regular trees}

In this section, we investigate the isomorphism problem for (unordered) regular trees. We consider two input representations for regular trees: DFAs and NFAs. It turns out that while the isomorphism problem for DFA-represented regular trees is P-complete, the same problem becomes EXPTIME-complete for NFA-represented regular trees. Moreover, we show that for finite trees that are succinctly represented by acyclic NFAs, isomorphism is PSPACE-complete.

\subsection{Upper bounds}

Theorem 3.1. The following problem can be solved in polynomial time:

INPUT: Two DFAs $\mathcal{A}_{1}$ and $\mathcal{A}_{2}$ such that $\varepsilon \in L\left(\mathcal{A}_{1}\right) \cap L\left(\mathcal{A}_{2}\right)$.

QUESTION: $\left(L\left(\mathcal{A}_{1}\right) ; \leq_{\text {pref }}\right) \cong\left(L\left(\mathcal{A}_{2}\right) ; \leq_{\text {pref }}\right)$ ?

Proof. By taking the disjoint union of $\mathcal{A}_{1}$ and $\mathcal{A}_{2}$, it suffices to solve the following problem in polynomial time:

INPUT: A DFA $\mathcal{A}$ without initial state and two final states $p, q$ of $\mathcal{A}$. QUESTION: $\left(L(\mathcal{A}, p) ; \leq_{\text {pref }}\right) \cong\left(L(\mathcal{A}, q) ; \leq_{\text {pref }}\right)$ ?

Note that $\varepsilon \in L(\mathcal{A}, p) \cap L(\mathcal{A}, q)$ since $p$ and $q$ are final. Let $\mathcal{A}=(Q, \Sigma, \delta, F)$. In fact, we will compute in polynomial time the equivalence relation

$$
\text { iso }=\left\{(p, q) \in F \times F \mid\left(L(\mathcal{A}, p) ; \leq_{\text {pref }}\right) \cong\left(L(\mathcal{A}, q) ; \leq_{\text {pref }}\right)\right\} .
$$

This will be done similarly to the classical partition refinement algorithm for checking bisimilarity of finite state systems [15].

For $p \in F$ and $C \subseteq F$ let $L(\mathcal{A}, p, C)$ be the set of all words accepted by the DFA $(Q, \Sigma, \delta, p, C)$. Hence, the sets $L(\mathcal{A}, p,\{q\})(q \in F)$ partition $L(\mathcal{A}, p)$. Let us say that a node $u \in L(\mathcal{A}, p)$ is of type $q$ if $u \in L(\mathcal{A}, p,\{q\})$. For $p \in F$ and $C \subseteq F$ let us define the subset $K(\mathcal{A}, p, C) \subseteq L(\mathcal{A}, p, C)$ as the set of all words over $\Sigma$ labeling a path from $p$ to a state from $C$ without intermediate final states; this is clearly a regular language and a DFA for $K(\mathcal{A}, p, C)$ can be easily computed in polynomial time from $\mathcal{A}, p$, and $C$ : We take the DFA $\mathcal{A}$ and remove every transition leaving a final state from $F$. Moreover, we introduce a copy $p^{\prime}$ of $p$, which will be the new initial state and there 
is an $a$-labeled transition from $p^{\prime}$ to $q$ if and only if there is an $a$-labeled transition from $p$ to $q$ in $\mathcal{A}$. Finally, $C$ is the set of final states.

Note that if $u \in L(\mathcal{A}, p)$ is of type $q$, then the nodes $u v$ with $v \in K(\mathcal{A}, q, F)$ are exactly the children of $u$ in the tree $\left(L(\mathcal{A}, p) ; \leq_{\text {pref }}\right)$. Let $n(p, q) \in \mathbb{N} \cup\{\infty\}$ be the cardinality of the language $K(p,\{q\})$. By Lemma 2.2, each of these numbers $n(p, q)$ can be computed in polynomial time. For $C \subseteq F$ let $n(p, C)=\sum_{q \in F} n(p, q)$. Thus $n(p, C)$ is the cardinality of the language $K(p, C)$.

Let us now compute the equivalence relation iso. As already remarked, this will be done by a partition refinement algorithm. Assume that $R$ is an equivalence relation on $F$. We define the new equivalence relation $\widetilde{R}$ on $F$ as follows:

$$
\widetilde{R}=\{(p, q) \in R \mid n(p, C)=n(q, C) \text { for every equivalence class } C \text { of } R\} .
$$

Thus, $\widetilde{R}$ is a refinement of $R$ which can be computed in polynomial time from $R$. Let us define a sequence of equivalence relations $R_{0}, R_{1}, \ldots$ on $F$ as follows: $R_{0}=F \times F$, $R_{i+1}=\widetilde{R}_{i}$. Then, there exists $k<|F|$ such that $R_{k}=R_{k+1}$. We claim that $R_{k}=$ iso. A simple argument shows that for every equivalence relation $R$ on $F$ with iso $\subseteq R$, one has iso $\subseteq \widetilde{R}$ as well. Hence, by induction over $i \geq 0$, one gets iso $\subseteq R_{i}$ for all $i \geq 0$.

For the other direction, we show that if $R$ is an equivalence relation on $F$ such that $R=\widetilde{R}$ (this holds for $R_{k}$ ), then $R \subseteq$ iso. So, assume that $\left(p_{1}, p_{2}\right) \in R=\widetilde{R}$. We will define an isomorphism $f:\left(L\left(\mathcal{A}, p_{1}\right) ; \leq_{\text {pref }}\right) \rightarrow\left(L\left(\mathcal{A}, p_{2}\right) ; \leq_{\text {pref }}\right)$ as the limit of isomorphisms $f_{n}, n \geq 1$. Here, $f_{n}$ is an isomorphism between the trees that result from $\left(L\left(\mathcal{A}, p_{1}\right) ; \leq_{\text {pref }}\right)$ and $\left(L\left(\mathcal{A}, p_{2}\right) ; \leq_{\text {pref }}\right)$ by cutting off all nodes below level $n$ (the roots are one level 1). Let us call these trees $\left.\left(L\left(\mathcal{A}, p_{i}\right) ; \leq_{\text {pref }}\right)\right|_{n}(i \in\{1,2\})$. Moreover, $f_{n}$ has the additional property that if $f_{n}$ maps a node $u_{1}$ of type $q_{1}$ to a node $u_{2}$ of type $q_{2}$, then we will have $\left(q_{1}, q_{2}\right) \in R$. Assume that $f_{n}$ is already constructed and let $u_{1}$ of type $q_{1}$ be a leaf of $\left.\left(L\left(\mathcal{A}, p_{1}\right) ; \leq_{\text {pref }}\right)\right|_{n}$. Let $u_{2}=f\left(u_{1}\right)$ be of type $q_{2}$; it is a leaf of $\left.\left(L\left(\mathcal{A}, p_{2}\right) ; \leq_{\text {pref }}\right)\right|_{n}$. Then we have $\left(q_{1}, q_{2}\right) \in R=\widetilde{R}$ and hence for every equivalence class $C$ of $R$ we have $n\left(q_{1}, C\right)=n\left(q_{2}, C\right)$. We can therefore find a bijection $g$ between the languages $K\left(q_{1}, F\right)$ and $K\left(q_{2}, F\right)$ such that $(u, g(u)) \in R$ for all $u \in K\left(q_{1}, F\right)$. Note that the nodes $u_{i} v$ with $v \in K\left(q_{i}, F\right)$ are the children of $u_{i}$ in the tree $\left(L\left(\mathcal{A}, p_{1}\right) ; \leq_{\text {pref }}\right)$. We now extend the isomorphism $f_{n}$ by $g$ and do this for all leaves $u_{1}$ of $\left.\left(L\left(\mathcal{A}, p_{1}\right) ; \leq_{\text {pref }}\right)\right|_{n}$. This gives us the isomorphism $f_{n+1}$.

Corollary 3.2. The following problem belongs to EXPTIME:

INPUT: Two NFAs $\mathcal{A}_{1}$ and $\mathcal{A}_{2}$ such that $\varepsilon \in L\left(\mathcal{A}_{1}\right) \cap L\left(\mathcal{A}_{2}\right)$.

QUESTION: $\left(L\left(\mathcal{A}_{1}\right) ; \leq_{\text {pref }}\right) \cong\left(L\left(\mathcal{A}_{2}\right) ; \leq_{\text {pref }}\right)$ ?

Proof. In exponential time, we can transform $\mathcal{A}_{1}$ and $\mathcal{A}_{2}$ into DFAs using the powerset construction. Then we can apply Theorem 3.1.

Theorem 3.3. The following problem belongs to PSPACE:

INPUT: Two acyclic NFAs $\mathcal{A}_{1}$ and $\mathcal{A}_{2}$ such that $\varepsilon \in L\left(\mathcal{A}_{1}\right) \cap L\left(\mathcal{A}_{2}\right)$.

QUESTION: $\left(L\left(\mathcal{A}_{1}\right) ; \leq_{\text {pref }}\right) \cong\left(L\left(\mathcal{A}_{2}\right) ; \leq_{\text {pref }}\right)$ ? 
Proof. By [21], isomorphism for finite trees, given explicitly by adjacency lists, can be decided in deterministic logspace. Hence, by Lemma 2.1 it suffices to show that for a given acyclic NFA, the adjacency list representation for the tree $(L(\mathcal{A}) ; \leq$ pref $)$ can be computed by a PSPACE-transducer. This is straightforward. Assume that $\Sigma$ is the alphabet of $\mathcal{A}$ and that $n$ is the number of states of $\mathcal{A}$. Let us fix an arbitrary order on $\Sigma$ and let $z$ be the largest symbol in $\Sigma$.

The language $L(\mathcal{A})$ only contains words of length at most $n-1$. In an outer loop we generate the language $L(\mathcal{A})$. For this, we enumerate all words (e.g. in lexicographic order) of length at most $n-1$ and test whether the current word is accepted by $\mathcal{A}$. For each enumerated word $u \in L(\mathcal{A})$, we have to output a list of all children of $u$ in the tree $\left(L(\mathcal{A}) ; \leq_{\text {pref }}\right)$. In an inner loop, we enumerate (again in lexicographic order) all words $u v\left(v \in \Sigma^{+}\right)$of length at most $n-1$ and check whether $u v \in L(\mathcal{A})$. In case, we find such a word $u v \in L(\mathcal{A})$, we output $u v$ and do the following: If $v \in\{z\}^{+}$, then the inner loop terminates. On the other hand, if $v=v^{\prime} a z^{k}$, where $a \neq z$, then we jump in the inner loop to the word $u v^{\prime} b$, where $b$ is the symbol following $a$ in our order.

\subsection{Lower bounds}

The main result of this section states that the isomorphism problem for regular trees that are represented by NFAs is EXPTIME-hard, which matches the upper bound from the previous section. It is straightforward to prove PSPACE-hardness. If $\Sigma$ is the underlying alphabet of a given NFA $\mathcal{A}$, then $(L(\mathcal{A}) ; \leq$ pref $)$ is a full $|\Sigma|$-ary tree if and only if $L(\mathcal{A})=\Sigma^{*}$. But universality for NFAs is PSPACE-complete [28]. The proof for the EXPTIME lower bound is more involved. Here is a rough outline: EXPTIME coincides with alternating polynomial space [5]. Checking whether a given input is accepted by a polynomial space bounded alternating Turing machine $M$ amounts to evaluate a Boolean expression whose gates correspond to configurations of $M$. Using a construction from [14], the evaluation problem for (finite) Boolean expressions can be reduced to the isomorphism problem for (finite) trees. In our case, the Boolean expression will be infinite. Nevertheless, the infinite Boolean expressions we have to deal with can be evaluated because on every infinite path that starts in the root (the output gate) there will be either an and-gate, where one of the inputs is a false-gate, or an or-gate, where one of the inputs is a true-gate. Applying the construction from [14] to an infinite Boolean expression (that arises from our construction) will yield two infinite trees, which are isomorphic if and only if our Boolean expression evaluates to true. Luckily, these two trees turn out to be regular, and they can be represented by small NFAs.

Infinite Boolean formulas. Let us fix the alphabet

$$
\Omega=\left\{a, \ell_{\wedge}, \ell_{\wedge}^{\prime}, r_{\wedge}, \ell_{\vee}, \ell_{\vee}^{\prime}, r_{\vee}\right\} .
$$

In the following, we will only consider prefix-closed trees over the alphabet $\Omega$ (we will not mention this explicitly all the time). Moreover, we will identify the tree $\left(L ; \leq_{\text {pref }}\right)$ with the language $L$. Now, consider such a tree $T \subseteq \Omega^{*}$. Then, $T$ is well-formed, if the following conditions hold: 
(a) If $u=\varepsilon$ or $u \in T$ ends with $\ell_{\vee}, \ell_{\wedge}, r_{\vee}$, or $r_{\wedge}$, then child $(u, T)$ is one of the following sets, where $\circ \in\{\vee, \wedge\}:\left\{u \ell_{0}, u r_{\circ}\right\},\left\{u \ell_{\circ}^{\prime}, u r_{\circ}\right\},\left\{u a, u \ell_{0}^{\prime}, u r_{\circ}\right\}$.

(b) If $u \in T$ ends with $a, \ell_{\vee}^{\prime}$, or $\ell_{\wedge}^{\prime}$, then $u$ is a leaf of $T$.

(c) For every infinite path $P$ in $T$, there exists $u \in P$ with $u a \in T$.

Note that a well-formed tree $T$ is always infinite; it contains an infinite path of the form $r_{1} r_{2} r_{3} \cdots$, where $r_{i} \in\left\{r_{\wedge}, r_{\vee}\right\}$ for all $i \geq 1$. Let us define the set

$$
\operatorname{cut}(T)=\left\{u \in T \mid u a \in T, \forall v<_{\text {pref }} u: v a \notin T\right\} .
$$

Hence, on every infinite path in $T$ there is a unique node from cut $(T)$.

With a well-formed tree $T$ we associate an infinite Boolean expression bool $(T)$ as follows: The gates of bool $(T)$ are the nodes of $T$ that do not end with $a$.

- The set of input gates for $u \in T$ is $\operatorname{child}(u, T) \backslash\{u a\}$.

- If $u r_{\vee} \in T$ (resp. $u r_{\wedge} \in T$ ), then $u$ is an or-gate (resp. and-gate).

- If $u \ell_{\wedge}^{\prime} \in T$ and $u a \notin T$, then $u \ell_{\wedge}^{\prime}$ is a true-gate.

- If $u \ell_{\wedge}^{\prime} \in T$ and $u a \in T$, then $u \ell_{\wedge}^{\prime}$ is a false-gate.

- If $u \ell_{\vee}^{\prime} \in T$ and $u a \notin T$, then $u \ell_{\vee}^{\prime}$ is a false-gate.

- If $u \ell_{\vee}^{\prime} \in T$ and $u a \in T$, then $u \ell_{\vee}^{\prime}$ is a true-gate.

Although bool $(T)$ is an infinite Boolean formula, the fact that $T$ is well-formed ensures that the root of bool $(T)$ can be evaluated: We simply remove from $T$ all nodes that have a proper prefix from cut $(T)$. The resulting tree has no infinite path and since it is finitely branching it is finite by König's lemma. If $u \in \operatorname{cut}(T)$ is such that $u \ell_{\wedge}^{\prime} \in T$ (resp., $u \ell_{\vee}^{\prime} \in T$ ), then $u$ can be transformed into a false-gate (resp., true-gate). Then, one has to evaluate the resulting finite Boolean expression.

We next transform a tree $T \subseteq \Omega^{*}$ into trees $[T]_{1},[T]_{2} \subseteq\{\ell, r\}^{*}$ using two rational transducers. These two transducers only differ in their initial state. For $i \in\{1,2\}$, let $\mathcal{T}_{i}$ be the transducer from Figure 1, where the initial state is $q_{i}$ and all states are final. Then, for a tree $T \subseteq \Omega^{*}$ and $i \in\{1,2\}$ let $[T]_{i}=\operatorname{pref}\left(\mathcal{T}_{i}(T)\right)$. We will show that for every well-formed tree $T \subseteq \Omega^{*}$ : bool $(T)$ evaluates to true if and only if $[T]_{1} \cong[T]_{2}$. (Lemma 3.9) For this, we first have to show a few lemmas.

Lemma 3.4. Let $T=\left\{\varepsilon, \ell_{\vee}^{\prime}\right\} \cup r_{\vee} U$ or $T=\left\{\varepsilon, \ell_{\wedge}^{\prime}\right\} \cup r_{\wedge} U$ for a tree $U$ (hence, also $T$ is a tree). Then $[T]_{1} \cong[T]_{2}$ if and only if $[U]_{1} \cong[U]_{2}$.

Proof. We only prove the lemma for $T=\left\{\varepsilon, \ell_{\vee}^{\prime}\right\} \cup r_{\vee} U$; the statement for $T=$ $\left\{\varepsilon, \ell_{\wedge}^{\prime}\right\} \cup r_{\wedge} U$ can be shown analogously. Let us compute compute $\mathcal{T}_{1}(T)$ and $\mathcal{T}_{2}(T)$. We have

$$
\mathcal{T}_{1}\left(\ell_{\vee}^{\prime}\right)=\mathcal{T}_{2}\left(\ell_{\vee}^{\prime}\right)=\left\{\ell^{2}, r \ell^{2}\right\} .
$$

Next, we have to compute $\mathcal{T}_{1}\left(r_{\vee} U\right)$. There are two transitions starting in $q_{1}$, where $r_{\vee}$ can be read, namely

$$
q_{1} \stackrel{r_{\vee} \mid \ell r \ell}{\longrightarrow} q_{2} \quad \text { and } \quad q_{1} \stackrel{r_{\vee} \mid r^{2} \ell}{\longrightarrow} q_{1}
$$

Hence, we get

$$
\mathcal{T}_{1}\left(r_{\vee} U\right)=r^{2} \ell \mathcal{T}_{1}(U) \cup \ell r \ell \mathcal{T}_{2}(U)
$$




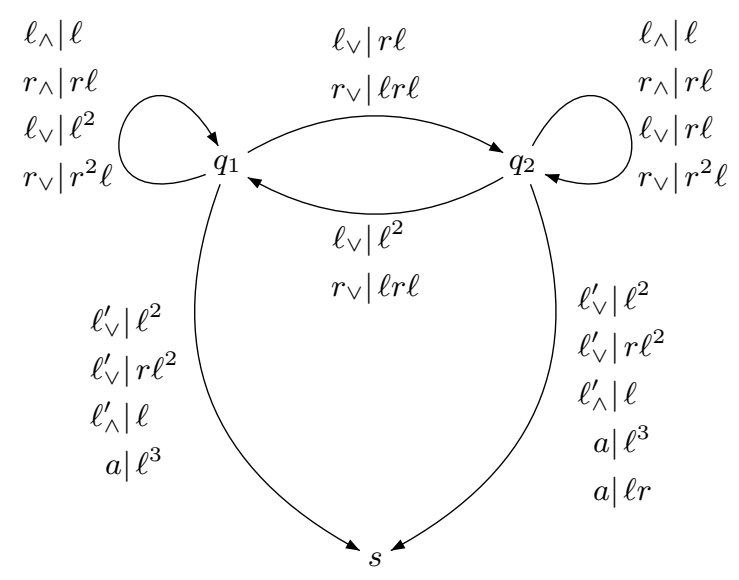

Fig. 1. The transducer
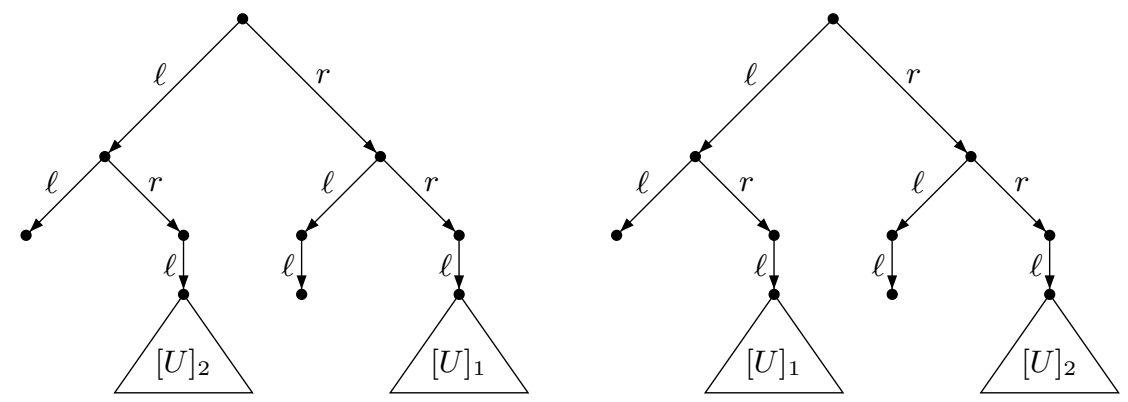

Fig. 2. $[T]_{1}$ (left) and $[T]_{2}$ (right) from Lemma 3.4

Similarly, we get

$$
\mathcal{T}_{2}\left(r_{\vee} U\right)=r^{2} \ell \mathcal{T}_{2}(U) \cup \ell r \ell \mathcal{T}_{1}(U)
$$

From (3), (4), and (5) it follows that the trees $[T]_{i}=\operatorname{pref}\left(\mathcal{T}_{i}\left(\left\{\varepsilon, \ell_{\vee}^{\prime}\right\} \cup r_{\vee} U\right)\right)(i \in$ $\{1,2\})$ are the ones shown in Figure 2 . The equivalence of $[T]_{1} \cong[T]_{2}$ and $[U]_{1} \cong[U]_{2}$ is obvious from these diagrams.

The following three lemmas can be shown with the same kinds of arguments as for Lemma 3.4. We therefore only sketch the proofs.

Lemma 3.5. Let $T=\left\{\varepsilon, \ell_{\vee}^{\prime}, a\right\} \cup r_{\vee} U$ for a tree $U$ (hence, also $T$ is a tree). Then $[T]_{1} \cong[T]_{2}$.

Proof. We have $\mathcal{T}_{1}(a)=\left\{\ell^{3}\right\}$ and $\mathcal{T}_{2}(a)=\left\{\ell^{3}, \ell r\right\}$. It follows, that the trees $[T]_{1}$ and $[T]_{2}$ are as shown in Figure 3. Clearly, we have $[T]_{1} \cong[T]_{2}$. 

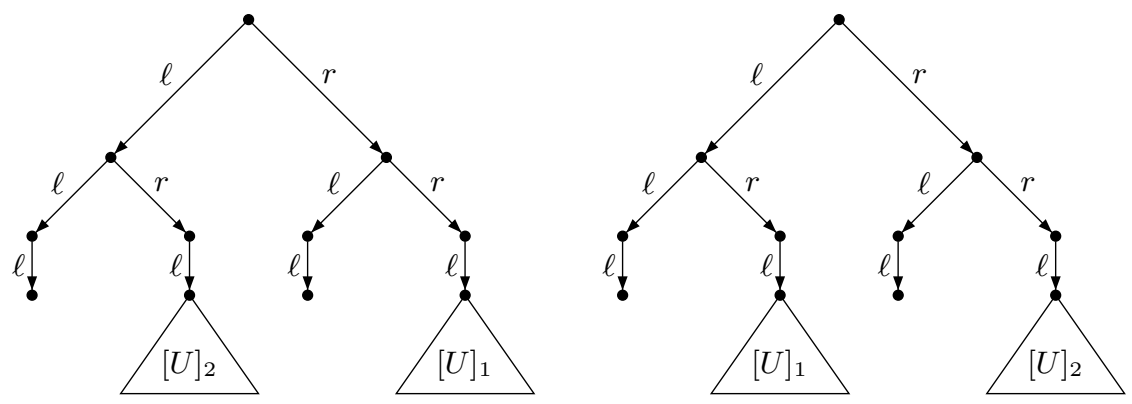

Fig. 3. $[T]_{1}$ (left) and $[T]_{2}$ (right) from Lemma 3.5
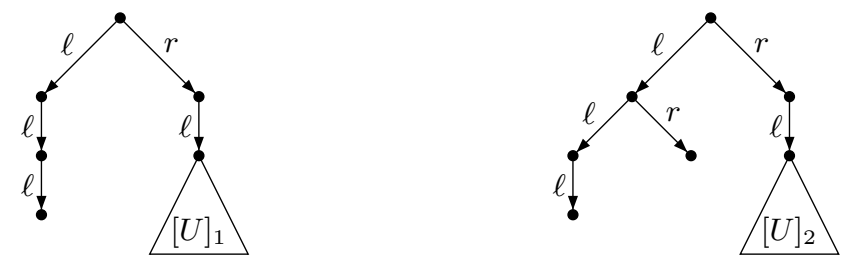

Fig. 4. $[T]_{1}$ (left) and $[T]_{2}$ (right) from Lemma 3.6

Lemma 3.6. Let $T=\left\{\varepsilon, \ell_{\wedge}^{\prime}, a\right\} \cup r_{\wedge} U$ for a tree $U$ (hence, also $T$ is a tree). Then $[T]_{1} \neq[T]_{2}$.

Proof. The trees $[T]_{1}$ and $[T]_{2}$ are shown in Figure 4. Clearly, we have $[T]_{1} \not \equiv[T]_{2}$.

Lemma 3.7. Let $T=\{\varepsilon\} \cup \ell_{\vee} U \cup r_{\vee} V$ for well-formed trees $U, V$ (hence, also $T$ is well-formed $)$. Then $[T]_{1} \cong[T]_{2}$ if and only if $\left([U]_{1} \cong[U]_{2}\right.$ or $\left.[V]_{1} \cong[V]_{2}\right)$.

Proof. The trees $[T]_{1}$ and $[T]_{2}$ are shown in Figure 5. Since $U$ and $V$ are well-formed, in each of the trees $[U]_{1},[U]_{2},[V]_{1}$, and $[V]_{2}$, the root has two children. It follows easily that $[T]_{1} \cong[T]_{2}$ if and only if $\left([U]_{1} \cong[U]_{2}\right.$ or $\left.[V]_{1} \cong[V]_{2}\right)$.

Lemma 3.8. Let $T=\{\varepsilon\} \cup \ell_{\wedge} U \cup r_{\wedge} V$ for well-formed trees $U, V$ (hence, also $T$ is well-formed). Then $[T]_{1} \cong[T]_{2}$ if and only if $\left([U]_{1} \cong[U]_{2}\right.$ and $\left.[V]_{1} \cong[V]_{2}\right)$.

Proof. The trees $[T]_{1}$ and $[T]_{2}$ are as shown in Figure 6. Since $U$ and $V$ are wellformed, in each of the trees $[U]_{1},[U]_{2},[V]_{1}$, and $[V]_{2}$, the root has two children. It follows easily that $[T]_{1} \cong[T]_{2}$ if and only if $\left([U]_{1} \cong[U]_{2}\right.$ and $\left.[V]_{1} \cong[V]_{2}\right)$.

Lemma 3.9. For every well-formed tree $T \subseteq \Omega^{*}$, we have: bool $(T)$ evaluates to true if and only if $[T]_{1} \cong[T]_{2}$. 

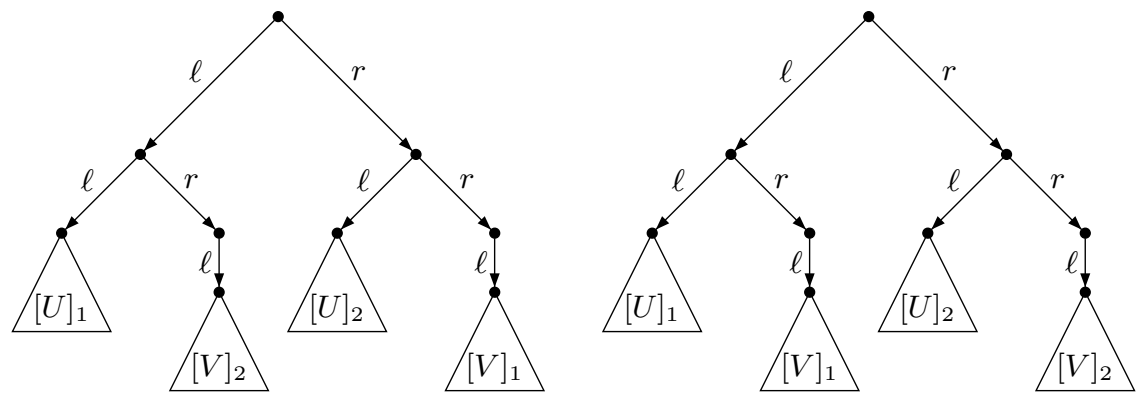

Fig. 5. $[T]_{1}$ (left) and $[T]_{2}$ (right) from Lemma 3.7
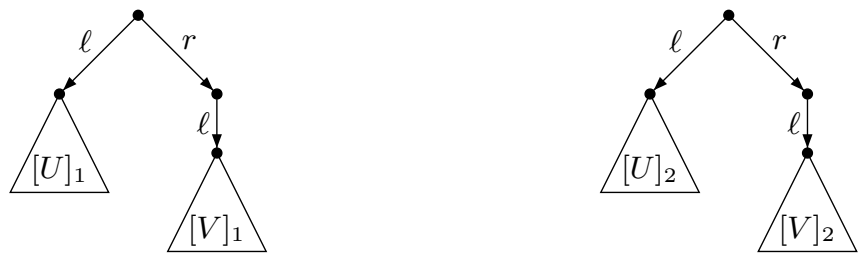

Fig. 6. $[T]_{1}$ (left) and $[T]_{2}$ (right) from Lemma 3.8

Proof. Recall the definition of the set cut $(T)$ from (2). From the definition it follows that $\operatorname{pref}(\operatorname{cut}(T))$ is a finitely branching tree without infinite paths. Hence, by König's lemma it is finite. Moreover, for every $u \in \operatorname{pref}(\operatorname{cut}(T))$, the subtree $T \uparrow_{u}$ is wellformed as well (since $\left.\operatorname{pref}(\operatorname{cut}(T)) \subseteq\{\varepsilon\} \cup \Omega^{*}\left\{\ell_{\vee}, \ell_{\wedge}, r_{\vee}, r_{\wedge}\right\}\right)$. Inductively over the height of $u \in \operatorname{pref}(\operatorname{cut}(T))$ in the finite tree $\operatorname{pref}(\operatorname{cut}(T))$, we will prove for every $u \in \operatorname{pref}(\operatorname{cut}(T)):\left[T \uparrow_{u}\right]_{1} \cong\left[T \uparrow_{u}\right]_{2}$ if and only if bool $\left(T \uparrow_{u}\right)$ evaluates to true.

For the induction base, let $u \in \operatorname{cut}(T)$ be a leaf of $\operatorname{pref}(\operatorname{cut}(T))$. Hence, we have $u a \in T$. If $u \ell_{\wedge}^{\prime} \in T$, then in bool $\left(T \uparrow_{u}\right)$, the root is an and-gate for which one of the inputs (namely $u \ell_{\wedge}^{\prime}$ ) is a false-gate. Hence, bool $\left(\left.T\right|_{u}\right)$ evaluates to false. Moreover, Lemma 3.6 implies that $\left[T \uparrow_{u}\right]_{1} \neq\left[T \uparrow_{u}\right]_{2}$. On the other hand, if $u \ell_{\vee}^{\prime} \in T$, then in $\operatorname{bool}\left(T \uparrow_{u}\right)$, the root is an or-gate for which one of the inputs (namely $u \ell_{\vee}^{\prime}$ ) is a truegate. Hence, bool $\left(T \uparrow_{u}\right)$ evaluates to true. Moreover, Lemma 3.5 implies that $\left[T \uparrow_{u}\right]_{1} \cong$ $\left[T \uparrow_{u}\right]_{2}$. This concludes the induction base.

Next, let $u \in \operatorname{pref}(\operatorname{cut}(T))$ be a proper prefix of a node from cut $(T)$. In particular $u \notin \operatorname{cut}(T)$. We can distinguish 4 different cases: 
Case 1. $\operatorname{child}(u, T)=\left\{u \ell_{\wedge}, u r_{\wedge}\right\}$. We must have $\left\{u \ell_{\wedge}, u r_{\wedge}\right\} \subseteq \operatorname{pref}(\operatorname{cut}(T))$. Hence, the induction hypothesis (IH) holds for $u \ell_{\wedge}$ and $u r_{\wedge}$. We get:

$$
\begin{aligned}
& \operatorname{bool}\left(T \uparrow_{u}\right) \text { evaluates to true } \Longleftrightarrow \operatorname{bool}\left(T \uparrow_{u \ell_{\curlywedge}}\right) \text { evaluates to true and } \\
& \operatorname{bool}\left(T \uparrow_{u r_{\wedge}}\right) \text { evaluates to true } \\
& \stackrel{(\mathrm{IH})}{\Longleftrightarrow}\left[T \uparrow_{u \ell_{\wedge}}\right]_{1} \cong\left[T \uparrow_{u \ell_{\wedge}}\right]_{2} \text { and } \\
& {\left[T \uparrow_{u r_{\wedge}}\right]_{1} \cong\left[T \uparrow_{u r_{\wedge}}\right]_{2}} \\
& \stackrel{\text { Lemma }}{\Longleftrightarrow}{ }^{3.8}\left[T \uparrow_{u}\right]_{1} \cong\left[T \uparrow_{u}\right]_{2}
\end{aligned}
$$

Case 2. $\operatorname{child}(u, T)=\left\{u \ell_{\vee}, u r_{\vee}\right\}$. This case is analogous to Case 1, using Lemma 3.7.

Case 3. $\operatorname{child}(u, T)=\left\{u \ell_{\wedge}^{\prime}, u r_{\wedge}\right\}$. Since $u \notin \operatorname{cut}(T)$, we have $u a \notin T$. We must have $u r_{\wedge} \in \operatorname{pref}(\operatorname{cut}(T))$. Moreover, in bool $\left(T \uparrow_{u}\right)$, the root is an and-gate, where one of the inputs is a true-gate and the other input is the root for the Boolean expression $\operatorname{bool}\left(T \uparrow_{u r_{\wedge}}\right)$. Hence, we get:

$$
\begin{aligned}
& \text { bool }\left(T \uparrow_{u}\right) \text { evaluates to true } \Longleftrightarrow \operatorname{bool}\left(T \uparrow_{u r_{\wedge}}\right) \text { evaluates to true } \\
& \stackrel{(\mathrm{IH})}{\Longleftrightarrow}\left[T \uparrow_{u r_{\wedge}}\right]_{1} \cong\left[T \uparrow_{u r_{\wedge}}\right]_{2} \\
& \stackrel{\text { Lemma }}{\Longleftrightarrow .4}\left[T \uparrow_{u}\right]_{1} \cong\left[T \uparrow_{u}\right]_{2}
\end{aligned}
$$

Case 4. $\operatorname{child}(u, T)=\left\{u \ell_{\vee}^{\prime}, u r_{\vee}\right\}$. This case is analogous to Case 3 .

Our last auxiliary lemma states that an NFA for the tree $[L]_{i}$ can be easily computed from an NFA for $L$.

Lemma 3.10. There is a logspace machine that computes from a given prefix-closed $N F A \mathcal{A}$ with terminal alphabet $\Omega$ a prefix-closed NFA $\mathcal{B}$ such that $L(\mathcal{B})=[L(\mathcal{A})]_{i}$ for $i \in\{1,2\}$.

Proof. Let $\mathcal{A}=\left(Q, \Omega, \delta, p_{0}, Q\right)$. Recall that all states of $\mathcal{T}_{i}$ and $\mathcal{A}$ are final. The prefixclosed NFA $\mathcal{B}$ is obtained from the direct product of $\mathcal{A}$ and $\mathcal{T}_{i}$ by adding further states so that every transition is labeled with a single symbol. Thus, the set of states of $\mathcal{B}$ contains $Q \times\left\{q_{1}, q_{2}, s\right\}$ and the initial state of $\mathcal{B}$ is $\left(p_{0}, q_{i}\right)$. If $q \stackrel{b}{\rightarrow} q^{\prime}$ in $\mathcal{A}$ and $t \stackrel{b \mid w}{\longrightarrow} t^{\prime}$ in $\mathcal{T}_{i}$ for $w \in\{\ell, r\}^{+}$, then we add $|w|-1$ many new states to $\mathcal{B}$, which built up a $w$-labeled path from from $(q, t)$ to $\left(q^{\prime}, t^{\prime}\right)$.

EXPTIME-hardness. We are now in the position to prove the main result of this section.

Theorem 3.11. The following problem is EXPTIME-hard (and hence EXPTIME-complete):

INPUT: Two prefix-closed NFAs $\mathcal{A}_{1}$ and $\mathcal{A}_{2}$.

QUESTION: $\left(L\left(\mathcal{A}_{1}\right) ; \leq_{\text {pref }}\right) \cong\left(L\left(\mathcal{A}_{2}\right) ; \leq_{\text {pref }}\right)$ ? 
Proof. The upper bound is stated in Corollary 3.2. For the lower bound we use the fact that EXPTIME equals the class of all sets that can be accepted in polynomial space on an alternating Turing machine [5]. Hence, let $M$ be a polynomial space bounded alternating Turing machine such that the accepted language $L(M) \subseteq\{0,1\}^{*}$ is EXPTIMEcomplete. We can assume that $M$ has no infinite computation paths. By padding inputs, we can moreover assume that $M$ works in space $n$ for an input of length $n$. Let $Q=Q_{\exists} \cup Q_{\forall}$ be the set of states of $M$ and let $\Gamma \supseteq\{0,1\}$ be the tape alphabet. W.l.o.g. we can assume that in every computation step, $M$ moves from an existential state to a universal state or vice versa, and that the initial state $q_{0}$ is universal.

Let us now fix an input $w \in\{0,1\}^{*}$ of length $n$. We will construct two prefix-closed NFAs $\mathcal{A}_{1}$ and $\mathcal{A}_{2}$ such that $w \in L(M)$ if and only if $\left(L\left(\mathcal{A}_{1}\right) ; \leq\right.$ pref $) \cong\left(L\left(\mathcal{A}_{2}\right) ; \leq_{\text {pref }}\right)$. Let $\Theta=\Gamma \cup Q$. As usual, a configuration of $M$ can be represented by a string from the language $\Theta^{n+1}$ (more precisely, from $\bigcup_{j=0}^{n-1} \Gamma^{j} Q \Gamma^{n-j}$ ). A word $u \in \Theta^{*}$ is a valid computation of $M$ on input $w$ if $u$ is of the form $c_{1} \cdots c_{m}$ for some $m \geq 0$ such that the following holds:

$$
\begin{aligned}
& \text { - } c_{i} \in \bigcup_{j=0}^{n-1} \Gamma^{j} Q \Gamma^{n-j} \text { for all } 1 \leq i \leq m \\
& -c_{i} \vdash_{M} c_{i+1} \text { (i.e., } c_{i+1} \text { is a successor configuration of } c_{i} \text { ) for all } 1 \leq i \leq m-1 \\
& -q_{0} w \vdash_{M} c_{1}
\end{aligned}
$$

Note that $\varepsilon$ is a valid computation in this sense. It is well known that from $w$ one can construct in logspace a coaccessible NFA $\mathcal{A}_{w}$ such that $\mathcal{A}_{w}$ accepts all words over $\Theta$ that are not a valid computation of $M$ on $w$ [28].

Next, we will define a regular well-formed tree $T_{w} \subseteq \Omega^{*}$ (depending only on $w$ ) such that bool $\left(T_{w}\right)$ evaluates to true if and only if $w \in L(M)$. In the following, we identify the symbols in $\Theta$ with the integers $0, \ldots,|\Theta|-1$ in an arbitrary way. We can assume that $|\Theta| \geq 2$. We define two morphisms

$$
\begin{aligned}
& \varphi_{\wedge}: \Theta^{*} \rightarrow\left\{\ell_{\wedge}, r_{\wedge}\right\}^{*} \\
& \varphi_{\vee}: \Theta^{*} \rightarrow\left\{\ell_{\vee}, r_{\vee}\right\}^{*}
\end{aligned}
$$

as follows $(\circ \in\{\wedge, \vee\})$ :

$$
\varphi_{\circ}(a)= \begin{cases}r_{\circ}^{a} \ell_{\circ} & \text { if } 0 \leq a<|\Theta|-1 \\ r_{\circ}^{a} & \text { if } a=|\Theta|-1\end{cases}
$$

For $i \geq 1$, let $\varphi_{i}$ be the mapping $\varphi_{\wedge}$ (resp. $\varphi_{\vee}$ ) if $i$ is odd (resp., even). Similarly, for $x \in\left\{\ell, \ell^{\prime}, r\right\}$, let $x_{i}$ be $x_{\wedge}$ (resp. $x_{\vee}$ ) if $i$ is odd (resp., even). Then, the tree $T_{w} \subseteq \Omega^{*}$ is $\operatorname{pref}\left(T_{w}^{\prime}\right)$, where

$$
\begin{aligned}
T_{w}^{\prime}= & \left\{\left(\prod_{i=1}^{m} r_{i} \varphi_{i}\left(c_{i}\right)\right) \ell_{m+1}^{\prime} \mid m \geq 0, c_{1}, \ldots, c_{m} \in \Theta^{n+1}\right\} \cup \\
& \left\{\left(\prod_{i=1}^{m} r_{i} \varphi_{i}\left(c_{i}\right)\right) a \mid m \geq 0, c_{1}, \ldots, c_{m} \in \Theta^{n+1}, c_{1} \cdots c_{m} \in L\left(\mathcal{A}_{w}\right)\right\}
\end{aligned}
$$

Clearly, $T_{w}$ is regular, and a prefix-closed NFA for $T_{w}$ can be computed in logspace from $w$ (using the logspace computable coaccessible NFA $\mathcal{A}_{w}$ ). 
Claim 1: $T_{w}$ is well-formed.

Proof of Claim 1: The first three conditions for well-formed trees are easy to check. For the last condition, we have to consider an arbitrary infinite path $P$ of $T_{w}$ and show that there exists $u \in T_{w}$ such that $u a \in T$. But this means that $u$ is of the form

$$
u=\prod_{i=1}^{m} r_{i} \varphi_{i}\left(c_{i}\right)
$$

with $m \geq 0, c_{1}, \ldots, c_{m} \in \Theta^{n+1}$, and $c_{1} \cdots c_{m} \in L\left(\mathcal{A}_{w}\right)$. The latter condition means that $c_{1} \cdots c_{m}$ is not a valid computation of $M$ on input $w$. Claim 1 now follows from the fact that for every infinite sequence $c_{1} c_{2} c_{3} \cdots$ with $c_{i} \in \Theta^{n+1}$ for $i \geq 1$ there exists $m \geq 1$ such that $c_{1} \cdots c_{m}$ is not a valid computation of $M$ on input $w$ (since $M$ does not have infinite computation paths).

Claim 2: $w \in L(M)$ if and only if bool $\left(T_{w}\right)$ evaluates to true.

Proof of Claim 2: Let us consider the finite tree $\operatorname{pref}\left(\operatorname{cut}\left(T_{w}\right)\right)$. For every node

$$
g=r_{\wedge} \varphi_{\wedge}\left(c_{1}\right) r_{\vee} \varphi_{\vee}\left(c_{2}\right) r_{\wedge} \cdots \varphi_{m-1}\left(c_{m-1}\right) r_{m} \varphi_{m}\left(c_{m}\right) \in \operatorname{pref}\left(\operatorname{cut}\left(T_{w}\right)\right)
$$

with $m \geq 0$ and $c_{1}, \ldots, c_{m} \in \Theta^{n+1}$ we will prove (by induction on the height of $g$ ) the following: If $c_{1} \cdots c_{m}$ is a valid computation of $M$ on input $w$, then $c_{m}$ is an accepting configuration if and only if $g$ evaluates to true in bool $\left(T_{w}\right)$. Here, for $m=0$, we define $c_{0}$ as the initial configuration $q_{0} w$.

So, assume that $g \in \operatorname{pref}\left(\operatorname{cut}\left(T_{w}\right)\right)$ is of the above form and that $c_{1} \cdots c_{m}$ is a valid computation of $M$ on input $w$. W.l.o.g. assume that $m$ is odd (the case that $m$ is even can be dealt analogously). Thus,

$$
g=r_{\wedge} \varphi_{\wedge}\left(c_{1}\right) r_{\vee} \varphi_{\vee}\left(c_{2}\right) r_{\wedge} \cdots \varphi_{\vee}\left(c_{m-1}\right) r_{\wedge} \varphi_{\wedge}\left(c_{m}\right) .
$$

Then, in bool $\left(T_{w}\right)$, the input gates for the or-gate $g$ are $g \ell_{\vee}^{\prime}$ and $g r_{\vee}$. Since $c_{1} \cdots c_{m}$ is a valid computation of $M$ on input $w, g a$ does not belong to the tree $T_{w}$. Hence, in bool $\left(T_{w}\right), g \ell_{\vee}^{\prime}$ is a false-gate. Thus, $g$ evaluates to true if and only if $g r_{\vee}$ evaluates to true. From the structure of $T_{w}$ we see that the latter holds if and only if there exists $c_{m+1} \in \Theta^{n+1}$ such that $g r_{\vee} \varphi_{\vee}\left(c_{m+1}\right)$ evaluates to true. First assume that $c_{m+1}$ is such that $c_{1} \cdots c_{m} c_{m+1}$ is not a valid computation. The inputs for the andgate $g r_{\vee} \varphi_{\vee}\left(c_{m+1}\right)$ are $g r_{\vee} \varphi_{\vee}\left(c_{m+1}\right) \ell_{\wedge}^{\prime}$ and $g r_{\vee} \varphi_{\vee}\left(c_{m+1}\right) r_{\wedge}$. Since $c_{1} \cdots c_{m} c_{m+1}$ is not a valid computation, $g r_{\vee} \varphi_{\vee}\left(c_{m+1}\right) a$ belongs to the tree $T_{w}$. Thus, in bool $\left(T_{w}\right)$, $g r_{\vee} \varphi_{\vee}\left(c_{m+1}\right) \ell_{\wedge}^{\prime}$ is a false-gate and $g r_{\vee} \varphi_{\vee}\left(c_{m+1}\right)$ evaluates to false. This holds for all $c_{m+1}$ such that $c_{1} \cdots c_{m} c_{m+1}$ is not a valid computation. Hence, $g r_{\vee}$ evaluates to true if and only if there exists a configuration $c_{m+1} \in \Theta^{n+1}$ such that $c_{1} \cdots c_{m} c_{m+1}$ is a valid computation (which means that $c_{m+1}$ is a successor configuration of $c_{m}$ ) and $\operatorname{gr}_{\vee} \varphi_{\vee}\left(c_{m+1}\right)$ evaluates to true in bool $\left(T_{w}\right)$. Now, if $c_{1} \cdots c_{m} c_{m+1}$ is a valid computation, then by induction, $g r_{\vee} \varphi_{\vee}\left(c_{m+1}\right)$ (which belongs to $\operatorname{pref}\left(\operatorname{cut}\left(T_{w}\right)\right)$ as well) evaluates to true in bool $\left(T_{w}\right)$ if and only if $c_{m+1}$ is an accepting configuration of $M$.

We have shown that $g$ evaluates to true if and only if $c_{m}$ has an accepting successor configuration. Finally, since $m$ is odd, $c_{m}$ is an existential configuration (recall that the 
initial configuration $c_{0}=q_{0} w$ is universal). Thus, indeed, $g$ evaluates to true if and only if $c_{m}$ is accepting. This proves Claim 2.

Let $\mathcal{T}_{1}$ and $\mathcal{T}_{2}$ be the rational transducers from Section 3.2. Using Lemma 3.10 we can compute in logspace from a prefix-closed NFA for $T_{w}$ two prefix-closed NFAs $\mathcal{A}_{1}$ and $\mathcal{A}_{2}$ such that $L\left(\mathcal{A}_{i}\right)=\left[T_{w}\right]_{i}$ for $i \in\{1,2\}$. By Lemma 3.9 and Claim 2, we have

$w \in L(M) \Longleftrightarrow \operatorname{bool}\left(T_{w}\right)$ evaluates to true $\Longleftrightarrow\left(L\left(\mathcal{A}_{1}\right) ; \leq_{\text {pref }}\right) \cong\left(L\left(\mathcal{A}_{2}\right) ; \leq_{\text {pref }}\right)$.

This concludes the proof of the EXPTIME lower bound.

\section{PSPACE-hardness}

Theorem 3.12. The following problem is PSPACE-hard (and therefore PSPACE-complete):

INPUT: Two prefix-closed acyclic NFAs $\mathcal{A}_{1}$ and $\mathcal{A}_{2}$.

QUESTION: $\left(L\left(\mathcal{A}_{1}\right) ; \leq_{\text {pref }}\right) \cong\left(L\left(\mathcal{A}_{2}\right) ; \leq_{\text {pref }}\right)$ ?

Proof. The upper bound is stated in Theorem 3.3. For the lower bound, we use the same idea as in the proof of Theorem 3.11. In fact, we will use most of the notations from that proof; some of them will be slightly modified. This time, we use the fact that PSPACE equals the class of all sets that can be accepted in polynomial time on an alternating Turing machine. Hence, let $M$ be a polynomial time bounded alternating Turing machine such that the accepted language $L(M) \subseteq\{0,1\}^{*}$ is PSPACE-complete. Let $p(n)$ (a polynomial) be the time bound and let $q(n)=p(n)+1$. We can assume that $q(n)$ is odd for all $n \geq 0$. W.l.o.g. we can assume again that $M$ works in space $n$ for an input of length $n$. Let $w \in\{0,1\}^{*}$ be an input for $M$ of length $n$.

Let us add to the alphabet $\Omega$ in (1) an additional symbol $r_{\vee}^{\prime}$. The notions from Section 3.2 have to be extended to this new alphabet $\Omega$. In condition (a) for the definition of a well-formed tree $T$, we also allow the set $\left\{u a, u \ell_{\vee}^{\prime}, u r_{\vee}^{\prime}\right\}$ for $\operatorname{child}(u, T)$. Moreover, every node $u r_{\vee}^{\prime} \in T$ is a leaf of $T$. The new definition for the set cut $(T)$ can be overtaken from (2). Also the Boolean expression bool $(T)$ can be defined as in Section 3.2; the truth value of a leaf ending with $r_{\vee}^{\prime}$ is set arbitrarily (say true). Finally, let us extend the two transducers $\mathcal{T}_{1}$ and $\mathcal{T}_{2}$ such that, from $q_{1}$ and $q_{2}$ they can read the new symbol $r_{\vee}^{\prime}$ and output $\ell$ and then terminate in a sink state $s$.

We now define the well-formed tree $U_{w} \subseteq \Omega^{*}$ as $U_{w}=\operatorname{pref}\left(U_{w}^{\prime}\right)$, where:

$$
\begin{aligned}
U_{w}^{\prime}= & \left\{\left(\prod_{i=1}^{m} r_{i} \varphi_{i}\left(c_{i}\right)\right) \ell_{m+1}^{\prime} \mid 0 \leq m \leq q(n), c_{1}, \ldots, c_{m} \in \Theta^{n+1}\right\} \cup \\
& \left\{\left(\prod_{i=1}^{m} r_{i} \varphi_{i}\left(c_{i}\right)\right) a \mid 0 \leq m \leq q(n), c_{1}, \ldots, c_{m} \in \Theta^{n+1}, c_{1} \cdots c_{m} \in L\left(\mathcal{A}_{w}\right)\right\} \cup \\
& \left\{\left(\prod_{i=1}^{q(n)} r_{i} \varphi_{i}\left(c_{i}\right)\right) r_{\vee}^{\prime} \mid c_{1}, \ldots, c_{q(n)} \in \Theta^{n+1}\right\} .
\end{aligned}
$$

Note that $U_{w}$ is finite. An acyclic prefix-closed NFA for $U_{w}$ can be produced in logspace from $w$. Moreover, since every word from $\Theta^{(n+1) q(n)}$ is not a valid computation (since 
$M$ terminates after $\leq p(n)=q(n)-1$ steps $)$, the Boolean expression bool $\left(U_{w}\right)$ and bool $\left(T_{w}\right)$ (where $T_{w}$ was defined in the proof of Theorem 3.11) evaluate to the same truth value. Hence, using Claim 2 from the proof of Theorem 3.11, it follows that $w \in$ $L(M)$ if and only if bool $\left(U_{w}\right)$ evaluates to true. Using an analogon of Lemma 3.9, this holds if and only if $\left[U_{w}\right]_{1} \cong\left[U_{w}\right]_{2}$. Acyclic NFAs for $\left[U_{w}\right]_{1}$ and $\left[U_{w}\right]_{2}$ can be easily constructed in logspace from $w$ (using an acyclic NFA for $U_{w}$ ). This concludes the proof of the theorem.

\section{P-hardness}

Theorem 3.13. The following problem is $\mathrm{P}$-hard (and hence $\mathrm{P}$-complete):

INPUT: Two prefix-closed acyclic DFAs $\mathcal{A}_{1}$ and $\mathcal{A}_{2}$. QUESTION: $\left(L\left(\mathcal{A}_{1}\right) ; \leq_{\text {pref }}\right) \cong\left(L\left(\mathcal{A}_{2}\right) ; \leq_{\text {pref }}\right)$ ?

Proof. The upper bound is stated in Theorem 3.1. For the lower bound, we reduce the $\mathrm{P}$-complete monotone circuit value problem [12] to the problem from the theorem. Note that the tree $\left(L(\mathcal{A}) ; \leq_{\text {pref }}\right)$, where $\mathcal{A}$ is a prefix-closed acyclic DFA, is just the unfolding of the underlying dag (directed acyclic graph) in the initial of $\mathcal{A}$. Vice versa, from a dag $D$ with a root node $r$ one can construct a prefix-closed acyclic DFA $\mathcal{A}$ such that $\left(L(\mathcal{A}) ; \leq_{\text {pref }}\right)$ is isomorphic to the unfolding of $D$ in $r$ (let us denote the latter tree by unfold $(D, r)$ ). One only has to associate labels to the edges of $D$. Hence, it suffices to construct from a given monotone circuit $C$ a dag $D$ which contains for every gate $g$ of $C$ two nodes $g_{1}, g_{2}$ such that $g$ evaluates to true if and only if unfold $\left(D, g_{1}\right) \cong$ unfold $\left(D, g_{2}\right)$. This is straightforward for the input gates of $C$. For and- and or-gates of $C$, we can use again the construction of [14]. Take the constructions from Figure 5 and 6 , where in Figure 5 each of the subtrees $[U]_{1},[U]_{2},[V]_{1}$, and $[V]_{2}$ is represented only once. The construction for or-gates is shown in Figure 7. Assume that the dag $D$ below the nodes $u_{1}, u_{2}, v_{1}$, and $v_{2}$ is already constructed. Here $u_{1}$ and $u_{2}$ correspond to a gate $u$ and $v_{1}$ and $v_{2}$ correspond to a gate $v$. Hence, $u$ (resp., $v$ ) evaluates to true if and only if $\operatorname{unfold}\left(D, u_{1}\right) \cong \operatorname{unfold}\left(D, u_{2}\right)\left(\operatorname{resp}\right.$., $\left.\operatorname{unfold}\left(D, u_{1}\right) \cong \operatorname{unfold}\left(D, u_{2}\right)\right)$. Let $t$ be an or-gate with inputs $u$ and $v$. We add the nodes and edges as shown in Figure 7. Then the arguments from the proof of Lemma 3.7 show that $u$ or $v$ evaluates to true if and only if $\operatorname{unfold}\left(D, t_{1}\right) \cong \operatorname{unfold}\left(D, t_{2}\right)$.

\section{Isomorphism problem for regular words}

In this section we study the isomorphism problem for regular words that are represented by partitioned DFAs. We prove that this problem as well as the isomorphism problem for regular linear orders that are represented by DFAs are P-complete. It follows that the isomorphism problem for regular linear orders that are represented by NFAs can be solved in exponential time. We show that this problem is PSPACE-hard. For the case of acyclic DFAs and NFAs, respectively, we obtain completeness results for counting classes ( $\mathrm{C}_{=} \mathrm{L}$-completeness for acyclic DFAs and $\mathrm{C}_{=} \mathrm{P}$-completeness for acyclic NFAs). 


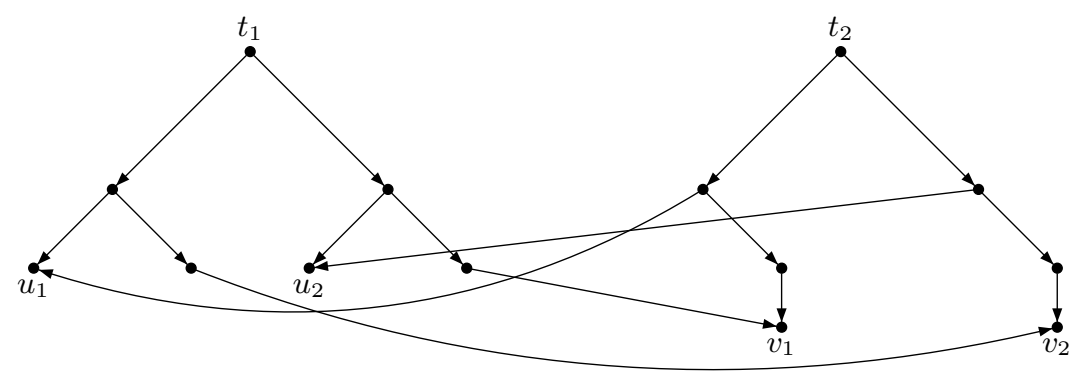

Fig. 7. The or-construction in the proof of Theorem 3.13

\subsection{Upper bounds}

The main result of this section is:

Theorem 4.1. The following problem can be solved in polynomial time:

INPUT: Two partitioned DFAs $\mathcal{A}_{1}$ and $\mathcal{A}_{2}$. QUESTION: $w\left(\mathcal{A}_{1}\right) \cong w\left(\mathcal{A}_{2}\right)$ ?

In Section 4.2-4.6 we prove Theorem 4.15. Section 4.2 will introduce some of the machinery from [2] concerning blocks. Blocks allow to condensate a generalized word to a coarser word (whose elements are the blocks of the original word). In Section 4.3 we will formally introduce succinct regular expressions (expressions in form of dags) and in Section 4.4 we will argue that Heilbrunner's algorithm from [13] allows to transform a given partitioned DFA in polynomial time into an equivalent succinct (regular) expression. Hence, the remaining goal is to develop a polynomial time algorithm for checking whether two given succinct expressions represent isomorphic regular words. For the special case that these regular words consist of only one block (so called primitive regular words), this will be accomplished in Section 4.5. In this step, we will make use of algorithms for straight-line programs (succinctly represented finite words) [27]. Finally, in Section 4.6 we will present a polynomial time algorithm or checking whether two given succinct expressions represent isomorphic regular words.

\subsection{Blocks and their combinatorics}

In this section, we will introduce the crucial notion of a block, and we recall some of the results from [2] that we are using later.

Let $u=(L ; \leq, \tau)$ be a generalized word. An interval of $u$ is an interval of the underlying linear order $(L ; \leq)$. A subword of $u$ is an interval $I$ of $u$ together with the coloring $\tau$ restricted to $I$. Let $\Gamma \subseteq \Sigma$ be finite. A $\Gamma$-uniform subword of $u$ is a subword that is isomorphic to $\Gamma^{\eta}$. A subword is uniform if it is $\Gamma$-uniform for some $\Gamma \subseteq \Sigma$. A uniform subword is a maximal uniform subword if it is not properly contained in another uniform subword. Now let $v$ be a subword such that no point of $v$ is contained in a uniform 
subword of $u$. Then $v$ is successor-closed if for each point $p$ of $v$, whenever the successor and the predecessor of $p$ exist, they are contained in $v$ as well. A successor-closed subword is minimal if it does not strictly contain another successor-closed subword. Following [2] we define:

Definition 4.2 (blocks). Let $u$ be a regular word. A block of $u$ is either a maximal uniform subword of u or a minimal successor-closed subword of $u$.

A regular word which consists of a single block is called primitive. ${ }^{4} \mathrm{By}$ [2] a word $u$ is primitive if and only if it is of one of the following forms (where $x, z \in \Sigma^{+}, y \in \Sigma^{*}$ ): A finite non-empty word, a scattered word of the form $x^{\bar{\omega}} y$, a scattered word of the form $y z^{\omega}$, a scattered word of the form $x^{\bar{\omega}} y z^{\omega}$, or a uniform word ( $\Gamma^{\eta}$ for some $\Gamma \subseteq \Sigma$ ). Let $D(\Sigma)$ be the set of all primitive words over $\Sigma$.

Let $u$ be a regular word. Each point $p$ of $u$ belongs to some unique block $\mathrm{Bl}(p)$, which induces a regular (and hence primitive) word. Moreover we can order the blocks of $u$ linearly by setting $\mathrm{Bl}(p)<\mathrm{Bl}(q)$ if and only if $p<q$. The order obtained that way is denoted $(\mathrm{Bl}(u) ; \leq)$. Then we extend the order $(\mathrm{Bl}(u) ; \leq)$ to a generalized word $\widehat{u}$ over $D(\Sigma)$ (here it is useful to allow infinite alphabets, since $D(\Sigma)$ is infinite), called the skeleton of $u$, by labeling each block with the corresponding isomorphic word in $D(\Sigma)$. Implicitly, it is shown in [2] that for every regular word $u$ there exists a finite subset of $D(\Sigma)$ such that every block of $u$ is isomorphic to a primitive word from that finite subset. Moreover, $\widehat{u}$ is again a regular word. Later it will be convenient to have the following renaming notion available. Let $V$ be a finite alphabet, let $\varphi: V \rightarrow D(\Sigma)$ be an injective mapping and suppose that all blocks of a regular word $u$ belong to the image of $\varphi$. The word $v$ that has $(\operatorname{Bl}(u) ; \leq)$ as underlying order and each block $B$ of $u$ labeled with $\varphi^{-1}(B)$ is called the $\varphi$-skeleton of $u$. We will need the following result from [2]:

Proposition 4.3 (see [2, Corollary 73]). Let $u, v \in \operatorname{Reg}(\Sigma)$. Let $V$ be a finite alphabet and let $\varphi: V \rightarrow D(\Sigma)$ be injective such that all blocks of $u$ and $v$ are in the image of $\varphi$. Then $u$ and $v$ are isomorphic if and only if the $\varphi$-skeletons of $u$ and $v$ are isomorphic.

We will consider finite and infinite sequences, whose symbols are regular words and where the underlying order type is either finite, $\omega$ or $\bar{\omega}$. In the following, when writing $\left(u_{i}\right)_{i \in I}$, we assume that either $I=\{1, \ldots, n\} \neq \emptyset$ (i.e., $\left(u_{i}\right)_{i \in I}$ is the finite sequence $\left.\left(u_{1}, \ldots, u_{n}\right)\right)$ or $I=\{1,2,3, \ldots\}$ (i.e., $\left(u_{i}\right)_{i \in I}$ is the infinite sequence $\left.\left(u_{1}, u_{2}, u_{2}, \ldots\right)\right)$ or $I=\{\ldots,-2,-1,0\}$ (i.e., $\left(u_{i}\right)_{i \in I}$ is the infinite sequence $\left(\ldots, u_{-2}, u_{-1}, u_{0}\right)$ ). The corresponding generalized word is $\prod_{i \in I} u_{i}$ (either $u_{1} \cdots u_{n}$, or $u_{1} u_{2} u_{3} \cdots$ or $\left.\cdots u_{-2} u_{-1} u_{0}\right)$. We say that two sequences $\left(u_{i}\right)_{i \in I}$ and $\left(v_{j}\right)_{j \in J}$ are equivalent, if the generalized words $\prod_{i \in I} u_{i}$ and $\prod_{j \in J} v_{j}$ are isomorphic. We use commas to separate the successive $u_{i}$ in the sequence $\left(u_{i}\right)_{i \in I}$ in order to avoid misinterpretations. For instance $(a, a)$ viewed as a sequence over regular words has length two whereas $(a a)$ has length 1 . Of course, $(a, a)$ and $(a a)$ are equivalent sequences.

\footnotetext{
${ }^{4}$ In combinatorics on words, a finite word is called primitive, if it is not a proper power of a non-empty word. Our notion of a primitive word should not be confused with this definition.
} 
Definition 4.4. Let $\bar{u}=\left(u_{i}\right)_{i \in I}$ be a sequence of regular words. We say that $\bar{u}$ does not merge if the set of blocks of $\prod_{i \in I} u_{i}$ is the union of the set of blocks of the $u_{i}$. If this is not the case, then we say that $\bar{u}$ merges.

In other words, $\bar{u}$ merges if there exists a block that contains elements from two different $u_{i}$. In [2, Corollary 32] it is shown that a sequence $\bar{u}$ merges, if and only if there exists a factor $\left(u_{i}, u_{i+1}\right)$ or $\left(u_{i}, u_{i+1}, u_{i+2}\right)$ that merges.

Example 4.5. Clearly if $u$ and $v$ are finite words, then $(u, v)$ merges. Also, $\left(\Gamma^{\eta}, \Gamma^{\eta}\right)$ and $\left(\Gamma^{\eta}, a, \Gamma^{\eta}\right)$ merge for every $\Gamma \subseteq \Sigma$ and $a \in \Gamma$ (in both cases, the sequence is equivalent to $\left.\Gamma^{\eta}\right)$. On the other hand, $\left([a b]^{\eta},[a b]^{\eta}\right)$ does not merge. The reason is that the blocks of $[a b]^{\eta}$ are the copies of $a b$. More generally, if $u$ is not primitive and $X$ is a finite subset of regular words, then $\left((X \cup\{u\})^{\eta},(X \cup\{u\})^{\eta}\right)$ does not merge.

For the case of a sequence of primitive words, a complete description of merging sequences was given in [2]. Moreover, if a sequence of primitive words merges, then it can be simplified to a non-merging sequence of primitive words. To make this more precise, let $u, v, w$ be primitive words. If $(u, v)$ merges, then by [2, Lemma 24] either $u$ and $v$ are $\Gamma$-uniform for some $\Gamma \subseteq \Sigma$ or $u$ is right-closed and $v$ is left-closed. Then, the regular word $u v$ has a single block. If $(u, v, w)$ merges, then by [2, Lemma 24] either $(u, v)$ merges, or $(v, w)$ merges, or $u, w$ are $\Gamma$-uniform and $v$ is a singleton from $\Gamma$. This motivates the definition of the following rewriting system $R$ over finite sequences over $D(\Sigma)$.

Definition 4.6 (rewriting system $R$ ). The rewriting system $R$ over the set $D(\Sigma)$ consists of the following rules:

$$
\begin{aligned}
& \text { - }\left(u_{1}, u_{2}, u_{3}\right) \rightarrow u \text { if } u_{1}=u_{3}=u=\Gamma^{\eta} \text { for some } \Gamma \subseteq \Sigma \text { and } u_{2} \in \Gamma \\
& \text { - }\left(u_{1}, u_{2}\right) \rightarrow u \text { if one of the following holds: } \\
& \quad \text { - } u_{1} \text { is right-closed, } u_{2} \text { is left-closed and } u=u_{1} u_{2} \\
& \quad \text { - } u_{1}=u_{2}=u=\Gamma^{\eta} \text { for some } \Gamma \subseteq \Sigma .
\end{aligned}
$$

In the following, we will use some basic facts from rewriting theory, see e.g. [4] for further details. For sequences $\bar{x}$ and $\bar{y}$ over $\operatorname{Reg}(\Sigma)$, we write $\bar{x} \rightarrow_{R} \bar{y}$ if there exist a rewrite rule $\bar{u} \rightarrow u$ and an occurrence of the sequence $\bar{u}$ in $\bar{x}$ such that replacing that occurrence by $u$ gives the sequence $\bar{y}$. Here, $\bar{x}$ and $\bar{y}$ may be infinite sequences. Moreover, those $x_{i}$ of $\bar{x}=\left(x_{i}\right)_{i \in I}$ that are not primitive are left untouched in the rewrite step $\bar{x} \rightarrow_{R} \bar{y}$. Clearly, $\bar{x} \rightarrow_{R} \bar{y}$ implies that the sequences $\bar{x}$ and $\bar{y}$ are equivalent. A (possibly infinite) sequence $\bar{u}$ is irreducible w.r.t. $R$ if there does not exist a sequence $\bar{v}$ with $\bar{u} \rightarrow_{R} \bar{v}$. Clearly, on infinite sequences, $R$ cannot be terminating (e.g., $\left(a^{\eta}, a^{\eta}, a^{\eta} \ldots\right) \rightarrow_{R}\left(a^{\eta}, a^{\eta}, a^{\eta} \ldots\right)$ is a loop). On the other hand, $R$ is trivially terminating on finite sequences, since it is length-reducing. Moreover, by analyzing overlapping left-hand sides of $R$, one can easily show:

Lemma 4.7. The rewriting system $R$ is strongly confluent (on finite and infinite sequences), i.e., for all $\bar{u}, \bar{v}, \bar{w}$ such that $\bar{u} \rightarrow_{R} \bar{v}$ and $\bar{u} \rightarrow_{R} \bar{w}$ there exists $\bar{x}$ such that $\left(\bar{v}=\bar{x}\right.$ or $\left.\bar{v} \rightarrow_{R} \bar{x}\right)$ and $\left(\bar{w}=\bar{x}\right.$ or $\left.\bar{w} \rightarrow_{R} \bar{x}\right)$. 
By a simple fact from rewriting theory, it follows that $R$ is also confluent, i.e., for all $\bar{u}, \bar{v}, \bar{w}$ such that $\bar{u} \rightarrow_{R}^{*} \bar{v}$ and $\bar{u} \rightarrow_{R}^{*} \bar{w}$ there exists $\bar{x}$ such that $\bar{v} \rightarrow_{R}^{*} \bar{x}$ and $\bar{w} \rightarrow_{R}^{*} \bar{x}$. Termination (on finite sequences) and confluence imply that $R$ produces unique normal forms for finite sequences, i.e., for every finite sequence $\bar{u}$ there exists a unique finite sequence $\bar{v}$ such that $\bar{u} \rightarrow_{R}^{*} \bar{v}$ and $\bar{v}$ is irreducible w.r.t. $R$. This $\bar{v}$ is called the irreducible normal form of $\bar{u}$.

The following is a direct consequence of [2, Lemma $24 \&$ Corollary 32].

Lemma 4.8. Let $\bar{u}$ be a sequence of primitive words. Then $\bar{u}$ does not merge if and only if $\bar{u}$ is irreducible w.r.t. $R$.

We also have to verify that a sequence $\bar{u}$ over $\operatorname{Reg}(\Sigma)$ containing non-primitive words does not merge. We use the definition below. Note that a regular word need not have a first or last block. For instance, $\left(a^{\omega}\right)^{\omega}$ has a first block but no last block, whereas $\left(a^{\omega}\right)^{\bar{\omega}}\left(a^{\omega}\right)^{\omega}$ and $[a a]^{\eta}$ neither have a first block nor a last block.

Definition 4.9 (good and semi-good sequences). The sequence $\bar{u}=\left(u_{i}\right)_{i \in I}$ is good if the following conditions hold:

(1) $\bar{u}$ is irreducible with respect to $R$.

(2) For all $i \in I$ we have:

(a) If $u_{i}$ is not primitive and has a first block, then either $\left(i-1 \in I, u_{i-1}\right.$ is uniform, and $\left(u_{i-1}, u_{i}\right)$ does not merge) or $\left(i-1, i-2 \in I, u_{i-1}\right.$ and $u_{i-2}$ are primitive, and $\left(u_{i-2}, u_{i-1}, u_{i}\right)$ does not merge).

(b) If $u_{i}$ is not primitive and has a last block, then either $\left(i+1 \in I, u_{i+1}\right.$ is uniform, and $\left(u_{i}, u_{i+1}\right)$ does not merge) or $\left(i+1, i+2 \in I, u_{i+1}\right.$ and $u_{i+2}$ are primitive, and $\left(u_{i}, u_{i+1}, u_{i+2}\right)$ does not merge).

If only (2) holds, then $\bar{u}$ is said to be semi-good.

Lemma 4.10. If $\bar{u}$ is good, then $\bar{u}$ does not merge.

Proof. Assume that $\bar{u}$ is good but merges. By [2, Corollary 32], one of the following cases holds:

Case 1. $\bar{u}$ contains a factor $\left(u_{i}, u_{i+1}\right)$ that merges. If $u_{i}$ and $u_{i+1}$ would be both primitive, then $\bar{u}$ would be not irreducible, which is a contradiction ( $\bar{u}$ is good). Hence, $u_{i}$ or $u_{i+1}$ must be not primitive. W.l.o.g. assume that $u_{i}$ is not primitive (the other case is symmetric). If $u_{i}$ has no last block, then [2, Corollary 30(1)] implies that $\left(u_{i}, u_{i+1}\right)$ does not merge, which is a contradiction. Hence, we can assume that $u_{i}$ has a last block. But then, since $\bar{u}$ is good, $\left(u_{i}, u_{i+1}\right)$ does not merge, which is again a contradiction.

Case 2. $\bar{u}$ contains a factor $\left(u_{i}, u_{i+1}, u_{i+2}\right)$ that merges but neither $\left(u_{i}, u_{i+1}\right)$ nor $\left(u_{i+1}, u_{i+2}\right)$ merges. Since $\bar{u}$ is irreducible w.r.t. $R$, it follows that $u_{i}, u_{i+1}$, or $u_{i+2}$ is not primitive. The case that $u_{i+2}$ is not primitive is symmetric to the case that $u_{i}$ is not primitive. Hence, it suffices to consider the following two subcases:

Case 2a. $u_{i}$ is not primitive. If $u_{i}$ has no last block, then [2, Corollary 31(1)] implies that $\left(u_{i}, u_{i+1}, u_{i+2}\right)$ does not merge, which is a contradiction. Hence, we can assume that $u_{i}$ has a last block, call it $b_{i}$. Since $\bar{u}$ is good and $\left(u_{i}, u_{i+1}, u_{i+2}\right)$ merges, 
$u_{i+1}$ must be uniform. If $u_{i+2}$ has no first block, then again [2, Corollary 31(1)] implies that $\left(u_{i}, u_{i+1}, u_{i+2}\right)$ does not merge, which is a contradiction. Let $b_{i+2}$ be the first block of $u_{i+2}$. Moreover, [2, Corollary 31(2)] implies that $\left(b_{i}, u_{i+1}, b_{i+2}\right)$ merges. Since $\left(u_{i}, u_{i+1}\right)$ and $\left(u_{i+1}, u_{i+2}\right)$ do not merge, also $\left(b_{i}, u_{i+1}\right)$ and $\left(u_{i+1}, b_{i+2}\right)$ do not merge. It follows (from the form of our rewriting system $R$ ) that $b_{i}=b_{i+2}$ is uniform and $u_{i+1}$ is a singleton word. But we have already shown that $u_{i+1}$ is uniform, which is a contradiction.

Case 2b. $u_{i+1}$ is not primitive. Then $u_{i+1}$ has more than one block and [2, Corollary 31(1)] directly implies that $\left(u_{i}, u_{i+1}, u_{i+2}\right)$ does not merge, which is again a contradiction.

Lemma 4.11. If $\bar{u}$ is semi-good and $\bar{u} \rightarrow_{R} \bar{v}$, then $\bar{v}$ is semi-good as well.

Proof. Assume that $\bar{u}=\left(u_{i}\right)_{i \in I}$ is semi-good and $\bar{u} \rightarrow_{R} \bar{v}$. We have to show that $\bar{v}=\left(v_{j}\right)_{j \in J}$ is semi-good. For this, consider an $j \in J$ such that $v_{j}$ is not primitive. Since the system $R$ does not introduce non-primitive words, $v_{j}$ must have been already present in $\bar{u}$. Let $i \in I$ be the position in $\bar{u}$ that corresponds to position $j$ in $\bar{v}$. Hence, $u_{i}=v_{j}$. By symmetry it suffices to show that condition (2a) from Definition 4.9 holds for $j \in J$. The case that $u_{i}=v_{j}$ has no first block is clear. So, assume that $u_{i}$ has a first block. Since $\bar{u}$ is semi-good, we can distinguish the following two cases.

Case 1. $i-1 \in I, u_{i-1}$ is uniform, and $\left(u_{i-1}, u_{i}\right)$ does not merge. From the form of the rewrite rules, it follows that $v_{j-1}=u_{i-1}$. Hence, $v_{j-1}$ is uniform, and $\left(v_{j-1}, v_{j}\right)=$ $\left(u_{i-1}, u_{i}\right)$ does not merge. Thus, we have shown condition (2a) from Definition 4.9 for $j$.

Case 2. $i-1, i-2 \in I, u_{i-2}, u_{i-1}$ are primitive, and $\left(u_{i-2}, u_{i-1}, u_{i}\right)$ does not merge. We make a case distinction on the position, where the rewrite rule is applied.

Case $2 a . i-3 \in I$ and in the rewrite step $\bar{u} \rightarrow_{R} \bar{v},\left(u_{i-3}, u_{i-2}, u_{i-1}\right)$ is replaced by $u \in D(\Sigma)$. Thus, $u_{i-3}=u_{i-1}=u$ is uniform. Hence, $v_{j-1}=u$ is uniform. Moreover, $\left(v_{j-1}, v_{j}\right)=\left(u_{i-1}, u_{i}\right)$ does not merge.

Case 2b. $i-4 \in I$ and in the rewrite step $\bar{u} \rightarrow_{R} \bar{v},\left(u_{i-4}, u_{i-3}, u_{i-2}\right)$ is replaced by $u \in D(\Sigma)$. Thus, $u_{i-4}=u_{i-2}=u$ is uniform, $v_{j-2}=u=u_{i-2}$, and $u_{i-1}=v_{j-1}$. It follows that $v_{j-2}$ and $v_{j-1}$ are primitive, and that $\left(v_{j-2}, v_{j-1}, v_{j}\right)=\left(u_{i-2}, u_{i-1}, u_{i}\right)$ does not merge.

Case $2 c$. In the rewrite step $\bar{u} \rightarrow_{R} \bar{v},\left(u_{i-2}, u_{i-1}\right)$ is replaced by $u \in D(\Sigma)$. Then, $\left(u_{i-2}, u_{i-1}\right)$ merges. But this contradicts the assumption that $\left(u_{i-2}, u_{i-1}, u_{i}\right)$ does not merge.

Case $2 d . i-3 \in I$ and in the rewrite step $\bar{u} \rightarrow_{R} \bar{v},\left(u_{i-3}, u_{i-2}\right)$ is replaced by $u \in D(\Sigma)$. If $u_{i-3}=u_{i-2}=u$ is uniform, then $v_{j-2}=u_{i-2}$ and $v_{j-1}=u_{i-1}$ are primitive and $\left(v_{j-2}, v_{j-1}, v_{j}\right)=\left(u_{i-2}, u_{i-1}, u_{i}\right)$ does not merge. Finally, assume that $u_{i-3}$ is right-closed, $u_{i-2}$ is left-closed and $v_{j-2}=u=u_{i-3} u_{i-2}$. We have $v_{j-1}=$ $u_{i-1}$. Thus $v_{j-1}$ and $v_{j-2}$ are primitive. It remains to show that $\left(v_{j-2}, v_{j-1}, v_{j}\right)=$ $\left(u_{i-3} u_{i-2}, u_{i-1}, u_{i}\right)$ does not merge. We know that $\left(u_{i-1}, u_{i}\right)$ does not merge (since $\left(u_{i-2}, u_{i-1}, u_{i}\right)$ does not merge). Assume that $\left(u_{i-3} u_{i-2}, u_{i-1}\right)$ merges. Then (since 
$u_{i-3} u_{i-2}$ is primitive and scattered and $u_{i-1}$ is primitive) $u_{i-3} u_{i-2}$ must be rightclosed and $u_{i-1}$ must be left-closed. But then, $u_{i-2} \neq \varepsilon$ is right-closed as well and $\left(u_{i-2}, u_{i-1}\right)$ merges. This is a contradiction. Hence, $\left(u_{i-3} u_{i-2}, u_{i-1}\right)$ does not merge. Let $b_{i}$ be the first block of $u_{i}$. If $\left(u_{i-3} u_{i-2}, u_{i-1}, u_{i}\right)$ merges, then by [2, Corollary 31(2)], $\left(u_{i-3} u_{i-2}, u_{i-1}, b_{i}\right)$ merges. Since neither $\left(u_{i-3} u_{i-2}, u_{i-1}\right)$ nor $\left(u_{i-1}, b_{i}\right)$ merges, $u_{i-3} u_{i-2}$ and $b_{i}$ must be uniform. But we know that $u_{i-3} u_{i-2}$ is scattered, which leads again to a contradiction. Thus, indeed $\left(u_{i-3} u_{i-2}, u_{i-1}, u_{i}\right)$ does not merge.

If the rewrite rule is applied at a position different from those considered in Case $2 \mathrm{a}-2 \mathrm{~d}$, then $\left(v_{j-2}, v_{j-1}, v_{j}\right)=\left(u_{i-2}, u_{i-1}, u_{i}\right)$. Since $\left(u_{i-2}, u_{i-1}, u_{i}\right)$ fulfills condition (2a) from Definition 4.9, so does $\left(v_{j-2}, v_{j-1}, v_{j}\right)$. This concludes the proof of the lemma.

Lemma 4.11 implies that from a given finite semi-good sequence $\bar{u}$ we can compute an equivalent good sequence, by computing the (unique) irreducible normal form of $\bar{u}$.

\subsection{Expressions and succinct expressions}

Regular words can be naturally described by expressions using the operations of concatenation, $\omega$-power, $\bar{\omega}$-power, and shuffle. Formally, the set $T(V, \Sigma)$ of expressions over $V$ and $\Sigma$ is inductively defined as follows:

(a) $V \cup \Sigma \subseteq T(V, \Sigma)$

(b) If $\alpha_{1}, \ldots, \alpha_{n} \in T(V, \Sigma)(n \geq 1)$, then $\alpha_{1} \cdots \alpha_{n} \in T(V, \Sigma)$.

(c) If $\alpha \in T(V, \Sigma)$, then $\alpha^{\omega} \in T(V, \Sigma)$ and $\alpha^{\bar{\omega}} \in T(V, \Sigma)$.

(d) If $\alpha_{1}, \ldots, \alpha_{n} \in T(V, \Sigma)(n \geq 1)$, then $\left[\alpha_{1}, \ldots, \alpha_{n}\right]^{\eta} \in T(V, \Sigma)$.

A mapping $f: V \rightarrow \operatorname{Reg}(\Sigma)$ will be extended homomorphically to a mapping $f$ : $T(V, \Sigma) \rightarrow \operatorname{Reg}(\Sigma)$ inductively as follows, where $\alpha, \alpha_{1}, \ldots, \alpha_{n} \in T(V, \Sigma)$ :

- $f(a)=a$ for $a \in \Sigma$

- $f\left(\alpha_{1} \cdots \alpha_{n}\right)=f\left(\alpha_{1}\right) \cdots f\left(\alpha_{n}\right)$

- $f\left(\alpha^{\omega}\right)=f(\alpha)^{\omega}$

- $f\left(\alpha^{\bar{\omega}}\right)=f(\alpha)^{\bar{\omega}}$

- $f\left(\left[\alpha_{1}, \ldots, \alpha_{n}\right]^{\eta}\right)=\left(\left[f\left(\alpha_{1}\right), \ldots, f\left(\alpha_{n}\right)\right]^{\eta}\right.$

For $\alpha \in T(V, \Sigma)$ we define the size $|\alpha| \in \mathbb{N}$ inductively as follows:

- $|\alpha|=1$ for $\alpha \in V \cup \Sigma$

- $\left|\alpha_{1} \cdots \alpha_{n}\right|=\left|\alpha_{1}\right|+\cdots+\left|\alpha_{n}\right|$

- $\left|\alpha^{\omega}\right|=\left|\alpha^{\bar{\omega}}\right|=|\alpha|+1$

- $\left|\left[\alpha_{1}, \ldots, \alpha_{n}\right]^{\eta}\right|=\left|\alpha_{1}\right|+\cdots+\left|\alpha_{n}\right|+1$

A succinct expression system (SES) is a tuple $\mathbb{A}=(V, \Sigma$, rhs) such that:

- $V$ (the set of variables) and $\Sigma$ (the terminal alphabet) are disjoint finite alphabets.

- rhs (for right-hand side) is a mapping from $V$ to $T(V, \Sigma)$ such that the relation $\{(Y, X) \in V \times V \mid Y$ occurs in rhs $(X)\}$ is acyclic. The reflex transitive closure of this relation is called the hierarchical order of $\mathbb{A}$ and denoted by $\preceq_{\mathbb{A}}$. 
The property for rhs ensures that there exists a unique mapping val $\mathbb{A}_{\mathbb{A}}: V \rightarrow \operatorname{Reg}(\Sigma)$ such that val $\left.\right|_{\mathbb{A}}(X)=\operatorname{val}_{\mathbb{A}}(\operatorname{rhs}(X))$ for all $X \in V$. If $\mathbb{A}$ is clear from the context, we will simply write $\operatorname{val}(X)$.

In the following a quadruple $\mathbb{A}=(V, \Sigma$, rhs, $S)$ where $(V, \Sigma$, rhs $)$ is as above and $S \in V$ (i.e., an SES with a distinguished start variable $S$ ) we will be called a succinct expression. In this case let us set val $(\mathbb{A})=\operatorname{val}_{\mathbb{A}}(S)$. A succinct expression may be also seen as a dag (directed acyclic graph), whose unfolding is an expression in the above sense.

Example 4.12. Consider the succinct expression

$$
\mathbb{A}=\left(\left\{X_{1}, X_{2}, X_{3}, X_{4}, X_{5}\right\},\{a, b\}, \text { rhs, } X_{1}\right)
$$

with

$$
\begin{aligned}
& \operatorname{rhs}\left(X_{1}\right)=\left[X_{2}, X_{3}\right]^{\eta} \quad \operatorname{rhs}\left(X_{2}\right)=X_{3} X_{3} \quad \operatorname{rhs}\left(X_{3}\right)=X_{4} X_{4} \\
& \operatorname{rhs}\left(X_{4}\right)=X_{5} X_{6} \quad \operatorname{rhs}\left(X_{5}\right)=a b \quad \operatorname{rhs}\left(X_{6}\right)=b a .
\end{aligned}
$$

We have $\operatorname{val}(\mathbb{A})=[a b b a a b b a, a b b a a b b a a b b a a b b a]^{\eta}$. The corresponding dag looks as follows:

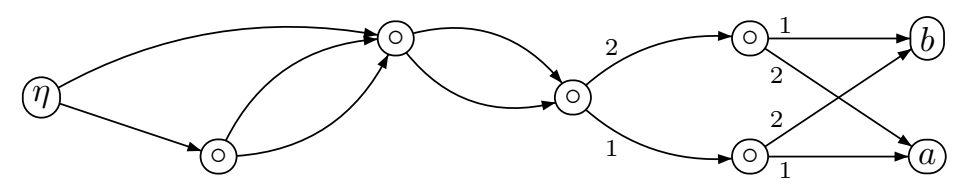

Nodes labelled with $\circ$ compute the concatenation of their successor nodes. In case the order of the successor nodes matters, we specify it by edge labels.

For an SES A we define

$$
|\mathbb{A}|=\sum_{X \in V}|\operatorname{rhs}(X)| .
$$

An SES $\mathbb{A}=(V, \Sigma$, rhs $)$ is in normal form if all right-hand sides are in $(V \cup \Sigma)^{+}$or of the form $Y^{\omega}, Y^{\bar{\omega}},\left[Y_{1}, \ldots, Y_{n}\right]^{\eta}$ for some $Y, Y_{1}, \ldots, Y_{n} \in V \cup \Sigma$. For such an SES $\mathbb{A}$, we define $\operatorname{depth}_{\mathbb{A}}(X)$ and $\omega \eta$-depth $\operatorname{de}_{\mathbb{A}}(X)$ for $X \in V$ inductively as follows (below, we set $\operatorname{depth}_{\mathbb{A}}(a)=\omega \eta$-depth $\operatorname{de}_{\mathbb{A}}(a)=0$ for $\left.a \in \Sigma\right)$ :

- If $\operatorname{rhs}(X)=Y_{1} \cdots Y_{n}\left(n \geq 1, Y_{1}, \ldots, Y_{n} \in \Sigma \cup V\right)$, then

$$
\begin{aligned}
\operatorname{depth}_{\mathbb{A}}(X) & =\max \left(\operatorname{depth}_{\mathbb{A}}\left(Y_{1}\right), \ldots, \operatorname{depth}_{\mathbb{A}}\left(Y_{n}\right)\right)+1, \\
\omega \eta-\operatorname{depth}_{\mathbb{A}}(X) & =\max \left(\omega \eta-\operatorname{depth}_{\mathbb{A}}\left(Y_{1}\right), \ldots, \omega \eta-\operatorname{depth}_{\mathbb{A}}\left(Y_{n}\right)\right) .
\end{aligned}
$$

- If $\operatorname{rhs}(X)=Y^{\omega}$ or $\operatorname{rhs}(X)=Y^{\bar{\omega}}$, then

$$
\begin{aligned}
\operatorname{depth}_{\mathbb{A}}(X) & =\operatorname{depth}_{\mathbb{A}}(Y)+1, \\
\omega \eta-\operatorname{depth}_{\mathbb{A}}(X) & =\omega \eta-\operatorname{depth}_{\mathbb{A}}(Y)+1 .
\end{aligned}
$$

- If $\operatorname{rhs}(X)=\left[Y_{1}, \ldots, Y_{n}\right]^{\eta}$, then

$$
\begin{aligned}
\operatorname{depth}_{\mathbb{A}}(X) & =\max \left(\operatorname{depth}_{\mathbb{A}}\left(Y_{1}\right), \ldots, \operatorname{depth}_{\mathbb{A}}\left(Y_{n}\right)\right)+1 \\
\omega \eta-\operatorname{depth}_{\mathbb{A}}(X) & =\max \left(\omega \eta-\operatorname{depth}_{\mathbb{A}}\left(Y_{1}\right), \ldots, \omega \eta-\operatorname{depth}_{\mathbb{A}}\left(Y_{n}\right)\right)+1 .
\end{aligned}
$$


Straight-line programs. A succinct expression, where all right-hand sides belong to $(V \cup \Sigma)^{+}$is called a straight-line program $(S L P)$ [25]. In this case, $\operatorname{val}(\mathbb{A})$ is a finite non-empty word. An SLP $\mathbb{A}$ can be viewed as a succinct representation of the word $\operatorname{val}(\mathbb{A})$. More precisely, the length of $\operatorname{val}(\mathbb{A})$ may be exponential in $|\mathbb{A}|$. We will make heavy use of the fact that certain algorithmic problems on SLP-encoded finite words can be solved in polynomial time. More precisely, we use the following results:

Remark 4.13. There exist polynomial time algorithms for the following problems:

(a) Given an SLP $\mathbb{A}$, calculate $|\operatorname{val}(\mathbb{A})|$.

(b) Given an SLP $\mathbb{A}$ and a number $k \in \mathbb{N}$ (coded in binary) we can produce an SLP $\mathbb{B}$ of size $|\mathbb{A}|+O(\log k)$ such that $\operatorname{val}(\mathbb{B})=\operatorname{val}(\mathbb{A})^{k}$.

(c) Given an SLP $\mathbb{A}$ and numbers $i \leq j \leq|\operatorname{val}(\mathbb{A})|$, compute an SLP $\mathbb{B}$ with val $(\mathbb{B})=$ $\operatorname{val}(\mathbb{A})[i: j]$. Here $w[i: j]=a_{i} \ldots a_{j}$ for a finite word $w=a_{1} \ldots a_{n}$.

(d) Given SLPs $\mathbb{A}$ and $\mathbb{B}$ decide whether val $(\mathbb{A})=\operatorname{val}(\mathbb{B})[24]$.

(e) Given SLPs $\mathbb{A}$ and $\mathbb{B}$ decide whether val $(\mathbb{A})$ is a factor of val $(\mathbb{B})[11,20,22]$.

The proofs for (a), (b), and (c) are straightforward.

2-level systems. A 2-level system is a tuple $\mathbb{A}=(\mathrm{Up}$, Lo, $\Sigma$, rhs $)$ such that the following holds $\left(f \uparrow_{A}\right.$ denotes the restriction of a function $f$ to the set $A$ ):

- The tuple (Up, Lo, rhs $\left.\Upsilon_{\mathrm{Up}}\right)$ is an SES (w.l.o.g. in normal form) over the terminal alphabet Lo.

- The tuple (Lo, $\Sigma$, rhs $\left.\left.\right|_{\text {Lo }}\right)$ is an SES over the terminal alphabet $\Sigma$.

The set Up (resp. Lo) is called the set of upper level variables (lower level variables) of $\mathbb{A}$. Moreover, we set $V=U \mathrm{p} \cup$ Lo and call it the set of variables of $\mathbb{A}$. The SES (Up, Lo, rhs $\left.\mid U_{\mathrm{p}}\right)$ is called the upper part of $\mathbb{A}$, briefly up $(\mathbb{A})$, and the $\operatorname{SES}\left(\right.$ Lo, $\Sigma$, rhs $\left.\left.\right|_{\text {Lo }}\right)$ is the lower part of $\mathbb{A}$, briefly, lo $(\mathbb{A})$. The upper level evaluation mapping uval $\left.\right|_{\mathbb{A}}: U p \rightarrow$ $\operatorname{Reg}($ Lo $)$ of $\mathbb{A}$ is defined as uval $\left.\right|_{\mathbb{A}}=\operatorname{val}_{\text {up }(\mathbb{A})}$. The evaluation mapping val $\mathbb{A}_{\mathbb{A}}$ is defined by $\operatorname{val}_{\mathbb{A}}(X)=\operatorname{val}_{\mathrm{lo}(\mathbb{A})}\left(\operatorname{val}_{\mathrm{up}(\mathbb{A})}(X)\right)$ for $X \in \mathrm{Up}_{\mathrm{p}}$ and val $\mathrm{A}_{\mathbb{A}}(X)=\operatorname{val}_{\mathrm{lo}(\mathbb{A})}(X)$ for $X \in$ Lo.

\subsection{Heilbrunner's algorithm}

Theorem 4.14. From a given partitioned $D F A \mathcal{A}$, we can compute in polynomial time a succinct expression $\mathbb{A}$ such that $w(\mathcal{A}) \cong \operatorname{val}(\mathbb{A})$.

Proof. There is nothing new about the proof. We just have to follow Heilbrunner's algorithm carefully. Let $\mathcal{A}=\left(Q, \Gamma, \delta, q_{0},\left(F_{a}\right)_{a \in \Sigma}\right)$ be a partitioned DFA and let $F=\bigcup_{a \in \Sigma} F_{a}$. We can assume that every state in $F$ is a dead end, i.e., does not have outgoing transitions. For this, take a new symbol $\$$, as well as a copy $q^{\prime}$ together with the transition $\left(q, \$, q^{\prime}\right)$ for every final state $q \in F$. We set $F_{a}^{\prime}=\left\{q^{\prime} \mid q \in F_{a}\right\}$ and let $\$$ be the smallest symbol in $\Gamma \cup\{\$\}$. The resulting partitioned DFA produces the same generalized word as $\mathcal{A}$.

So, assume that every state in $F$ is a dead end. W.l.o.g. we can also assume that $\mathcal{A}$ is coaccessible. The variables of the succinct expression $\mathbb{A}$ will be the states of $\mathcal{A}$. Consider a state $p \in Q$ and let $\left(p, a_{i}, q_{i}\right)(1 \leq i \leq k)$ be all outgoing transitions for 
$p$, where $a_{1}<a_{2}<\cdots<a_{k}$. Let us define out $(p)=q_{1} q_{2} \cdots q_{k}$. Next, consider the graph with node set $Q$ and an edge from $p \in Q$ to $q \in Q$ if there is a transition from $p$ to $q$. We partition this graph into its strongly connected components (SCCs). An SCC $C$ is smaller than an SCC $D$ if there exists a path from a state in $C$ to a state in $D$; this defines a partial order on the set of SCCs. We eliminate all SCCs starting with the maximal ones. When eliminating an $\operatorname{SCC} C$, we define rhs $_{\mathbb{A}}(p)$ for each state $p \in C$. If the SCC $C$ is a singleton set $\{p\}$ with $p \in F_{a}$, then we set $\operatorname{rhs}_{\mathbb{A}}(p)=a$. If the SCC $C=\{p\}$ is a singleton set with $p \notin F$, then we set $\operatorname{rhs}_{\mathbb{A}}(p)=\operatorname{out}(p)$. Note that out $(p) \neq \varepsilon$, since $p \notin F$ and $\mathcal{A}$ is coaccessible. Now, consider an SCC $C$ of size $|C| \geq 2$. Then every word out $(p)(p \in C)$ contains at least one occurrence of a state from $C$. Hence out $(p)$ can be factored as out $(p)=u_{p} x_{p} v_{p}$, where $u_{p}$ and $v_{p}$ do not contain occurrences of states from the SCC $C$ (i.e., all states occurring in $u_{p}$ and $v_{p}$ belong to larger SCCs), and $x_{p}$ starts and ends with a state from $C$ ( $x_{p}$ might consist of a single state from $C$ ). Define functions $\ell: C \rightarrow C$ and $r: C \rightarrow C$ as follows: $\ell(p)$ (resp. $r(p))$ is the first (resp. last) state of the word $x_{p}$. Then, for every $p \in C$, the sequences $p, \ell(p), \ell^{2}(p), \ldots$ and $p, r(p), r^{2}(p), \ldots$ become periodic after at most $|C|$ steps. We now define regular expressions $\ell_{p}$ and $r_{p}$ as follows: Let $p_{0}, p_{1}, \ldots, p_{a}$ and $q_{0}, q_{1}, \ldots, q_{c}$ be shortest sequences such that $p_{0}=q_{0}=p, p_{i+1}=\ell\left(p_{i}\right), q_{i+1}=r\left(q_{i}\right)$, and $\ell\left(p_{a}\right) \in\left\{p_{0}, p_{1}, \ldots, p_{a}\right\}, r\left(q_{c}\right) \in\left\{q_{0}, q_{1}, \ldots, q_{c}\right\}$. Assume that $\ell\left(p_{a}\right)=p_{b}$ and $r\left(q_{c}\right)=q_{d}$ for $0 \leq b \leq a, 0 \leq d \leq c$. Then, we define

$$
\begin{aligned}
\ell_{p} & =\left(u_{p_{0}} \cdots u_{p_{b-1}}\right)\left(u_{p_{b}} \cdots u_{p_{a}}\right)^{\omega}, \\
r_{p} & =\left(v_{q_{c}} \cdots v_{q_{d}}\right)^{\bar{\omega}}\left(v_{q_{d-1}} \cdots v_{q_{0}}\right) .
\end{aligned}
$$

Next, let $T$ be the set of all regular expressions of the form $\ell_{s} y r_{t}(s, t \in C)$ such that some word out $(p)(p \in C)$ contains a factor $s y t$, where the word $y$ does not contain a state from $C$. Then we finally set $\operatorname{rhs}_{\mathbb{A}}(p)=\ell_{p}[T]^{\eta} r_{p}$ for all $p \in C$. This concludes the elimination step for the SCC $C$. By [13], for every state $p \in Q$ we have $w\left(Q, \Gamma, \delta, p,\left(F_{a}\right)_{a \in \Sigma}\right) \cong \operatorname{val}_{\mathbb{A}}(p)$.

By Theorem 4.14, it suffices to prove the following result in order to prove Theorem 4.1.

Theorem 4.15. The following problem can be solved in polynomial time:

INPUT: Two succinct expressions $\mathbb{A}_{1}$ and $\mathbb{A}_{2}$.

QUESTION: $\operatorname{val}\left(\mathbb{A}_{1}\right) \cong \operatorname{val}\left(\mathbb{A}_{2}\right)$ ?

In the next section, we will prove this result for the special case that both $\operatorname{val}\left(\mathbb{A}_{1}\right)$ and $\operatorname{val}\left(\mathbb{A}_{2}\right)$ are primitive.

\subsection{A polynomial time equivalence test for succinct primitive expressions}

By Theorem 4.14, the remaining goal is to test in polynomial time, whether two succinct expressions represent isomorphic regular words. In a first step, we accomplish this for succinct expressions that represent primitive words. In the following, $\Sigma$ will always refer to a finite alphabet. Let us first show that we can decide in polynomial time whether a succinct expression represents a primitive word. 


\begin{tabular}{|c|c|c|c|c|c|c|}
\hline \multicolumn{2}{|c|}{$u_{1}$} & \multicolumn{1}{|c|}{$u_{1}$} & \multicolumn{2}{|c|}{$v_{1}$} & $w_{1}$ & $w_{1}$ \\
\hline
\end{tabular}

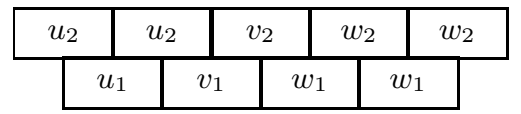

Fig. 8.

Lemma 4.16. Given a succinct expression $\mathbb{A}$, we can decide in polynomial time whether $\operatorname{val}(\mathbb{A})$ is a primitive word, and in case it is we can compute in polynomial time a representation, which has one of the following forms, where $\mathbb{B}, \mathbb{C}, \mathbb{D}$ are SLPs and $\Gamma \subseteq \Sigma$ (here, we should allow also the empty word for $\operatorname{val}(\mathbb{C})$ ): $\operatorname{val}(\mathbb{B})$, $\operatorname{val}(\mathbb{C}) \operatorname{val}(\mathbb{D})^{\omega}$, $\operatorname{val}(\mathbb{B})^{\bar{\omega}} \operatorname{val}(\mathbb{C}), \operatorname{val}(\mathbb{B})^{\bar{\omega}} \operatorname{val}(\mathbb{C}) \operatorname{val}(\mathbb{D})^{\omega}, \Gamma^{\eta}$

Proof. We proceed along the hierarchical order of $\mathbb{A}$ and compute for each variable $A$ of $\mathbb{A}$ whether $\operatorname{val}(A)$ is of one of the following forms $\left(u, w \in \Sigma^{+}, v \in \Sigma^{*}, \Gamma \subseteq \Sigma\right.$, $a, b \in \Gamma): v, u^{\bar{\omega}} v, v w^{\omega}, u^{\bar{\omega}} v w^{\omega}, \Gamma^{\eta}, a \Gamma^{\eta}, \Gamma^{\eta} b, a \Gamma^{\eta} b$. Moreover, SLPs for the finite words $u, v$, and $w$ can computed simultaneously. Observe that from $\operatorname{rhs}(A)$ and the information already computed we can easily obtain whether val $(A)$ is of such a form and in this case of which form. The following identities have to be used for shuffles $\left(\Gamma \subseteq \Sigma, n \geq 0, m \geq 1, a, a_{1}, \ldots, a_{n} \in \Gamma\right.$, and every $u_{i}(1 \leq i \leq m)$ has one of the forms $\Gamma^{\eta}, c \Gamma^{\eta}, \Gamma^{\eta} c, c \Gamma^{\eta} d$ with $c, d \in \Gamma$ )

$$
\begin{gathered}
{\left[a_{1}, \ldots, a_{n}, u_{1}, \ldots, u_{m}\right]^{\eta} \cong \Gamma^{\eta}} \\
\Gamma^{\eta} \Gamma^{\eta} \cong \Gamma^{\eta} a \Gamma^{\eta} \cong\left(\Gamma^{\eta}\right)^{\omega} \cong\left(\Gamma^{\eta}\right)^{\bar{\omega}} \cong\left(\Gamma^{\eta} a\right)^{\omega} \cong\left(a \Gamma^{\eta}\right)^{\bar{\omega}} \cong \Gamma^{\eta} \\
\left(a \Gamma^{\eta}\right)^{\omega} \cong a \Gamma^{\eta} \\
\left(\Gamma^{\eta} a\right)^{\bar{\omega}} \cong \Gamma^{\eta} a
\end{gathered}
$$

All these identities can be deduced from the axioms for regular expressions in [2]. Now $\operatorname{val}(\mathbb{A})$ is primitive if and only if $\operatorname{val}(S)$ is of one of the following forms $(u, w \in$ $\left.\Sigma^{+}, v \in \Sigma^{*}, \Gamma \subseteq \Sigma\right): v, u^{\bar{\omega}} v, v w^{\omega}, u^{\bar{\omega}} v w^{\omega}, \Gamma^{\eta}$.

For our polynomial time equivalence test for succinct expressions that represent primitive words, we need the following technical lemma.

Lemma 4.17. Let $u_{i}, v_{i}, w_{i}(i \in\{1,2\})$ be finite words such that $\left|u_{1}\right|=\left|u_{2}\right|=\left|v_{1}\right|=$ $\left|v_{2}\right|=\left|w_{1}\right|=\left|w_{2}\right|>0$. Then $u_{1}^{\bar{\omega}} v_{1} w_{1}^{\omega}=u_{2}^{\bar{\omega}} v_{2} w_{2}^{\omega}$ if and only if one of the following conditions hold:

- $u_{2} v_{2} w_{2}^{2}$ is a factor of $u_{1}^{2} v_{1} w_{1}^{2}$.

- $u_{1} v_{1} w_{1}^{2}$ is a factor of $u_{2}^{2} v_{2} w_{2}^{2}$.

- $v_{1}=w_{1}, u_{2}=v_{2}$, and $u_{2} w_{2}^{2}$ is a factor of $u_{1}^{2} w_{1}^{2}$.

- $u_{1}=v_{1}, v_{2}=w_{2}$, and $u_{1} w_{1}^{2}$ is a factor of $u_{2}^{2} w_{2}^{2}$.

Proof. The four conditions from the lemma are shown in Figure 8 and Figure 9. It is straightforward to show that any of these four situations implies $u_{1}^{\bar{\omega}} v_{1} w_{1}^{\omega}=u_{2}^{\bar{\omega}} v_{2} w_{2}^{\omega}$. 


\begin{tabular}{|c|c|c|c|c|}
\hline$u_{1}$ & $u_{1}$ & $w_{1}$ & & $w$ \\
\hline & & & $w$ & \\
\hline
\end{tabular}

\begin{tabular}{|c|c|c|c|}
\hline \multicolumn{1}{|c|}{$u_{1}$} & \multicolumn{2}{|c|}{$w_{1}$} & \multicolumn{2}{c|}{$w_{1}$} \\
\hline$u_{2}$ & $u_{2}$ & $w_{2}$ & $w_{2}$ \\
\hline
\end{tabular}

Fig. 9.

For instance, if the left situation in Figure 8 occurs, then there exist words $x, y, x^{\prime}, y^{\prime}$ such that $u_{1}=x y, u_{2}=y x, w_{1}=x^{\prime} y^{\prime}, w_{2}=y^{\prime} x^{\prime}$ and $v_{2} w_{2}=y v_{1} x^{\prime}$. Hence,

$$
u_{1}^{\bar{\omega}} v_{1} w_{1}^{\omega}=(x y)^{\bar{\omega}} v_{1}\left(x^{\prime} y^{\prime}\right)^{\bar{\omega}}=(y x)^{\bar{\omega}} y v_{1} x^{\prime}\left(y^{\prime} x^{\prime}\right)^{\bar{\omega}}=u_{2}^{\bar{\omega}} v_{2} w_{2} w_{2}^{\bar{\omega}}=u_{2}^{\bar{\omega}} v_{2} w_{2}^{\omega}
$$

Let us now assume that $u_{1}^{\bar{\omega}} v_{1} w_{1}^{\omega}=u_{2}^{\bar{\omega}} v_{2} w_{2}^{\omega}$. We distinguish the following cases:

Case 1. The occurrence of $v_{1}$ in $u_{1}^{\bar{\omega}} v_{1} w_{1}^{\omega}$ overlaps the occurrence of $v_{2}$ in $u_{2}^{\bar{\omega}} v_{2} w_{2}^{\omega}$. Then, either $u_{2} v_{2} w_{2}^{2}$ is a factor of $u_{1}^{2} v_{1} w_{1}^{2}$ (if $v_{2}$ starts before $v_{1}$ ) or $u_{1} v_{1} w_{1}^{2}$ is a factor of $u_{2}^{2} v_{2} w_{2}^{2}$ (if $v_{1}$ starts before $v_{2}$ ), see Figure 8 .

Case 2. The occurrence of $v_{1}$ in $u_{1}^{\bar{\omega}} v_{1} w_{1}^{\omega}$ does not overlap the occurrence of $v_{2}$ in $u_{2}^{\bar{\omega}} v_{2} w_{2}^{\omega}$

Case 2.1. The occurrence of $u_{1} v_{1} w_{1}$ in $u_{1}^{\bar{\omega}} v_{1} w_{1}^{\omega}$ overlaps the occurrence of $v_{2}$ in $u_{2}^{\bar{\omega}} v_{2} w_{2}^{\omega}$. Then, one of the following two situations occurs:

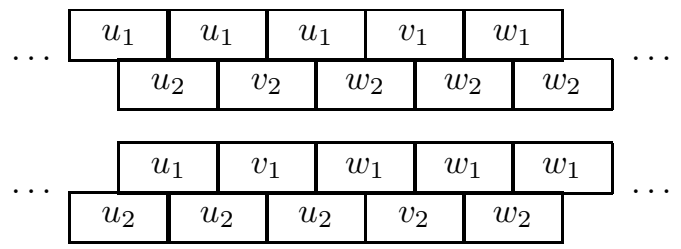

In the first situation, we obtain $v_{1}=w_{1}$ (since $v_{1} w_{1}$ is a factor of $w_{2}^{3}$ ) and $u_{2}=v_{2}$ (since $u_{2} v_{2}$ is a factor of $u_{1}^{3}$ ). Hence, we get the left situation shown in Figure 9, i.e., $u_{2} w_{2}^{2}$ is a factor of $u_{1}^{2} w_{1}^{2}$. In the second situation, we obtain $u_{1}=v_{1}$ (since $u_{1} v_{1}$ is a factor of $u_{2}^{3}$ ) and $v_{2}=w_{2}$ (since $v_{2} w_{2}$ is a factor of $w_{1}^{3}$ ). Hence, we get the right situation shown in Figure 9, i.e., $u_{1} w_{1}^{2}$ is a factor of $u_{2}^{2} w_{2}^{2}$.

Case 2.2. The occurrence of $u_{1} v_{1} w_{1}$ in $u_{1}^{\bar{\omega}} v_{1} w_{1}^{\omega}$ does not overlap the occurrence of $v_{2}$ in $u_{2}^{\bar{\omega}} v_{2} w_{2}^{\omega}$. Then $u_{1} v_{1} w_{1}$ either occurs in $u_{2}^{\bar{\omega}}$ or $w_{2}^{\omega}$. Hence, $u_{1}=v_{1}=w_{1}$ and similarly $u_{2}=v_{2}=w_{2}$. But $u_{1}^{\bar{\omega}} u_{1}^{\omega}=u_{2}^{\bar{\omega}} u_{2}^{\omega}$ implies that $u_{2}^{3}$ is a factor of $u_{1}^{4}$. Hence, the third condition from the lemma holds.

Lemma 4.18. Given two succinct expressions $\mathbb{A}_{1}, \mathbb{A}_{2}$ over $\Sigma$ such that $\operatorname{val}\left(\mathbb{A}_{1}\right)$ and $\operatorname{val}\left(\mathbb{A}_{2}\right)$ are primitive words, we can decide in polynomial time whether $\operatorname{val}\left(\mathbb{A}_{1}\right)=$ $\operatorname{val}\left(\mathbb{A}_{2}\right)$.

Proof. We have to distinguish the following cases:

Case 1. $\operatorname{val}\left(\mathbb{A}_{i}\right)(i \in\{1,2\})$ is finite. Then $\operatorname{val}\left(\mathbb{A}_{1}\right)=\operatorname{val}\left(\mathbb{A}_{2}\right)$ can be checked in polynomial time by Remark 4.13(d). 
Case 2. $\operatorname{val}\left(\mathbb{A}_{i}\right)$ is $\Gamma_{i}$-uniform $(i \in\{1,2\})$. Then $\operatorname{val}\left(\mathbb{A}_{1}\right)=\operatorname{val}\left(\mathbb{A}_{2}\right)$ if and only if $\Gamma_{1}=\Gamma_{2}$ which can be checked in polynomial time.

Case 3. $\operatorname{val}\left(\mathbb{A}_{i}\right)=u_{i} v_{i}^{\omega}(i \in\{1,2\})$. By Lemma 4.16 we can produce SLPs for $u_{i}$ and $v_{i}(i \in\{1,2\})$ from $\mathbb{A}_{1}$ and $\mathbb{A}_{2}$, respectively, in polynomial time. Let $k_{i}=\left|u_{i}\right|$ and $\ell_{i}=$ $\left|v_{i}\right|$. Let $\operatorname{gcm}\left(\ell_{1}, \ell_{2}\right)$ denote the greatest common multiple of $\ell_{1}$ and $\ell_{2}$. By replacing $v_{i}$ by $v_{i}^{\max \left(k_{1}, k_{2}\right) \cdot \operatorname{gcm}\left(\ell_{1}, \ell_{2}\right) / \ell_{i}}$ (for which we can compute an SLP in polynomial time by Remark 4.13(b)), we can assume that $\left|v_{1}\right|=\left|v_{2}\right| \geq k_{1}, k_{2}$. Let $\ell=\left|v_{1}\right|=\left|v_{2}\right|$. W.l.o.g assume that $k_{1} \leq k_{2}$ and let $k=k_{2}-k_{1} \leq \ell$. Then, we can replace $u_{1}$ and $v_{1}$ by $u_{1} v_{1}[1: k]$ and $v_{1}[k+1: \ell] v_{1}[1: k]$, respectively (we can compute SLPs for these words in polynomial time by Remark 4.13(c)). Hence, we can also assume that $\left|u_{1}\right|=\left|u_{2}\right|$. But then, $u_{1} v_{1}^{\omega}=u_{2} v_{2}^{\omega}$ if and only if $u_{1}=u_{2}$ and $v_{1}=v_{2}$, which can be checked in polynomial time by Remark 4.13(d).

Case 4. $\operatorname{val}\left(\mathbb{A}_{i}\right)=u_{i}^{\bar{\omega}} v_{i}(i \in\{1,2\})$. This case can be dealt with analogously to Case 3 .

Case 5. $\operatorname{val}\left(\mathbb{A}_{i}\right)=u_{i}^{\bar{\omega}} v_{i} w_{i}^{\omega}(i \in\{1,2\})$. By Lemma 4.16 we can produce SLPs for $u_{i}, v_{i}$, and $w_{i}$ in polynomial time. As in Case 3 , by replacing the words $u_{i}, w_{i}$ by appropriate powers, we can enforce the condition $\left|u_{1}\right|=\left|u_{2}\right|=\left|w_{1}\right|=\left|w_{2}\right|=\ell \geq$ $\left|v_{1}\right|,\left|v_{2}\right|$. In addition, we can enforce the condition $\left|v_{1}\right|=\left|v_{2}\right|=\ell$ as follows: Let $k_{i}=\left|v_{i}\right| \leq \ell$. Then we can replace $v_{i}$ and $w_{i}$ by $v_{i} w_{i}\left[1: \ell-k_{i}\right]$ and $w_{i}\left[\ell-k_{i}+1\right.$ : $\ell] w_{i}\left[1: \ell-k_{i}\right]$, respectively. Now, that we have $\left|u_{1}\right|=\left|u_{2}\right|=\left|v_{1}\right|=\left|v_{2}\right|=\left|w_{1}\right|=$ $\left|w_{2}\right|$, we can check $u_{1}^{\bar{\omega}} v_{1} w_{1}^{\omega}=u_{2}^{\bar{\omega}} v_{2} w_{2}^{\omega}$ in polynomial time using Lemma 4.17 and Remark 4.13(e).

\subsection{A polynomial time equivalence test for succinct expressions}

In this section, we will finally prove Theorem 4.15 . The general strategy is very similar to [2]. We will incrementally reduce the $\omega \eta$-depth of the two given succinct expressions, until one of them (or both) describe primitive words. This allows to use the results from the previous section. We have to analyze carefully the size of the intermediate succinct expressions. In the following, $\Sigma$ will always refer to a finite alphabet. We will need certain nice properties of SESs.

Definition 4.19 (primitive). A primitive SES is an $S E S \mathbb{A}=(V, \Sigma$, rhs) such that $\operatorname{val}_{\mathbb{A}}(X)$ is primitive for all $X \in V$. A 2-level system $\mathbb{B}$ is primitive if $\mathrm{lo}(\mathbb{B})$ is primitive.

Definition 4.20 (irredundant). An irredundant SES is an $S E S \mathbb{A}=(V, \Sigma$, rhs) such that $\operatorname{val}_{\mathbb{A}}(X) \neq \operatorname{val}_{\mathbb{A}}(Y)$ for all $X, Y \in V$ with $X \neq Y$. Again we say that a 2-level system $\mathbb{B}$ is irredundant if $\mathrm{lo}(\mathbb{B})$ is irredundant.

One can think of a primitive and irredundant SES as a succinct representation of a finite subset of $D(\Sigma)$ where val $\mathrm{A}_{\mathbb{A}}: V \rightarrow D(\Sigma)$ defines an injective mapping from $V$ to this finite subset. Hence, for a regular word $u$ such that all blocks belong to the image of $\left.v\right|_{\mathbb{A}}$, we can define the $\left.v^{2}\right|_{\mathbb{A}}$-skeleton of $u$. In the following, we will simply call it the $\mathbb{A}$-skeleton of $u$. A primitive and irredundant 2-level system intuitively is a system, where the terminal alphabet is a finite subset of $D(\Sigma)$ (namely the valuations of the variables of the lower part lo $(\mathbb{B})$ ). 
Remark 4.21. If a primitive 2-level system $\mathbb{B}$ is not irredundant then, using Lemma 4.18, one can produce in polynomial time an irredundant 2-level system $\mathbb{C}$ such that val $(\mathbb{B})=$ $\operatorname{val}(\mathbb{C})$. Indeed, if there are two different variables $X, Y \in$ Lo such that $\operatorname{val}_{\mathbb{B}}(X)=$ $\operatorname{val}_{\mathbb{A}}(Y)$, then one has to replace $X$ in all right-hand sides by $Y$. Thereafter $X$ can be removed from $L o$. Note that this process does not change the set of upper level variables of $\mathbb{B}$.

Assume that $\mathbb{B}$ is an SES or 2-level system and let $u=\left(A_{i}\right)_{i \in I}$ be a (possibly infinite) sequence of variables of $\mathbb{B}$. We say that $u$ does not merge (is good, semi-good, irreducible), if the sequence $\left(\operatorname{val}\left(A_{i}\right)\right)_{i \in I}$ does not merge (is good, semi-good, irreducible). Moreover, two sequences $u=\left(A_{i}\right)_{i \in I}$ and $v=\left(B_{j}\right)_{j \in J}$ of variables (possibly from two different SESs or 2-level systems) are equivalent if the sequences $\left(\operatorname{val}\left(A_{i}\right)\right)_{i \in I}$ and $\left(\operatorname{val}\left(B_{j}\right)\right)_{j \in J}$ are equivalent (i.e., $\prod_{i \in I} \operatorname{val}\left(A_{i}\right)$ and $\prod_{j \in J} \operatorname{val}\left(B_{j}\right)$ are isomorphic generalized words). The following definition is an adaption of the definition of a proper expression in [2].

Definition 4.22 (proper). Let $\mathbb{B}=(\mathrm{Up}, \mathrm{Lo}, \Sigma$, rhs) be a primitive 2-level system. $A$ variable $X \in \mathrm{Lo} \cup \cup \mathrm{p}$ is proper if one of the following cases holds:

(1) $X \in$ Lo

(2) $\operatorname{rhs}(X)=Y_{1} \cdots Y_{n}$, where $Y_{1} \cdots Y_{n}$ does not merge and $Y_{1}, \ldots, Y_{n}$ are proper.

(3) $\operatorname{rhs}(X)=Y^{\omega}$ or $\operatorname{rhs}(X)=Y^{\bar{\omega}}$, where $Y$ is proper and $Y Y Y$ does not merge.

(4) $\operatorname{rhs}(X)=\left[Y_{1}, \ldots, Y_{n}\right]^{\eta}$ where $Y_{1}, \ldots, Y_{n}$ are proper and $\operatorname{val}(X)$ is not primitive.

The 2-level system $\mathbb{B}$ is proper if $\mathbb{B}$ is irredundant, primitive, and all variables are proper.

Note that the condition that $Y Y Y$ does not merge in Definition 4.22(3) implies that $Y Y Y \cdots$ and $\cdots Y Y Y$ both do not merge by [2, Corollary 32]. Moreover, condition (4) from Definition 4.22 means that $Y_{1}, \ldots, Y_{n}$ are proper and at least on $\operatorname{val}\left(Y_{i}\right)$ is not a single symbol.

Lemma 4.23 (see [2, Corollary 75]). Let $\mathbb{B}$ be a proper 2-level system and $X$ an upper level variable. Then $\mathrm{uval}(X)$ is the lo $(\mathbb{B})$-skeleton of $\mathrm{val}(X)$.

The next two lemmas will be used to make a given 2-level system proper.

Lemma 4.24. Given a primitive 2-level system $\mathbb{B}$ and a finite semi-good sequence $A_{1} \cdots A_{m}$ of variables of $\mathbb{B}$, we can produce in polynomial time a primitive 2-level system $\mathbb{C}$ and a sequence $B_{1} \cdots B_{n}$ of variables of $\mathbb{C}$ such that the following holds:

- The upper parts of $\mathbb{B}$ and $\mathbb{C}$ are the same, and the lower part of $\mathbb{C}$ extends the lower part of $\mathbb{B}$ by at most $m-1$ many new lower level variables, whose right-hand sides have length 2.

- The sequence $B_{1} \cdots B_{n}$ is good.

- $A_{1} \cdots A_{m}$ and $B_{1} \cdots B_{n}$ are equivalent sequences.

- The subsequence of upper level variables in $A_{1} \cdots A_{m}$ is the same as the subsequence of upper level variables in $B_{1} \cdots B_{n}$.

$-n \leq m$. 
Proof. As long as the sequence $A_{1} \cdots A_{m}$ contains a factor $A_{i} A_{i+1}$ or $A_{i} A_{i+1} A_{i+2}$, whose evaluation is a left-hand side of our rewriting system $R$, we do the following:

If $\operatorname{val}\left(A_{i}\right)$ is right-closed and $\operatorname{val}\left(A_{i+1}\right)$ is left-closed, then we introduce a new lower level variable $A$, set $\operatorname{rhs}(A)=A_{i} A_{i+1}$, and replace the sequence $A_{1} \cdots A_{m}$ by the sequence $A_{1} \cdots A_{i-1} A A_{i+2} \cdots A_{m}$. If $\operatorname{val}\left(A_{i}\right)=\operatorname{val}\left(A_{i+1}\right)=\Gamma^{\eta}$ for some $\Gamma \subseteq \Sigma$, we continue with the sequence $A_{1} \cdots A_{i-1} A_{i+1} \cdots A_{m}$. Finally, if val $\left(A_{i}\right)=$ $\operatorname{val}\left(A_{i+2}\right)=\Gamma^{\eta}$ for some $\Gamma \subseteq \Sigma$ and $\operatorname{val}\left(A_{i+1}\right)=a \in \Gamma$, we continue with the sequence $A_{1} \cdots A_{i-1} A_{i+2} \cdots A_{m}$. We iterate this process as long as possible.

Lemma 4.25. Given a primitive 2-level system $\mathbb{B}$ and a finite irreducible sequence $A_{1} \cdots A_{k}(k \geq 3)$, where every $A_{i}$ is a lower level variable of $\mathbb{B}$, we can produce in polynomial time a primitive 2-level SES $\mathbb{C}$ and sequences $B_{1} \cdots B_{m}, C_{1} \cdots C_{n}(m \geq 0$, $n \geq 1$ ) of lower level variables of $\mathbb{C}$ such that the following holds:

- The upper parts of $\mathbb{B}$ and $\mathbb{C}$ are the same, and the lower part of $\mathbb{C}$ extends the lower part of $\mathbb{B}$ by at most one new lower level variable, whose right-hand side has length 2.

- The infinite sequence $B_{1} \cdots B_{m}\left(C_{1} \ldots C_{n}\right)^{\omega}$ is irreducible.

- $\left(A_{1} \cdots A_{k}\right)^{\omega}$ and $B_{1} \cdots B_{m}\left(C_{1} \cdots C_{n}\right)^{\omega}$ are equivalent sequences.

- $m, n \leq k$.

Proof. W.l.o.g. assume that $\left(A_{1} \cdots A_{k}\right)^{\omega}$ is not irreducible. Since $A_{1} \cdots A_{k}$ is irreducible, an $R$-reduction in the infinite sequence $A_{1} \cdots A_{k} A_{1} \cdots A_{k} A_{1} \cdots A_{k} \cdots$ can only occur at a border between $A_{k}$ and $A_{1}$. There are the following cases, according to the left-hand sides of the system $R$.

Case 1. val $\left(A_{k}\right)=\operatorname{val}\left(A_{1}\right)=\Gamma^{\eta}$ for some $\Gamma \subseteq \Sigma$. Then, the infinite sequence $A_{1} A_{2} \cdots A_{k}\left(A_{2} \cdots A_{k}\right)^{\omega}$ is irreducible and equivalent to our original sequence (recall that $k \geq 3)$.

Case 2. val $\left(A_{k}\right)$ is scattered and right-closed, $\operatorname{val}\left(A_{1}\right)$ is scattered and left-closed. Then, we introduce a new lower level variable $A$ with $\operatorname{rhs}(A)=A_{k} A_{1}$. It follows that the infinite sequence $A_{1} A_{2} \cdots A_{k-1}\left(A A_{2} \cdots A_{k-1}\right)^{\omega}$ is irreducible and equivalent to our original sequence.

Case 3. val $\left(A_{k}\right)=\Gamma^{\eta}, \operatorname{val}\left(A_{1}\right)=a, \operatorname{val}\left(A_{2}\right)=\Gamma^{\eta}$ for some $\Gamma \subseteq \Sigma$ and $a \in \Gamma$. If $k=$ 3 , then $A_{1} A_{2} \cdots A_{k}=A_{1} A_{2} A_{3}$ would not be irreducible (since val $\left(A_{2}\right)=\operatorname{val}\left(A_{3}\right)=$ $\left.\Gamma^{\eta}\right)$, which contradicts our assumptions. Hence, assume that $k \geq 4$. Then, the sequence $A_{1} A_{2} \cdots A_{k}\left(A_{3} \cdots A_{k}\right)^{\omega}$ is again irreducible and equivalent to our original sequence.

Case 4. $\operatorname{val}\left(A_{k-1}\right)=\Gamma^{\eta}, \operatorname{val}\left(A_{k}\right)=a, \operatorname{val}\left(A_{1}\right)=\Gamma^{\eta}$ for some $\Gamma \subseteq \Sigma$ and $a \in \Gamma$. This case is similar to Case 3 .

Let $\mathbb{B}$ be an SES and $X$ a variable with $\omega \eta$-depth $(X)=h \geq 1$. Then there is a sequence of variables $X_{1}, \ldots, X_{h}$ such that $X_{h}=X, X_{i} \preceq_{\mathbb{B}} X_{i+1}$, and $\omega \eta-\operatorname{depth}\left(X_{i}\right)=$ $i$. Note that $\operatorname{val}\left(X_{1}\right)$ is either primitive or a shuffle of finite words. If $\operatorname{val}\left(X_{1}\right)=$ $\left[u_{1}, \ldots, u_{k}\right]^{\eta}$ where at least one of the $u_{i}$ is in $\Sigma^{\geq 2}$ (thus, $\operatorname{val}\left(X_{1}\right)$ is not primitive), then this sequence is called a bad sequence. If a variable $X$ has a bad sequence, then we say it is of bad shape. Otherwise it is of good shape. For instance, if $\operatorname{rhs}(X)=[Y]^{\eta}$ and $\operatorname{rhs}(Y)=a b$, then $X$ is of bad shape. 
Proposition 4.26. Let $\mathbb{B}=(V, \Sigma$, rhs) be an SES such that for every variable $X \in V$, either $\operatorname{rhs}(X) \in \Sigma^{+} \cup \Sigma^{*} V \Sigma^{*} \cup V V$ or $\operatorname{rhs}(X)$ is of the form $Y^{\omega}, Y^{\bar{\omega}}$, or $\left[Y_{1}, \ldots, Y_{n}\right]^{\eta}$ for $Y, Y_{1}, \ldots, Y_{n} \in V \cup \Sigma$. Given $\mathbb{B}$ we can produce in polynomial time a proper 2level system $\mathbb{C}=(\mathrm{Up}, \mathrm{Lo}, \Gamma$, rhs $)$ such that every variable $X \in V$, where $\operatorname{val}_{\mathbb{B}}(X)$ is not primitive, belongs to Up and for each of these variables $X$ we have:

(a) $\operatorname{val}_{\mathbb{B}}(X)=\operatorname{val}_{\mathbb{C}}(X)$

(b) If $X$ is of good shape in $\mathbb{B}$, then $\omega \eta$-depth $\mathbb{B}(X)>\omega \eta$-depth $\operatorname{dp}_{(\mathbb{C})}(X)$.

(c) If $X$ is of bad shape in $\mathbb{B}$, then $\omega \eta$-depth $\operatorname{di}_{\mathbb{B}}(X)=\omega \eta$-depth $\operatorname{up}_{(\mathbb{C})}(X)$ and $X$ is of good shape in up $(\mathbb{C})$.

Proof. W.l.o.g. we can assume that $\operatorname{val}(\mathbb{B})$ is not primitive. We start with some preprocessing.

Preprocessing. First we transform our succinct expression $\mathbb{B}$ into a 2-level system $\mathbb{C}$ by collecting in Lo all variables $X$ such that $\operatorname{val}(X)$ is primitive. This can be done in polynomial time using Lemma 4.16. Note that if $\mathrm{val}(X)$ is primitive and scattered, then for every $Y$ in $\operatorname{rhs}(X), \operatorname{val}(Y)$ is primitive too. $\operatorname{But}$ if $\operatorname{val}(X)$ is primitive and dense (i.e., of the form $\Gamma^{\eta}$ for some $\left.\Gamma \subseteq \Sigma\right)$, then this is not necessarily true. ${ }^{5}$ Hence, in this case we have to redefine $\operatorname{rhs}(Y)=\Gamma^{\eta}$. After this process the 2-level system $\mathbb{C}$ is already primitive, satisfies conditions (a), (b), and (c) in our proposition, and for all $X \in \mathrm{Up}$ the word $\operatorname{val}(X)$ is not primitive. All these properties will stay invariant throughout the remaining proof where we manipulate the system $\mathbb{C}$ in order to make it proper.

Before we come to the actual algorithm we transform $\mathbb{C}$ for technical convenience such that for all $X \in \mathrm{Up}$ one of the following holds:

(1) $\operatorname{rhs}(X) \in \mathrm{Lo}^{\geq 2} \cup \mathrm{Lo}^{*} \mathrm{UpLo}^{*}$,

(2) $\operatorname{rhs}(X)=\left[Y_{1}, \ldots Y_{n}\right]^{\eta}$ for some $Y_{1}, \ldots, Y_{n} \in \mathrm{Up} \cup \mathrm{Lo}$,

(3) $\operatorname{rhs}(X) \in U$ Upup,

(4) $\operatorname{rhs}(X)=Y^{\omega}$ for $Y \in U p \cup$ Lo,

(5) $\operatorname{rhs}(X)=Y^{\bar{\omega}}$ for $Y \in$ Up $\cup$ Lo.

In order to achieve this form we simply introduce for each upper level variable $X$ with $\operatorname{rhs}(X)=u Y v$ where $u, v \in \Sigma^{*}$ and $Y \in V$ two variables $X_{u}, X_{v} \in$ Lo and set $\operatorname{rhs}(X)=X_{u} Y X_{v}, \operatorname{rhs}\left(X_{u}\right)=u$, and $\operatorname{rhs}\left(X_{v}\right)=v$ (if e.g. $u=\varepsilon$, then $X_{u}$ is not present). Moreover, if a symbol $a \in \Sigma$ occurs in a right-hand side of the form $Y^{\omega}, Y^{\bar{\omega}}$, or $\left[Y_{1}, \ldots, Y_{n}\right]^{\eta}$, then we replace that occurrence by a new Lo-variable with right-hand side $a$.

In fact, by this preprocessing all right-hand sides of the form (1) have length at most 3 . This fact will be important when we estimate the size of the final system. From now on variables in Up that have a right-hand side of form (1) or (2) are said to be of type $(1,2)$, all other variables are said to be of type (3-5).

Following [2, proof of Theorem $65 \& 66]$ we will now give an algorithm that produces a proper 2-level system. We will proceed along the hierarchical order of the variables in Up where in each step we possibly add a constant number of new variables and

\footnotetext{
${ }^{5}$ Let, for instance, $\operatorname{rhs}(X)=[Y]^{\eta}$ with $\operatorname{val}(Y)=a[a]^{\eta}$. Then $\operatorname{val}(X)=[a]^{\eta}$ is primitive but $\operatorname{val}(Y)$ is not primitive.
} 
change the right-hand sides of the old variables such that all variables are proper and of the form (1)-(5) and, moreover, all old variables $X$ are of type $(1,2)$ and fulfill the following technical condition (TEC):

(a) If $\operatorname{val}(X)$ has a first block, then $\operatorname{rhs}(X) \in \mathrm{Lo}^{\geq 2} \cup \mathrm{Lo}^{+} \cup \mathrm{pLo}$ * and the first variable of rhs $(X)$ evaluates to the first block of val $(X)$.

(b) If $\operatorname{val}(X)$ has a second block and the first block is scattered, then $\operatorname{rhs}(X) \in$ $\mathrm{Lo}^{\geq 2} \cup \mathrm{Lo}^{\geq 2} \mathrm{UpLo}^{*}$ and the second variable of $\operatorname{rhs}(X)$ evaluates to the second block of $\operatorname{val}(X)$.

(c) If $\operatorname{val}(X)$ has a last block then $\operatorname{rhs}(X) \in \mathrm{Lo}^{\geq 2} \cup \mathrm{Lo}^{*} \mathrm{UpLo}^{+}$and the last variable of $\operatorname{rhs}(X)$ evaluates to the last block of val $(X)$.

(d) If val $(X)$ has a second last block and the last block is scattered, then $\operatorname{rhs}(X) \in$ $\mathrm{Lo}^{\geq 2} \cup \mathrm{Lo}^{*} \cup \mathrm{pLo}{ }^{\geq 2}$ and the second last variable of $\operatorname{rhs}(X)$ evaluates to the second last block of $\operatorname{val}(X)$.

We need the following claim about this property (TEC):

Claim. If $\operatorname{rhs}(X) \in \mathrm{Lo}^{+} \cup \mathrm{Lo}^{*} \cup \mathrm{pLo}{ }^{*}$ and $\operatorname{rhs}(X)$ is good, then $X$ satisfies (TEC).

Proof. By symmetry let us only consider conditions (a) and (b) of (TEC). Assume that $\operatorname{rhs}(X)$ is a good sequence. If $\operatorname{rhs}(X) \in$ Lo*, then Lemma 4.10 implies that the variables in $\operatorname{rhs}(X)$ evaluate to the blocks of $\operatorname{val}(X)$ (recall that $\operatorname{rhs}(X)$ is good). Hence (a) and (b) hold. Next, assume that $\operatorname{rhs}(X) \in \mathrm{Lo}^{\geq 2} \mathrm{UpLo}^{*}$. Again, since $\operatorname{rhs}(X)$ is good, Lemma 4.10 implies that the first two variables in $\operatorname{rhs}(X)$ evaluate to the first two blocks of $\operatorname{val}(X)$. Thus, (a) and (b) hold again. If $\operatorname{rhs}(X) \in \mathrm{UpLo}^{*}$, then the first variable of $\operatorname{rhs}(X)$ evaluates to a non-primitive word. Since $\operatorname{rhs}(X)$ is good, it follows that val $(X)$ does not have a first block and (a) and (b) hold. Finally assume that rhs $(X) \in \operatorname{LoUpLo*}$ and the first two variables of $\operatorname{rhs}(X)$ are $A \in$ Lo and $Z \in \mathrm{Up}$. Then, $\operatorname{val}(A)$ is the first block of $\operatorname{val}(X)$. Since $\operatorname{rhs}(X)$ is good either val $(Z)$ does not have a first block or val $(Z)$ has a first block, $\operatorname{val}(A)$ is uniform, and $(\operatorname{val}(A), \operatorname{val}(Z))$ does not merge. In both cases (a) and (b) are obviously satisfied. This proves the claim.

Actual algorithm. We can now outline our procedure. Consider a variable $X \in \mathrm{Up}$ such that every variables in $\operatorname{rhs}(X)$ is either in Lo or was already processed and is therefore now proper, satisfies (TEC), and is of type $(1,2)$. We need to distinguish on the form of the right-hand side of $X$. In all of the following cases, we reset $\operatorname{rhs}(X)$ either

(i) to a shuffle of variables that are already proper or

(ii) to a good sequence from $\mathrm{Lo}^{+} \cup \mathrm{Lo}^{*} \cup \mathrm{pLo}$ (and all variables in that sequence are already proper).

In (i), $X$ is proper by Definition 4.22(4) (note that $\operatorname{val}(X)$ is not primitive since $X \in$ Up). In (ii) it follows from Lemma 4.10 and Claim 4.6, that $X$ is proper and satisfies (TEC). For every other new upper level variables $Y$ that is introduced, the right-hand side is either 
(i) a non-merging sequence of (already proper) variables or

(ii) $Z^{\omega}$ or $Z^{\bar{\omega}}$, where $Z$ is already proper and $Z Z Z$ does not merge.

In both cases it follows from Definition 4.22 that $Y$ is proper too.

Case 1. $\operatorname{rhs}(X) \in \mathrm{Lo}^{2} \cup \mathrm{Lo}^{3}$ (hence $\operatorname{rhs}(X)$ is semi-good). By applying Lemma 4.24 to rhs $(X)$, we can compute in polynomial time an equivalent good sequence of at most three possibly new Lo-variables (and their corresponding right-hand sides). This sequence becomes the new right-hand side of $X$.

Case 2. $\operatorname{rhs}(X) \in \mathrm{Lo}^{\leq 1} \mathrm{UpLo}^{\leq 1}$. Let $Z$ be the unique Up-variable in $\operatorname{rhs}(X)$. Note that $Z$ is one of the old variables, which has already been processed and hence is proper, of type $(1,2)$, and satisfies (TEC). If $\operatorname{rhs}(Z) \in \mathrm{Lo}^{\geq 2} \cup \mathrm{Lo}^{*} \mathrm{UpLo}^{*}$, then we replace $Z$ in $\operatorname{rhs}(X)$ by $\operatorname{rhs}(Z)$ (if $\operatorname{rhs}(Z)$ is a shuffle, then we leave $Z$ in $\operatorname{rhs}(X)$ ). Recall that $Z$ is proper and satisfies (TEC). It follows easily that the resulting new right-hand side of $X$ is semi-good and in Lo ${ }^{\geq 2} \cup$ Lo $^{*}$ UpLo*. Thus, we can apply Lemma 4.24 and obtain an equivalent good sequence in $\mathrm{Lo}^{+} \cup \mathrm{Lo}^{*} \mathrm{UpLo}^{*}$ (as in Case 1, we will introduce new Lo-variables thereby). This good sequence will be the new right-hand side of $X$.

Case 3. $\operatorname{rhs}(X)=\left[Y_{1}, \ldots, Y_{k}\right]^{\eta}$. Then there is nothing to do. Recall that we assumed that $\operatorname{val}(X)$ is not primitive and hence $X$ is proper and satisfies the technical condition (TEC) as $\operatorname{val}(X)$ neither has a first nor a last block.

Case 4. $\operatorname{rhs}(X)=Y Z$ for some $Y, Z \in$ Up. Here $Y$ and $Z$ are old variables, which have already been processed and therefore are proper, of type $(1,2)$, and satisfy (TEC). If $\operatorname{rhs}(Y) \in \mathrm{Lo}^{\geq 2} \cup \mathrm{Lo}^{*} \mathrm{UpLo}^{*}$ then we replace $Y$ in $Y Z$ by $\operatorname{rhs}(Y)$ (if $\operatorname{rhs}(Y)$ is a shuffle, we leave $Y$ in $Y Z$ ). We proceed analogously with $Z$ in $Y Z$. Since $Y$ and $Z$ are proper and satisfy (TEC), it follows (as in Case 2) that the resulting new right-hand side of $X$ is semi-good and contains at most two variables from Up. Thus we can apply Lemma 4.24 and obtain an equivalent good sequence $u$ of variables with at most two variables from Up (again, we introduce new Lo-variables thereby).

Now, we replace parts in the sequence $u$ in order to get rhs $(X)$. First, assume that $u=A_{1} \cdots A_{k} \in$ Lo $^{+}$. If $k \leq 5$, then $\operatorname{rhs}(X)$ simply becomes $u$ (which is good). If $k \geq 6$, then we introduce a new Up-variable $U$ and set

$$
\operatorname{rhs}(X)=A_{1} A_{2} U A_{k-1} A_{k}, \quad \operatorname{rhs}(U)=A_{3} \cdots A_{k-2} .
$$

Since $u$ is good, both right-hand sides are good as well. Second, assume that $u=$

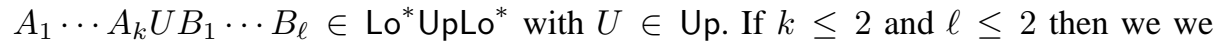
simply set $\operatorname{rhs}(X)=u$. On the other hand, if $k>2$ or $\ell>2$, then we introduce a new Up-variable $V$ and set

$$
\operatorname{rhs}(X)=A_{1} A_{2} V B_{\ell-1} B_{\ell}, \quad \operatorname{rhs}(V)=A_{3} \cdots A_{k} U B_{1} \cdots B_{\ell-2}
$$

(if e.g. $k>2$ but $\ell=1$, then $B_{1} \cdots B_{\ell-2}$ and $B_{\ell-1}$ disappear). Since $u$ is good, $\operatorname{rhs}(X)$ will be good too. Moreover, since $u$ does not merge (by Lemma 4.10), $\operatorname{rhs}(V)$ does not merge as well ( $\operatorname{rhs}(V)$ is not necessarily good). Third, assume that $u=$

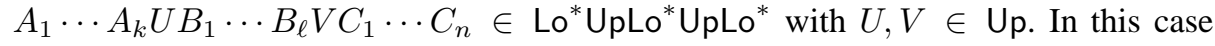
we introduce two new Up-variables $W_{1}$ and $W_{2}$ and set

$\operatorname{rhs}(X)=A_{1} A_{2} W_{1} C_{1} \cdots C_{n}, \operatorname{rhs}\left(W_{1}\right)=W_{2} V, \operatorname{rhs}\left(W_{2}\right)=A_{3} \cdots A_{k} U B_{1} \ldots B_{\ell}$. 
Again, since $u$ is good, $\operatorname{rhs}(X)$ is good as well. Moreover, since $u$ does not merge, neither rhs $\left(W_{1}\right)$ nor $\operatorname{rhs}\left(W_{2}\right)$ merges. Note that the number $n$ in the right-hand side of $X$ above is bounded by $|\operatorname{rhs}(Z)|$. This will be important for estimating the length of right-hands.

Case 5. $\operatorname{rhs}(X)=Y^{\omega}$. Note that $Y$ is either a Lo-variable, or it is an old Up-variable, which has already been processed and hence is proper, of type $(1,2)$, and satisfies (TEC). We can therefore distinguish the following subcases.

Case 5(a). $\operatorname{rhs}(Y)=\left[Z_{1}, \ldots, Z_{n}\right]^{\eta}$ for some $Z_{1}, \ldots, Z_{n} \in$ Lo $\cup$ Up. Then by the general identity $\left(\Gamma^{\eta}\right)^{\omega} \cong \Gamma^{\eta}$ (which follows from Cantor's theorem), we have $\operatorname{val}(X)=$ $\operatorname{val}(Y)$ and we set $\operatorname{rhs}(X)=Y$. Then $X$ is obviously proper. Since we assumed that $\operatorname{val}(X)$ is not primitive $\operatorname{val}(X)$ does not have a first or a last block and (TEC) is satisfied.

Case 5(b). $\operatorname{rhs}(Y) \in$ Lo*UpLo* $^{*}$ Let $\operatorname{rhs}(Y)=u Z v$ with $Z \in$ Up and $u, v \in$ Lo* $^{*}$. Since $Y$ is proper and satisfies (TEC), the infinite sequence $u Z v u Z v \cdots=u(Z v u)^{\omega}$ is semi-good. By applying Lemma 4.24 to the sequence $v u$ of Lo-variables, we obtain an equivalent good sequence $u(Z w)^{\omega}$. Here $w$ is a sequence of (possibly new) Lo-variables such that $w$ represents the irreducible normal form w.r.t. $R$ of the sequence represented by $v u$. Note that $|w| \leq|u v|$. We set

$$
\operatorname{rhs}(X)=u V, \quad \operatorname{rhs}(V)=U^{\omega}, \quad \operatorname{rhs}(U)=Z w .
$$

Since the sequence $u(Z w)^{\omega}$ is good, also the sequence $u V$ is good. Moreover, since $u(Z w)^{\omega}$ does not merge (by Lemma 4.10), the same holds for $\operatorname{rhs}(U)$ and $U U U$ (so $U$ and $V$ are proper by definition).

Case 5(c). $Y \in \operatorname{Lo}$ and hence $\operatorname{val}(Y)$ is primitive. Then the infinite sequence $Y Y Y \cdots$ must be irreducible, because otherwise $\operatorname{val}(Y)$ would be either finite or uniform and $\operatorname{val}(X)=\operatorname{val}\left(Y^{\omega}\right)$ would be primitive. We introduce a new Up-variable $Z$ and set

$$
\operatorname{rhs}(X)=Y Y Z, \quad \operatorname{rhs}(Z)=Y^{\omega} .
$$

Then $\operatorname{rhs}(X)$ is good and $Y Y Y$ does not merge.

Case $5(d)$. $\operatorname{rhs}(Y) \in \mathrm{Lo}^{2}$. Let $\operatorname{rhs}(Y)=A_{1} A_{2}$ for $A_{1}, A_{2} \in$ Lo. Since $Y$ is already proper, we know that $A_{1} A_{2}$ is irreducible. If the infinite sequence $A_{1} A_{2} A_{1} A_{2} \cdots$ is irreducible too, then we introduce a new Up-variables $Z$ and set

$$
\operatorname{rhs}(X)=A_{1} A_{2} Z, \quad \operatorname{rhs}(Z)=Y^{\omega} .
$$

Clearly, rhs $(X)$ is good and $Y Y Y$ does not merge. On the other hand, if $A_{1} A_{2} A_{1} A_{2} \ldots$ is not irreducible, then (since $A_{1} A_{2}$ is irreducible), an $R$-reduction can only occur at a border between $A_{2}$ and $A_{1}$. The case that $\operatorname{val}\left(A_{1}\right)=\operatorname{val}\left(A_{2}\right)=\Gamma^{\eta}$ for some $\Gamma \subseteq \Sigma$ cannot occur (since $A_{1} A_{2}$ is irreducible). If val $\left(A_{2}\right)$ is scattered and right-closed and $\operatorname{val}\left(A_{1}\right)$ is scattered and left-closed, then we introduce a new Lo-variable $B$ and a new Up-variable $Z$ and set

$$
\operatorname{rhs}(X)=A_{1} B Z, \quad \operatorname{rhs}(Z)=B^{\omega}, \quad \operatorname{rhs}(B)=A_{2} A_{1} .
$$


It is straightforward to show that the infinite sequence $A_{1} B B B \cdots$ is irreducible. Hence rhs $(X)$ is good and $B B B$ does not merge. Next, if $\operatorname{val}\left(A_{1}\right)=\Gamma^{\eta}$ and $\operatorname{val}\left(A_{2}\right)=$ $a$ for some $\Gamma \subseteq \Sigma$ and $a \in \Gamma$, then $A_{1} A_{2} A_{1} A_{2} \cdots$ evaluates to $\Gamma^{\eta}$. Hence, $\operatorname{val}(X)$ is primitive, which is a contradiction. Finally, if $\operatorname{val}\left(A_{2}\right)=\Gamma^{\eta}$ and $\operatorname{val}\left(A_{1}\right)=a \in \Gamma$, then $A_{1} A_{2} A_{1} A_{2} \cdots$ evaluates to $a \Gamma^{\eta}=\operatorname{val}(Y)$ and we set $\operatorname{rhs}(X)=Y$.

Case $5(d)$. $\operatorname{val}(Y) \in \mathrm{Lo}^{\geq 3}$. We apply Lemma 4.25 to the irreducible sequence $\operatorname{rhs}(Y)$ and compute sequences $u, v$ of (possibly new) Lo-variables with their corresponding right-hand sides. The infinite sequence $u v^{\omega}$ of Lo-variables is irreducible and evaluates to $\operatorname{val}(Y)$. W.l.o.g. we can assume $|u| \geq 2$ (otherwise, we can replace $u$ by $u v v$ ). We introduce new Up-variables $U$ and $V$ and set

$$
\operatorname{rhs}(X)=u V, \quad \operatorname{rhs}(V)=U^{\omega}, \quad \operatorname{rhs}(U)=v .
$$

(if $|v|=1$, i.e., $v$ consists of a single Lo-variable, then we do not need $U$ ).

Case 6. $\operatorname{rhs}(X)=Y^{\bar{\omega}}$. This case is symmetric to Case 4 .

The resulting system $\mathbb{C}$ is primitive and all Up-variables are proper. On the other hand, $\mathbb{C}$ is not necessarily irredundant. But this can be easily achieved as described in Remark 4.21.

We are now in the position to prove Theorem 4.15.

Proof of Theorem 4.15. It suffices to show that the following problem can be solved in polynomial time:

INPUT: An SES $\mathbb{A}$ and two variables $X, Y$ of $\mathbb{A}$.

QUESTION: $\operatorname{val}(X) \cong \operatorname{val}(Y)$ ?

If both variables $X$ and $Y$ evaluate to primitive words, then we just need to apply Lemma 4.18. If only one of the two evaluates to a primitive word, then $\operatorname{val}(X) \neq$ $\operatorname{val}(Y)$. Hence, we may assume that both $\operatorname{val}(X)$ and $\operatorname{val}(Y)$ are not primitive. In particular, we have $\omega \eta$ - $\operatorname{depth}(X), \omega \eta-\operatorname{depth}(Y)>0$. It is easy to bring $\mathbb{A}$ into the normal form required in Proposition 4.26. Applying Proposition 4.26 to $\mathbb{A}$ gives a proper 2-level system $\mathbb{A}_{0}$. The variables $X$ and $Y$ belong to the upper level part of $\mathbb{A}_{0}$. Starting with $\mathbb{A}_{0}$ we construct a sequence of proper 2-level systems $\mathbb{A}_{j}=\left(\mathrm{Up}_{j}, \mathrm{Lo}_{j}, \mathrm{Lo}_{j-1}, \mathrm{rhs}_{j}\right)$ (with Lo $_{-1}=\Sigma$ ). In order to obtain $\mathbb{A}_{j}$ we apply the procedure of Proposition 4.26 to up $\left(\mathbb{A}_{j-1}\right)$. Let $k$ be maximal such that $X$ and $Y$ belong to the upper level part of $\mathbb{A}_{k}$. Since by Proposition 4.26 in every second step the $\omega \eta$-depth of $X$ and $Y$ strictly decreases we have $k \leq 2 \cdot|\mathbb{A}|$.

Let $0 \leq j \leq k$. By Lemma 4.23 uval $_{j}(X)$ is the lo $\left(\mathbb{A}_{j}\right)$-skeleton of $\operatorname{val}_{j}(X)$ and similarly for $Y$. Hence $\operatorname{val}_{j}(X) \cong \operatorname{val}_{j}(Y)$ if and only if uval $(X) \cong \operatorname{uval}_{j}(Y)$ by Proposition 4.3. Recall that $\mathbb{A}_{j+1}$ is obtained by applying the procedure of Proposition 4.26 to $u p\left(\mathbb{A}_{j}\right)$. We obtain $\operatorname{val}_{j}(X) \cong \operatorname{val}_{j}(Y)$ if and only if $\operatorname{val}_{j+1}(X) \cong$ $\operatorname{val}_{j+1}(Y)$ for all $0 \leq j<k$. Hence, $\operatorname{val}(X) \cong \operatorname{val}(Y)$ if and only if $\operatorname{val}_{k}(X) \cong$ $\operatorname{val}_{k}(Y)$ if and only if uval ${ }_{k}(X) \cong \mathrm{uval}_{k}(Y)$. Now, by the maximality of $k$, uval $_{k}(X)$ or uval ${ }_{k}(Y)$ must be primitive. Hence, using Lemma 4.18, we can check in polynomial time whether uval $k(X) \cong \operatorname{uval}_{k}(Y)$. 
Runtime. Let us analyze the $\operatorname{system} u p\left(\mathbb{A}_{j}\right)$ for $1 \leq j \leq k$. The 2-level system $\mathbb{A}_{j}$ is obtained by applying Proposition 4.26 to up $\left(\mathbb{A}_{j-1}\right)$. Observe that by the construction in the proof, the system up $\left(\mathbb{A}_{j}\right)$ already has the normal form that we require in Proposition 4.26. Let Type(3-5) $)_{j}$ be the set of variables in $U p_{j}$ that are of type (3-5).

Now let us estimate the number $\left|\mathrm{Up}_{j}\right|$ for $1 \leq j \leq k$. Observe that in the proof of Proposition 4.26 in each of the Cases (1)-(3) only new lower level variables are introduced. In each of the Cases (4)-(6) the old variable is turned into a variable of type $(1,2)$ and at most one new variable of type (3-5) is added to $U \mathbf{p}_{j}$. Moreover, additionally at most one new variables of type $(1,2)$ is added to $U \mathrm{p}_{j}$. We conclude that $\mid$ Type(3-5) ${ }_{j}|\leq|$ Type(3-5) $)_{j-1} \mid$ and the total number of variables in $U \mathrm{p}_{j}$ is bounded by $\left|\mathrm{Up}_{j-1}\right|+2 \cdot\left|\operatorname{Type}(3-5)_{j-1}\right|$. Recall that $j \leq k \leq 2|\mathbb{A}|$. Hence $\left|\mathrm{Up}_{j}\right| \leq\left|\mathrm{Up}_{0}\right|+2 j$. $\mid$ Type $(3-5)_{0}|\leq| \mathbb{A}_{0} \mid \cdot(4 \cdot|\mathbb{A}|+1)$ for all $0 \leq j \leq k$.

Let us now estimate the maximal length of a right-hand side in $\mathbb{A}_{j}$. Let us first bound the length of the right-hand side of a variable $X \in U \mathrm{p}_{j} \cap U \mathrm{p}_{j-1}$ (i.e., an old variable). By reanalyzing all cases from the proof of Proposition 4.26, we see that for such a variable $X,\left|\operatorname{rhs}_{j}(X)\right|$ is either at most 5 or it is bounded by $3+\left|\operatorname{rhs}_{j}(Y)\right|$, where $Y \in \mathrm{Up}_{j} \cap \mathrm{Up}_{j-1}$ is an old variable, which was processed before. We therefore obtain $\left|\operatorname{rhs}_{j}(X)\right| \leq 3 \cdot\left|\mathrm{Up}_{j} \cap \mathrm{Up}_{j-1}\right|+5$. Hence, $\left|\mathrm{rhs}_{j}(X)\right| \leq 3 \cdot\left|\mathbb{A}_{0}\right| \cdot(4 \cdot|\mathbb{A}|+1)+5$. For the newly added variables, $X \in \mathrm{Up}_{j} \backslash U \mathrm{vp}_{j-1}$ the size of the right-hand side is bounded by twice the maximal size of a right-hand side of an old variable in $U \mathrm{p}_{j} \cap \mathrm{Up}_{j-1}$ (the factor 2 comes from Case 4). Hence $\mid$ rhs $_{j}(X)|\leq 6 \cdot| \mathbb{A}_{0} \mid \cdot(4 \cdot|\mathbb{A}|+1)+10$ for all $X \in \mathrm{Up}_{j}$. Finally, note that $\left|\mathbb{A}_{0}\right|$ is bounded polynomially bounded in $|\mathbb{A}|$.

Concerning lower level variables of $\mathbb{A}_{j}$, note that the length $\left|\operatorname{rhs}_{j}(A)\right|$ for a lower level variable of $\mathbb{A}_{j}$ is bounded by 2 (if $A$ is introduced in one of the Cases 1-6) or by the maximal length of the right-hand side of a variable from $\mathbb{A}_{j-1}$ (if $A$ is introduced in the preprocessing step). Moreover, in each of the Cases 1-6, the number of new lower level variables that are introduced is bounded by twice the maximal size of a right-hand side of an old variable in $U \mathrm{p}_{j} \cap U \mathrm{p}_{j-1}$ (the factor 2 comes again from Case 4). Hence the number of lower level variables is also bounded polynomially in $|\mathbb{A}|$.

We have shown that the total size of very 2-level system $\mathbb{A}_{j}(1 \leq j \leq k)$ is bounded polynomially in $|\mathbb{A}|$. As the time needed to construct $\mathbb{A}_{j+1}$ from $\mathbb{A}_{j}$ is polynomially bounded by Proposition 4.26, we conclude that the overall running time of our algorithm is polynomially bounded as well.

\subsection{Lower bounds for regular linear orders}

In this section we prove lower bounds for the isomorphism problem for regular words. In fact, all these lower bounds only need a unary alphabet, i.e., they hold for regular linear orders. The results in this section nicely contrast the results from Section 3, where we studied the isomorphism problem for the prefix order trees on regular languages. In this section, we replace the prefix order by the lexicographical order.

Theorem 4.27. The following problem is $\mathrm{P}$-hard (and hence $\mathrm{P}$-complete) for every finite alphabet $\Sigma$ :

INPUT: Two succinct expressions $\mathbb{A}_{1}$ and $\mathbb{A}_{2}$ over the alphabet $\Sigma$. QUESTION: $\operatorname{val}\left(\mathbb{A}_{1}\right) \cong \operatorname{val}\left(\mathbb{A}_{2}\right)$ ? 
Proof. Note that the problem can be solved in polynomial time by Theorem 4.15. Phardness will be shown by a reduction from the monotone circuit value problem. So, let $C$ be a monotone Boolean circuit. We can assume that the gates of $C$ are partitioned into layers $L_{1}, \ldots, L_{n}$, where $L_{1}$ contains all input gates, $L_{n}$ only contains the output gate, and all inputs for a gate from $L_{i+1}$ belong to $L_{i}$. Moreover, $L_{i}(i>1)$ either contains only and-gates or or-gates. We construct an SES $\mathbb{A}$ (over a unary terminal alphabet $\{a\}$ ), which contains for each gate $v$ of $C$ a variable test ${ }_{v}$ and for each layer $d \in\{1, \ldots, n\}$ two variables $\operatorname{good}_{d}$, and $\operatorname{bad}_{d}$ such that the following holds for all gates $v \in L_{d}$ :

(a) Either val $\mathbb{A}_{\mathbb{A}}\left(\right.$ test $\left._{v}\right) \cong \operatorname{val}_{\mathbb{A}}\left(\operatorname{bad}_{d}\right)$ or val $\mathbb{A}_{\mathbb{A}}\left(\right.$ test $\left._{v}\right) \cong \operatorname{val}_{\mathbb{A}}\left(\operatorname{good}_{d}\right)$.

(b) $\operatorname{val}_{\mathbb{A}}\left(\right.$ test $\left._{v}\right) \cong \operatorname{val}_{\mathbb{A}}\left(\operatorname{good}_{d}\right)$ if and only if gate $v$ evaluates to true.

(c) The linear orders $\operatorname{val}_{\mathbb{A}}\left(\operatorname{good}_{d}\right)$ and val $\mathbb{A}_{\mathbb{A}}\left(\operatorname{bad}_{d}\right)$ do not contain an interval isomorphic to $\omega \cdot d$ (recall that $\omega \cdot d$ denotes the linear order $\underbrace{\omega+\cdots+\omega}_{d \text { times }}$ ).

The base case for the first layer is trivial. Set $\operatorname{rhs}_{\mathbb{A}}\left(\operatorname{good}_{1}\right)=a$ and $\operatorname{rhs}_{\mathbb{A}}\left(\operatorname{bad}_{1}\right)=a a$. In other words, $\operatorname{val}_{\mathbb{A}}\left(\operatorname{good}_{1}\right) \cong \mathbf{1}$ and val ${ }_{\mathbb{A}}\left(\operatorname{bad}_{1}\right) \cong \mathbf{2}$. Moreover, rhs $_{\mathbb{A}}\left(\right.$ test $\left._{v}\right)=a$ if $v \in L_{1}$ is a true-gate and rhs $_{\mathbb{A}}\left(\right.$ test $\left._{v}\right)=a a$ if $v \in L_{1}$ is a false-gate.

Now assume that $v \in L_{d+1}$ is a gate with inputs $v_{1}, v_{2} \in L_{d}$. For $n \in \mathbb{N}$ we use the abbreviation

$$
\omega \cdot n=\underbrace{a^{\omega} a^{\omega} \cdots a^{\omega}}_{n \text { times }} .
$$

Moreover, we write $\alpha+\beta$ for the concatenation $\alpha \beta$ of the regular expression $\alpha$ and $\beta$ (which denote regular linear orders since the alphabet is unary). There are two cases:

Case 1. $L_{d+1}$ consists of and-gates. Then we set

$$
\begin{aligned}
\operatorname{rhs}_{\mathbb{A}}\left(\operatorname{test}_{v}\right) & =\left[\omega \cdot d+\operatorname{test}_{v_{1}}, \omega \cdot d+\operatorname{test}_{v_{2}}, \omega \cdot d+\operatorname{good}_{d}\right]^{\eta} \\
\operatorname{rhs}_{\mathbb{A}}\left(\operatorname{good}_{d+1}\right) & =\left[\omega \cdot d+\operatorname{good}_{d}\right]^{\eta} \\
\operatorname{rhs}_{\mathbb{A}}\left(\operatorname{bad}_{d+1}\right) & =\left[\omega \cdot d+\operatorname{good}_{d}, \omega \cdot d+\operatorname{bad}_{d}\right]^{\eta} .
\end{aligned}
$$

Case 2. $L_{d+1}$ consists of or-gates.

$$
\begin{aligned}
\operatorname{rhs}_{\mathbb{A}}\left(\operatorname{test}_{v}\right) & =\left[\omega \cdot d+\operatorname{test}_{v_{1}}, \omega \cdot d+\operatorname{test}_{v_{2}}, \omega \cdot d+\operatorname{bad}_{d}\right]^{\eta} \\
\operatorname{rhs}_{\mathbb{A}}\left(\operatorname{good}_{d+1}\right) & =\left[\omega \cdot d+\operatorname{good}_{d}, \omega \cdot d+\operatorname{bad}_{d}\right]^{\eta} \\
\operatorname{rhs}_{\mathbb{A}}\left(\operatorname{bad}_{d+1}\right) & =\left[\omega \cdot d+\operatorname{bad}_{d}\right]^{\eta} .
\end{aligned}
$$

The above three properties (a), (b), and (c) can be shown by induction on the layer. For layer $L_{1}$ all three properties are trivially true. Now, consider layer $L_{d+1}$. Property (a) follows directly from the induction hypothesis for layer $L_{d}$. Since the linear orders $\operatorname{val}_{\mathbb{A}}\left(\operatorname{good}_{d}\right)$ and val $\mathbb{A}_{\mathbb{A}}\left(\operatorname{bad}_{d}\right)$ are shuffles, (c) holds for layer $L_{d+1}$ too. Finally, for (b) we consider two cases:

Case 1. $v \in L_{d+1}$ is an and-gate. Let $v_{1}, v_{2} \in L_{d}$ be the inputs for $v$. First, assume that $v$ evaluates to true. Then, $v_{1}$ and $v_{2}$ both evaluate to true. Hence, by induction, we get 


$$
\begin{aligned}
& \operatorname{val}_{\mathbb{A}}\left(\operatorname{test}_{v_{1}}\right) \cong \operatorname{val}_{\mathbb{A}}\left(\operatorname{test}_{v_{2}}\right) \cong \operatorname{val}_{\mathbb{A}}\left(\operatorname{good}_{d}\right) \text {. Thus, } \\
& \operatorname{val}_{\mathbb{A}}\left(\operatorname{test}_{v}\right)=\left[\omega \cdot d+\operatorname{val}_{\mathbb{A}}\left(\operatorname{test}_{v_{1}}\right), \omega \cdot d+\operatorname{val}_{\mathbb{A}}\left(\operatorname{test}_{v_{2}}\right), \omega \cdot d+\operatorname{val}_{\mathbb{A}}\left(\operatorname{good}_{d}\right)\right]^{\eta} \\
& \cong\left[\omega \cdot d+\operatorname{val}_{\mathbb{A}}\left(\operatorname{good}_{d}\right)\right]^{\eta} \\
& =\operatorname{val}_{\mathbb{A}}\left(\operatorname{good}_{d+1}\right) \text {. }
\end{aligned}
$$

For the other direction assume that

$$
\begin{aligned}
\operatorname{val}_{\mathbb{A}}\left(\operatorname{test}_{v}\right) & =\left[\omega \cdot d+\operatorname{val}_{\mathbb{A}}\left(\operatorname{test}_{v_{1}}\right), \omega \cdot d+\operatorname{val}_{\mathbb{A}}\left(\operatorname{test}_{v_{2}}\right), \omega \cdot d+\operatorname{val}_{\mathbb{A}}\left(\operatorname{good}_{d}\right)\right]^{\eta} \\
& \cong\left[\omega \cdot d+\operatorname{val}_{\mathbb{A}}\left(\operatorname{good}_{d}\right)\right]^{\eta} .
\end{aligned}
$$

Since neither val $\mathbb{A}_{\mathbb{A}}\left(\operatorname{test}_{v_{1}}\right)$ nor val $\mathbb{A}_{\mathbb{A}}\left(\right.$ test $\left._{v_{2}}\right)$ nor val $\mathbb{A}_{\mathbb{A}}\left(\operatorname{good}_{d}\right)$ contains an interval isomorphic to $\omega \cdot d$, [18, Lemma 23] implies that

$$
\omega \cdot d+\operatorname{val}_{\mathbb{A}}\left(\operatorname{test}_{v_{1}}\right) \cong \omega \cdot d+\operatorname{val}_{\mathbb{A}}\left(\operatorname{test}_{v_{2}}\right) \cong \omega \cdot d+\operatorname{val}_{\mathbb{A}}\left(\operatorname{good}_{d}\right) .
$$

This implies

$$
\operatorname{val}_{\mathbb{A}}\left(\operatorname{test}_{v_{1}}\right) \cong \operatorname{val}_{\mathbb{A}}\left(\operatorname{test}_{v_{2}}\right) \cong \operatorname{val}_{\mathbb{A}}\left(\operatorname{good}_{d}\right) .
$$

Finally, the induction hypothesis yields that both $v_{1}$ and $v_{2}$, and hence also $v$ evaluate to true.

Case 2. $v \in L_{d+1}$ is an or-gate. We can use similar arguments as for Case 1 .

We do not know, whether the lower bound from Theorem 4.27 holds for ordinary expressions too (instead of succinct expressions).

Theorem 4.28. The following problem is $\mathrm{P}$-hard (and hence $\mathrm{P}$-complete):

INPUT: Two DFAs $\mathcal{A}_{1}$ and $\mathcal{A}_{2}$.

QUESTION: $\left(L\left(\mathcal{A}_{1}\right) ; \leq_{\text {lex }}\right) \cong\left(L\left(\mathcal{A}_{2}\right) ; \leq_{\text {lex }}\right)$ ?

Proof. Note that by Theorem 4.1 the problem belongs to P. For P-hardness, it suffices by Theorem 4.27 to construct in logspace from a given succinct expression $\mathbb{A}$ (over a unary terminal alphabet) a DFA $\mathcal{A}$ such that the linear order val( $(\mathbb{A})$ is isomorphic to $\left(L(\mathcal{A}) ; \leq_{\text {lex }}\right)$. But this is accomplished by the construction in the proof of $[29$, Proposition 2].

Theorem 4.1 implies that it can be checked in EXPTIME whether the lexicographical orderings on two regular languages, given by NFAs, are isomorphic. We do not know whether this upper bound is sharp. Currently, we can only prove a lower bound of PSPACE:

Theorem 4.29. The following problem is PSPACE-hard:

INPUT: Two NFAs $\mathcal{A}_{1}$ and $\mathcal{A}_{2}$.

QUESTION: $\left(L\left(\mathcal{A}_{1}\right) ; \leq_{\text {lex }}\right) \cong\left(L\left(\mathcal{A}_{2}\right) ; \leq_{\text {lex }}\right)$ ? 
Proof. We prove PSPACE-hardness by a reduction from the PSPACE-complete problem whether a given NFA $\mathcal{A}$ (over the terminal alphabet $\{a, b\}$ ) accepts $\{a, b\}^{*}[28]$. So let $\mathcal{A}$ be an NFA over the terminal alphabet $\{a, b\}$ and let $K=L(\mathcal{A})$. Let $\Sigma=$ $\left\{0,1, a, b, \$_{1}, \$_{2}\right\}$ and fix the following order on $\Sigma$ :

$$
\$_{1}<0<1<\$_{2}<a<b .
$$

Under this order, $\left(\{0,1\}^{*} 1 ; \leq_{\text {lex }}\right) \cong\left(\{a, b\}^{*} b ; \leq_{\text {lex }}\right) \cong \eta$.

It is straightforward to construct from $\mathcal{A}$ in logspace NFAs for the following languages:

$$
\begin{aligned}
L_{1} & =\{a, b\}^{*} b \$_{1} \\
L_{2} & =K b\{0,1\}^{*} 1 \\
L_{3} & =\{a, b\}^{*} b \$_{2} \\
L & =L_{1} \cup L_{2} \cup L_{3}
\end{aligned}
$$

It follows that

$$
\left(L ; \leq_{\text {lex }}\right) \cong \sum_{w \in\{a, b\}^{*} b} \mathcal{L}(w),
$$

(the sum is taken over all words from $\{a, b\}^{*} b$ in lexicographic order), where

$$
\mathcal{L}(w) \cong \begin{cases}\mathbf{1}+\eta+\mathbf{1} & \text { if } w \in K \\ \mathbf{2} & \text { else. }\end{cases}
$$

Hence, if $K \neq\{a, b\}^{*}$, then $\left(L ; \leq_{\text {lex }}\right)$ contains an interval isomorphic to 2 and therefore is not dense. Hence $\left(L ; \leq_{\text {lex }}\right) \neq \eta$. On the other hand, if $K=\{a, b\}^{*}$, then $\left(L ; \leq_{\text {lex }}\right) \cong$ $(\mathbf{1}+\eta+\mathbf{1}) \cdot \eta \cong \eta$. This proves the theorem.

Remark 4.30. The proof of Theorem 4.29 shows that it is PSPACE-hard to check for a given NFA $\mathcal{A}$, whether $\left(L(\mathcal{A}) ; \leq_{\text {lex }}\right) \cong \eta$. In fact, this problem is PSPACE-complete, since we can check in polynomial space whether $\left(L(\mathcal{A}) ; \leq_{\text {lex }}\right) \cong \eta$ : In polynomial time, we can construct an NFA $\mathcal{B}$ that accepts a convolution of two words ${ }^{6} u \otimes v$ if and only if $u, v \in L(\mathcal{A})$ and there exist words $w_{1}, w_{2}, w_{3} \in L(\mathcal{A})$ such that $w_{1}<_{\text {lex }} u<_{\text {lex }} w_{2}$ and $\left(v \leq_{\text {lex }} u\right.$ or $\left.u<_{\text {lex }} w_{3}<_{\text {lex }} v\right)$. Then, $\left(L(\mathcal{A}) ; \leq_{\text {lex }}\right) \cong \eta$ if and only if $\mathcal{B}$ accepts the set of all convolutions $u \otimes v$ with $u, v \in L(\mathcal{A})$. The latter can be checked in polynomial space.

Remark 4.31. In [9] it is shown that the problem, whether for a given context-free language $L$ the linear order $\left(L ; \leq_{\text {lex }}\right)$ is isomorphic to $\eta$, is undecidable. This result is shown by a reduction from Post's correspondence problem. Note that this result can be also easily deduced using the technique from the above proof: If we start with a pushdown automaton for $\mathcal{A}$ instead of an NFA, then the language $L$ from (6) is context-free. Hence, $\left(L ; \leq_{\text {lex }}\right) \cong \eta$ if and only if $L(\mathcal{A})=\{a, b\}^{*}$. The latter property is a well-known undecidable problem.

\footnotetext{
${ }^{6}$ The convolution of the words $a_{1} a_{2} \cdots a_{m}$ and $b_{1} b_{2} \cdots b_{n}$ is the word $\left(a_{1}, b_{1}\right)\left(a_{2}, b_{2}\right) \cdots\left(a_{k}, b_{k}\right)$, where $k=\max \{m, n\}, a_{i}=\#$ (a dummy symbol) for $m<i \leq k$ and $b_{i}=\#$ for $n<i \leq k$.
} 
In Section 3 we also studied the isomorphism problem for finite trees that are succinctly given by the prefix order on the finite language accepted by a DFA (resp., NFA). To complete the picture, we will finally consider the isomorphism problem for linear orders that consist of a lexicographically ordered finite language, where the latter is represented by a DFA (resp., NFA). Of course, this problem is somehow trivial, since two finite linear orders are isomorphic if and only if they have the same cardinality. Hence, we have to consider the problem whether two given acyclic DFAs (resp. NFAs) accept languages of the same cardinality.

Proposition 4.32. It is $\mathrm{C}_{=} \mathrm{L}$-complete (resp. $\mathrm{C}_{=} \mathrm{P}$-complete) to check whether two given acyclic DFAs (resp., acyclic NFAs) accept languages of the same size.

Proof. The upper bounds are easy: There exists a nondeterministic polynomial time (resp., logspace) machine, which gets an NFA (resp. a DFA) $\mathcal{A}$ over an alphabet $\Sigma$ as input, and has precisely $|L(\mathcal{A})|$ many accepting paths. Let $n$ be the number of states of $n$. The machine first branches nondeterministically for at most $n \cdot \log (|\Sigma|)$ steps and thereby produces a word $w \in \Sigma^{\leq n}$. Then it checks whether $w \in L(\mathcal{A})$ and only accepts it this holds. The checking step can be done in deterministic polynomial time for an NFA and in deterministic logspace for a DFA.

For the lower bound, we first consider the DFA-case. Given two nondeterministic logspace machines $M_{1}, M_{2}$ (over the same input alphabet) together with an input $w$ we can produce in logspace the configuration graphs $G_{1}$ and $G_{2}$ of $M_{1}$ and $M_{2}$, respectively, on input $w$. W.l.o.g. we can assume that $G_{1}$ and $G_{2}$ are acyclic (one can add a step counter to $M_{i}$ ). Now, from $G_{i}$ it is straightforward to construct an acyclic DFA $\mathcal{A}_{i}$ such that $\left|L\left(\mathcal{A}_{i}\right)\right|$ is the number of paths in $G_{i}$ from the initial configuration to the (w.l.o.g. unique) accepting configuration. The latter number is the number of accepting computations of $M_{i}$ on input $w$.

Finally, $\mathrm{C}_{=} \mathrm{P}$-hardness for NFAs follows from [16, Theorem 2.1], where it was shown that counting the number of words accepted by an NFA is \#P-complete.

\subsection{Ordered trees}

Let us briefly discuss the isomorphism problem for ordered regular trees, i.e., regular trees, where the children of a node are linearly ordered. An ordered tree can be viewed as a triple $(A ; \leq, R)$, where $(A ; \leq)$ is a tree as defined in Section 2.3 and the binary relation $R$ is the disjoint union of relations $R_{a}(a \in A)$, where $R_{a}$ is a linear order on the children of $a$. Now, assume that $\mathcal{A}$ is a (deterministic or nondeterministic) finite automaton with input alphabet $\Sigma$ and let $\leq_{\Sigma}$ be a linear order on $\Sigma$. Assume that $\varepsilon \in L(\mathcal{A})$. Then, we can define a finitely branching ordered regular tree $\mathrm{oT}\left(\mathcal{A}, \leq_{\Sigma}\right)$ with $\mathcal{A}$ as follows:

$$
\mathrm{oT}\left(\mathcal{A}, \leq_{\Sigma}\right)=\left(L(\mathcal{A}) ; \leq_{\text {pref }}, \bigcup_{u \in L(\mathcal{A})} R_{u}\right)
$$

where $R_{u}$ is the relation

$$
R_{u}=\left\{(v, w) \mid v, w \text { are children of } u \text { in }\left(L(\mathcal{A}) ; \leq_{\text {pref }}\right), v \leq_{\text {lex }} w\right\} .
$$


This means that we order the children of a node $u \in L(\mathcal{A})$ lexicographically. In the following, we will omit the order $\leq_{\Sigma}$ on the alphabet. The proof of the following result combines ideas from the proof of Theorem 3.1 with Theorem 4.1.

Proposition 4.33. The following problem is $\mathrm{P}$-complete:

INPUT: Two DFAs $\mathcal{A}_{1}$ and $\mathcal{A}_{2}$ with $\varepsilon \in L\left(\mathcal{A}_{1}\right) \cap L\left(\mathcal{A}_{2}\right)$. QUESTION: oT $\left(\mathcal{A}_{1}\right) \cong \mathrm{oT}\left(\mathcal{A}_{2}\right)$ ?

Proof. Similarly to the proof of Theorem 3.1, it suffices to take a DFA $\mathcal{A}=(Q, \Sigma, \delta, F)$ without initial state and two states $p, q \in F$, and to check in polynomial time, whether $\mathrm{o \top}(\mathcal{A}, p) \cong \mathrm{o \top}(\mathcal{A}, q)$, where $\mathrm{oT}(\mathcal{A}, r)=\mathrm{oT}(Q, \Sigma, \delta, r, F)$ for $r \in F$. Define the following equivalence relation on $F$ :

$$
\text { iso }=\{(p, q) \in F \times F \mid \mathrm{oT}(\mathcal{A}, p) \cong \mathrm{oT}(\mathcal{A}, q)\} .
$$

We show that iso can be computed in polynomial time. As in the proof of Theorem 3.1, this will be done with a partition refinement algorithm. We need a few definitions.

Recall from the proof of Theorem 3.1 the definition of the languages $L(\mathcal{A}, p, C)$ and $K(\mathcal{A}, p, C) \subseteq L(\mathcal{A}, p, C)$ for $p \in F$ and $C \subseteq F$. Assume that $R$ is an equivalence relation on $F$ and let $m$ be the number of equivalence classes of $R$. Fix an arbitrary bijection $f$ between the the alphabet $\{1, \ldots, m\}$ and the set of equivalence classes of $R$. With $R$ and $p \in F$ we associate a partitioned DFA $\mathcal{A}(p, R)$ as follows: Take the DFA for the language $L(\mathcal{A}, p, F)$ as defined in the proof of Theorem 3.1 and set $F_{i}=f(i)$ $(1 \leq i \leq m)$, which is the set of final states associated with symbol $i$. Finally, define the regular word $w(p, R)=w(\mathcal{A}(p, R))$ over the alphabet $\{1, \ldots, m\}$. We define the new equivalence relation $\widetilde{R}$ on $F$ as follows:

$$
\widetilde{R}=\{(p, q) \in R \mid w(p, R) \cong w(q, R)\} .
$$

Thus, $\widetilde{R}$ is a refinement of $R$ which, by Theorem 4.1, can be computed in polynomial time from $R$. Let us define a sequence of equivalence relations $R_{0}, R_{1}, \ldots$ on $F$ as follows: $R_{0}=F \times F, R_{i+1}=\widetilde{R}_{i}$. Then, there exists $k<|F|$ such that $R_{k}=R_{k+1}$. We claim that $R_{k}=$ iso.

For the inclusion iso $\subseteq R_{k}$, one shows, by induction on $i$, that iso $\subseteq R_{i}$ for all $1 \leq i \leq k$. The point is that for every equivalence relation $R$ on $F$ with iso $\subseteq R$, we also have iso $\subseteq \widetilde{R}$. To see this, assume that iso $\subseteq R$ but there is $(p, q) \in$ iso, which does not belong to $\widetilde{R}$. Since $(p, q)$ belongs to $R$, we must have $w(p, R) ¥ w(q, R)$. On the other hand, since $(p, q) \in$ iso, it follows that the regular words $w(p$, iso $)$ and $w(q$, iso $)$ are isomorphic. But since iso $\subseteq R, w(p, R)$ is a homomorphic image of $w(p$, iso $)$ and similarly for $w(q, R)$. Thus, also $w(p, R)$ and $w(q, R)$ are isomorphic, which is a contradiction.

For the inclusion $R_{k} \subseteq$ iso, we show that if $R$ is an equivalence relation on $F$ such that $R=\widetilde{R}$ (this holds for $R_{k}$ ), then $R \subseteq$ iso. For this, take a pair $\left(p_{1}, p_{2}\right) \in R$. Take the tree $\mathrm{oT}\left(\mathcal{A}, p_{i}\right)$. We assign types in form of final states to the nodes of oT $\left(\mathcal{A}, p_{i}\right)$ in the same way as in the proof of Theorem 3.1. We now construct an isomorphism $f: \mathrm{oT}\left(\mathcal{A}, p_{1}\right) \rightarrow \mathrm{oT}\left(\mathcal{A}, p_{2}\right)$ as the limit of isomorphisms $f_{n}, n \geq 1$. Here, $f_{n}$ is an 
isomorphism between the trees that result from oT $\left(\mathcal{A}, p_{1}\right)$ and o $\mathrm{T}\left(\mathcal{A}, p_{2}\right)$ by cutting off all nodes below level $n$. Let us call these trees $\left.\mathrm{OT}\left(\mathcal{A}, p_{i}\right)\right|_{n}(i \in\{1,2\})$. Moreover, if an $f_{n}$ maps a node $u_{1}$ of type $q_{1}$ to a node $u_{2}$ of type $q_{2}$, then we will have $\left(q_{1}, q_{2}\right) \in R$. Assume that $f_{n}$ is already constructed and let $u_{1}$ of type $q_{1}$ be a leaf of $\mathrm{OT}\left(\mathcal{A}, p_{1}\right) \Upsilon_{n}$. Let $u_{2}=f\left(u_{1}\right)$ be of type $q_{2}$. Then we have $\left(q_{1}, q_{2}\right) \in R$ and hence the regular words $w\left(q_{1}, R\right)$ and $w\left(q_{2}, R\right)$ are isomorphic. Let $g$ be an isomorphism. The elements of these regular words correspond to the children of $u_{1}$ and $u_{2}$, respectively. More precisely, if $v_{i}$ belongs to the domain of $w\left(q_{i}, R\right)$, then $u_{i} v_{i}$ is a child of $u_{i}$ and vice versa. Clearly, $g$ can be also viewed as an isomorphism between the lexicographical orderings on the children of $u_{1}$ and $u_{2}$, respectively. Moreover, by definition of the regular words $w\left(q_{1}, R\right)$ and $w\left(q_{2}, R\right)$, if $g$ maps some $u_{1} v_{1}$ of type $r_{1}$ to $u_{2} v_{2}$ of type $r_{2}$, then $\left(r_{1}, r_{2}\right) \in R$. By choosing such an isomorphism $g$ for every pair $\left(u_{1}, f\left(u_{1}\right)\right)$ of leaves in $\left.\mathrm{\circ T}\left(\mathcal{A}, p_{1}\right)\right|_{n}$ and $\left.\mathrm{\circ T}\left(\mathcal{A}, p_{2}\right)\right|_{n}$, respectively, we can extend $f_{n}$ to $f_{n+1}$.

Let us now consider prefix-closed automata. Here, we can improve the upper bound from Theorem 4.33 to NL.

Proposition 4.34. The following problem is NL-complete:

INPUT: Two prefix-closed DFAs $\mathcal{A}_{1}$ and $\mathcal{A}_{2}$. QUESTION: OT $\left(\mathcal{A}_{1}\right) \cong \mathrm{oT}\left(\mathcal{A}_{2}\right)$ ?

Proof. Again, it suffices to take a prefix-closed DFA $\mathcal{A}=(Q, \Sigma, \delta, Q)$ without initial state, and two states $p, q \in Q$, and two check in NL, whether oT $(Q, \Sigma, \delta, p, Q) \cong$ oT $(Q, \Sigma, \delta, p, Q)$. By the complement closure of $\mathrm{NL}$, it suffices to check nondeterministically in logarithmic space, whether $\mathrm{oT}(Q, \Sigma, \delta, p, Q) \neq \mathrm{o} \mathrm{T}(Q, \Sigma, \delta, p, Q)$ This can be done as follows: Let $a_{1}<a_{2} \cdots<a_{m}$ and $b_{1}<b_{2}<\cdots<b_{n}$ the transition labels of the outgoing transitions of $p$ and $q$, respectively. If $m \neq n$ then clearly $\mathrm{\circ} \mathrm{T}(Q, \Sigma, \delta, p, Q) \neq \mathrm{o} \mathrm{T}(Q, \Sigma, \delta, q, Q)$ and the algorithm can accept. If $n=m$, then $\mathrm{\circ} \mathrm{T}(Q, \Sigma, \delta, p, Q) \neq \mathrm{\circ} \mathrm{\top}(Q, \Sigma, \delta, q, Q)$ if and only if there exists $1 \leq i \leq m$ such that $\mathrm{\circ} \mathrm{T}\left(Q, \Sigma, \delta, \delta\left(p, a_{i}\right), Q\right) \neq \mathrm{\circ} \mathrm{\top}\left(Q, \Sigma, \delta, \delta\left(q, b_{i}\right), Q\right)$. Hence, the algorithm will simply guess $1 \leq i \leq m$ and replace the state pair $(p, q)$ by $\left(\delta\left(p, a_{i}\right), \delta\left(q, b_{i}\right)\right)$. In this way, the algorithm only has to store two states of $\mathcal{A}$, which is possible in logspace.

NL-hardness can be shown by a reduction from the complement of the graph accessibility problem. Take a directed graph $G=(V, E)$ and two nodes $s, t \in V$. Add to each node of $V$ loops, so that every node $v \in V \backslash\{t\}$ has outdegree $n$ (where $n$ can be taken as the maximal outdegree of a node of $G$ ) and $t$ has outdegree $n+1$. Then label the edges of the resulting multigraph arbitrarily by symbols so that we obtain a DFA $\mathcal{A}$ (the initial state is $s$ and all states are final). Then there is no path from $s$ to $t$ in $G$ if and only if the tree $\mathrm{oT}(\mathcal{A})$ is a full $n$-ary tree.

Corollary 4.35. The following problem is PSPACE-complete:

INPUT: Two prefix-closed NFAs $\mathcal{A}_{1}$ and $\mathcal{A}_{2}$.

QUESTION: OT $\left(\mathcal{A}_{1}\right) \cong \mathrm{oT}\left(\mathcal{A}_{2}\right)$ ?

Proof. The PSPACE upper bound follows from Proposition 4.34, using Lemma 2.1 and the obvious fact that the power set automaton of a given NFA can be produced by 


\begin{tabular}{c|c|c} 
& DFA & NFA \\
\hline acyclic & \multirow{2}{*}{ P-complete } & PSPACE-complete \\
arbitrary & & EXPTIME-complete \\
\hline
\end{tabular}

Table 1. Main results for the isomorphism problem for regular trees

\begin{tabular}{|c|c|c|}
\hline & DFA & NFA \\
\hline acyclic & $\mathrm{C}=\mathrm{L}$-complete & $\mathrm{C}=\mathrm{P}$-complete \\
\hline arbitrary & P-complete & $\begin{array}{l}\text { PSPACE-hard, } \\
\text { in EXPTIME }\end{array}$ \\
\hline
\end{tabular}

Table 2. Main results for the isomorphism problem for regular linear orders

a PSPACE-transducer. For the PSPACE lower bound, note that for an NFA $\mathcal{A}$ over an alphabet $\Sigma$ we have $L(\mathcal{A})=\Sigma^{*}$ if and only if $\mathrm{oT}(\mathcal{A})$ is a full $|\Sigma|$-ary tree. But universality for NFAs is PSPACE-complete [28].

\section{Conclusion and open problems}

Table 1 (Table 2) summarizes our complexity results for the isomorphism problem for regular trees (regular linear orders). Let us conclude with some open problems. As can be seen from Table 2, there is a complexity gap for the isomorphism problem for regular linear orders that are represented by NFAs. This problem belongs to EXPTIME and is PSPACE-hard. Another interesting problem concerns the equivalence problem for straight-line programs (i.e., succinct expressions that generate finite words, or equivalently, acyclic partitioned DFAs, or equivalently, context-free grammars that generate a single word). Plandowski has shown that this problem can be solved in polynomial time. Recall that this result is fundamental for our polynomial time algorithm for succinct expressions (Theorem 4.15). In [10], it was conjectured that the equivalence problem for straight-line programs is $\mathrm{P}$-complete, but this is still open.

\section{References}

1. V. Bárány, E. Grädel, and S.Rubin. Automata-based presentations of infinite structures. In Finite and Algorithmic Model Theory, number 379 in London Mathematical Society Lecture Notes Series. Cambridge University Press, 2011. to appear.

2. Stephen L. Bloom and Zoltán Ésik. The equational theory of regular words. Information and Computation, 197(1-2):55-89, 2005.

3. Stephen L. Bloom and Zoltán Ésik. Algebraic linear orderings. Technical report, arXiv.org, 2010. http://arxiv.org/abs/1002.1624.

4. Ronald V. Book and Friedrich Otto. String-Rewriting Systems. Springer, 1993. 
5. Ashok K. Chandra, Dexter C. Kozen, and Larry J. Stockmeyer. Alternation. Journal of the Association for Computing Machinery, 28(1):114-133, 1981.

6. Bruno Courcelle. Frontiers of infinite trees. ITA, 12(4), 1978.

7. Bruno Courcelle. The definability of equational graphs in monadic second-order logic. In Proceedings of the 16th International Colloquium on Automata, Languages and Programming (ICALP 1989), number 372 in Lecture Notes in Computer Science, pages 207-221. Springer, 1989.

8. Zoltán Ésik. Representing small ordinals by finite automata. In Proceedings ofthe Twelfth Annual Workshop on Descriptional Complexity of Formal Systems, DCFS 2010, volume 31 of EPTCS, pages 78-87, 2010.

9. Zoltán Ésik. An undecidable property of context-free linear orders. Information Processing Letters, 111(3):107-109, 2011.

10. Leszek Gasieniec, Alan Gibbons, and Wojciech Rytter. Efficiency of fast parallel pattern searching in highly compressed texts. In M. Kutylowski, L. Pacholski, and T. Wierzbicki, editors, Proceedings of the 24th International Symposium on Mathematical Foundations of Computer Science (MFCS'99), Szklarska Poreba (Poland), number 1672 in Lecture Notes in Computer Science, pages 48-58. Springer, 1999.

11. Leszek Gasieniec, Marek Karpinski, Wojciech Plandowski, and Wojciech Rytter. Efficient algorithms for Lempel-Ziv encoding (extended abstract). In Rolf G. Karlsson and Andrzej Lingas, editors, Proceedings of the 5th Scandinavian Workshop on Algorithm Theory (SWAT 1996), Reykjavík (Iceland), number 1097 in Lecture Notes in Computer Science, pages 392403. Springer, 1996.

12. Leslie M. Goldschlager. The monotone and planar circuit value problems are log space complete for P. SIGACT News, 9(2):25-99, 1977.

13. Stephan Heilbrunner. An algorithm for the solution of fixed-point equations for infinite words. ITA, 14(2):131-141, 1980.

14. Birgit Jenner, Johannes Köbler, Pierre McKenzie, and Jacobo Torán. Completeness results for graph isomorphism. Journal of Computer and System Sciences, 66(3):549-566, 2003.

15. Paris C. Kanellakis and Scott A. Smolka. CCS expressions, finite state processes, and three problems of equivalence. Information and Computation, 86(1), 1990.

16. Sampath Kannan, Z. Sweedyk, and Stephen R. Mahaney. Counting and random generation of strings in regular languages. In Proceedings of SODA'95, pages 551-557, 1995.

17. Bakhadyr Khoussainov, André Nies, Sasha Rubin, and Frank Stephan. Automatic structures: richness and limitations. Logical Methods in Computer Science, 3(2):2:2, 18 pp. (electronic), 2007.

18. Dietrich Kuske, Jiamou Liu, and Markus Lohrey. The isomorphism problem on classes of automatic structures. Technical report, arXiv.org, 2010. http://arxiv.org/abs / 1001.2086 .

19. Dietrich Kuske, Jiamou Liu, and Markus Lohrey. The isomorphism problem on classes of automatic structures with transitive relations. submitted for publication, extended version of a paper presented at LICS 2010, 2011.

20. Yury Lifshits. Processing compressed texts: A tractability border. In Bin Ma and Kaizhong Zhang, editors, Proceedings of the 18th Annual Symposium on Combinatorial Pattern Matching (CPM 2007), London (Canada), number 4580 in Lecture Notes in Computer Science, pages 228-240. Springer, 2007.

21. Steven Lindell. A logspace algorithm for tree canonization (extended abstract). In Proceedings of STOC'92, pages 400-404. ACM Press, 1992.

22. Masamichi Miyazaki, Ayumi Shinohara, and Masayuki Takeda. An improved pattern matching algorithm for strings in terms of straight-line programs. In Alberto Apostolico and Jotun Hein, editors, Proceedings of the 8th Annual Symposium on Combinatorial Pattern Matching 
(CPM 97), Aarhus (Denmark), number 1264 in Lecture Notes in Computer Science, pages 1-11. Springer, 1997.

23. C. H. Papadimitriou. Computational Complexity. Addison Wesley, 1994.

24. Wojciech Plandowski. Testing equivalence of morphisms on context-free languages. In Jan van Leeuwen, editor, Second Annual European Symposium on Algorithms (ESA'94), Utrecht (The Netherlands), number 855 in Lecture Notes in Computer Science, pages 460470. Springer, 1994.

25. Wojciech Plandowski and Wojciech Rytter. Complexity of language recognition problems for compressed words. In Juhani Karhumäki, Hermann A. Maurer, Gheorghe Paun, and Grzegorz Rozenberg, editors, Jewels are Forever, Contributions on Theoretical Computer Science in Honor of Arto Salomaa, pages 262-272. Springer, 1999.

26. J. Rosenstein. Linear Ordering. Academic Press, 1982.

27. Wojciech Rytter. Grammar compression, LZ-encodings, and string algorithms with implicit input. In J. Diaz, J. Karhumäki, A. Lepistö, and D. Sannella, editors, Proceedings of the 31st International Colloquium on Automata, Languages and Programming (ICALP 2004), Turku (Finland), number 3142 in Lecture Notes in Computer Science, pages 15-27. Springer, 2004.

28. Larry J. Stockmeyer and A. R. Meyer. Word problems requiring exponential time (preliminary report). In Proceedings of the 5th Annual ACM Symposium on Theory of Computing (STOCS 73), pages 1-9. ACM Press, 1973.

29. Wolfgang Thomas. On frontiers of regular trees. ITA, 20(4):371-381, 1986. 